Supplementary Information

\title{
Stereocontrolled Sequence-Defined Oligotriazoles through Metal-Free Elongation Strategies
}

\author{
Xiaojun Wang, Xueyan Zhang, Yunxin Sun, and Shengtao Ding* \\ State Key Laboratory of Organic-Inorganic Composites, College of Chemical Engineering, Beijing University \\ of Chemical Technology, Beijing 100029, China.
}

\section{Table of Contents}

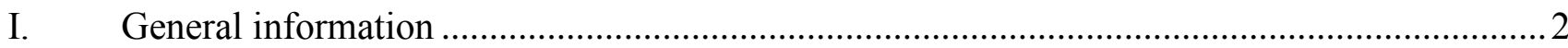

II. Preparation of chiral triazole monomers ....................................................................... 3

III. Enantiomeric excess investigations on $N$-Boc- $(L)$-prolinol, 2 and triazole monomers $\ldots \ldots \ldots . . .7$

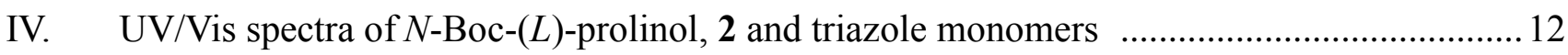

V. Synthesis and characterization of sequence-defined oligotriazoles .................................... 15

VI. Possible mechanisms for MS/MS fragmentation ...................................................... 25

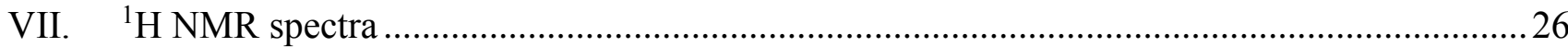

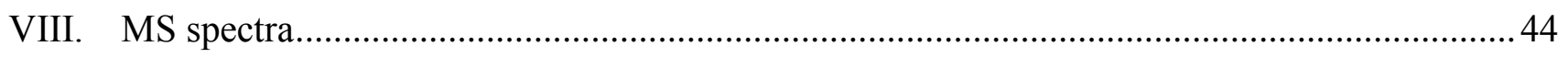




\section{General information}

All air or moisture sensitive reactions were conducted in oven-dried glassware under nitrogen atmosphere using dry solvents. Flash column chromatography was performed over silica gel (200-300 mesh) purchased from Qingdao Puke Co., China. Alkyne and common organic chemicals were purchased from commercial suppliers, such as Sigma-Aldrich ${ }^{\circledR}$ and $\mathrm{J}_{\mathrm{K}} \mathrm{K}^{\circledR}$ Scientific Ltd., and used as received. Iridium complexes were purchased from Strem ${ }^{\circledR}$ Chemicals, Inc.

NMR. ${ }^{1} \mathrm{H}$ and ${ }^{13} \mathrm{C}$ spectrum were collected on a Bruker AV $400 \mathrm{MHz}$ NMR spectrometer using residue solvent peaks as an internal standard $\left({ }^{1} \mathrm{H} \mathrm{NMR}: \mathrm{CDCl}_{3}\right.$ at $7.26 \mathrm{ppm},{ }^{13} \mathrm{C} \mathrm{NMR}: \mathrm{CDCl}_{3}$ at $\left.77.0 \mathrm{ppm}\right)$. MS. ESI-MS experiments was measured using Agilent 6540 UHD Accurate-Mass Q-TOF LC/MS. MALDI-TOF-MS was measured on a AB Sciex 5800 MALDI-TOF/TOF mass spectrometer using $\alpha$ cyano-4-hydroxycinnamic acid (CHCA) or 2,5-dihydroxybenzoic acid (DHB) as the matrix.

MS/MS. MS/MS spectra were acquired using collision-induced dissociation fragmentation. The MALDI-TOF-MS/MS experiments were conducted on a AB Sciex 5800 MALDI-TOF/TOF. The sample was dissolved in $\mathrm{MeOH}$ containing $10 \mathrm{mM} \mathrm{NH}_{4} \mathrm{OAc}$.

SEC. Sequence-defined oligomer samples $(5 \mathrm{mg})$ were dissolved in THF (1 mL) and filtered prior to injection. SEC analyses were performed on a Waters 1525 Gel chromatography with three mixed-bed GPC columns in series (three Waters Styragel HT3 THF (7.8*300mm Column)), and THF mobile phase run at $35^{\circ} \mathrm{C}$ for $40 \mathrm{~min}$. The differential refractive index of each compound was monitored using a WAT038040 (2414) detector.

UV. UV/Vis spectroscopy was performed on a Metash UV-6000PC spectrometer. The spectra were recorded between 200 and $600 \mathrm{~nm}$, with a bandwidth of $2.0 \mathrm{~nm}$, time per point $1 \mathrm{~s}$ and two repetitions. Concentration: $2.5 \times 10^{-4} \mathrm{M}$ in methanol.

CD. CD spectroscopy was performed on a Jasco J-815 CD spectrometer. Solution samples were measured using a $1 \mathrm{~mm}$ cuvette. Sequence-defined oligomer samples $(1.2 \mathrm{mg})$ were dissolved in $\mathrm{MeOH}(3 \mathrm{~mL})$.

Thermogravimetric analysis (TGA). TGA were performed on a thermogravimetric analyzer (Mettler TGA/DSC3+) at a heating rate of $10{ }^{\circ} \mathrm{C} / \mathrm{min}$ from $30{ }^{\circ} \mathrm{C}$ to $800{ }^{\circ} \mathrm{C}$ under the nitrogen atmosphere.

Theoretical monoisotopic mass. All of the theoretical $\mathrm{m} / \mathrm{z}$ values are monoisotopic mass values calculated by using ChemBioOffice 2010 . 


\section{Preparation of chiral triazole monomers}

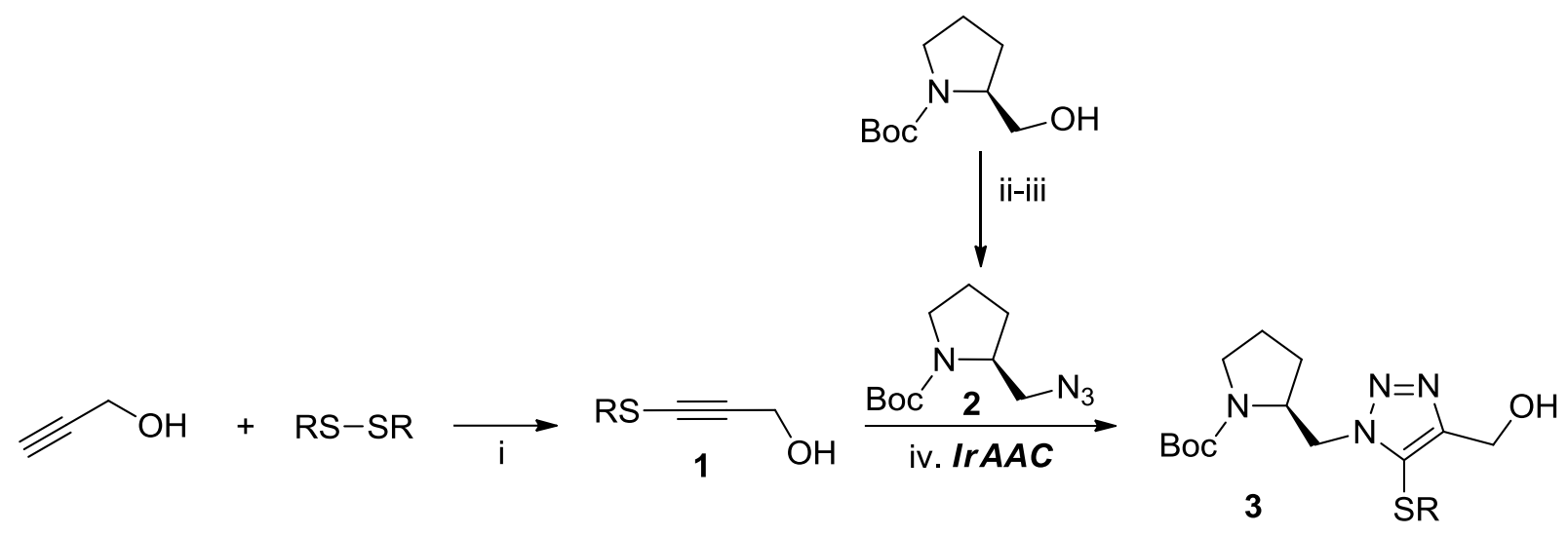

Scheme S1. Preparation of chiral triazole monomers.

\section{General procedures}

i. At $-78{ }^{\circ} \mathrm{C}$, to the solution of prop-2-yn-1-ol (1.0 eq.) in THF (0.25 M) under $\mathrm{N}_{2}$ atmosphere was slowly added $n$-BuLi (2.0 eq.). The reaction mixture was stirred at the same temperature for $1 \mathrm{~h}$ before disulfide (1.0 eq.) and iodoethane (1.0 eq.) were added. Then the reaction mixture was allowed to warm to room temperature and stirred for $2 \mathrm{~h}$ before a saturated aqueous $\mathrm{NH}_{4} \mathrm{Cl}$ solution was added. The aqueous phase was separated and extracted with ethyl acetate for three times. The combined organic phase was washed with brine, dried over $\mathrm{Na}_{2} \mathrm{SO}_{4}$, and evaporated under vacuum to give the crude product of thioalkyne 1, which was then purified by silica gel column chromatography.

ii. $N$-Boc- $(L)$-Prolinol was dissolved in DCM $(0.5 \mathrm{M})$ with subsequent addition of $\mathrm{Et}_{3} \mathrm{~N}(2.0$ eq.). Then the solution of methanesulfonyl chloride ( $\mathrm{MsCl}, 1.5$ eq.) in DCM (1.0 M) was slowly added into the previous mixture. The reaction mixture was stirred at room temperature until the reaction completed (confirmed by TLC), and then washed with brine (three times), dried over $\mathrm{Na}_{2} \mathrm{SO}_{4}$, filtered, and evaporated under vacuum to give the residue of prolinol involving OMs group.

iii. The building unit involving OMs group (1.0 eq.) and $\mathrm{NaN}_{3}$ (1.5 eq.) were added to DMF. The reaction mixture was then heated to $80{ }^{\circ} \mathrm{C}$ for $2-5 \mathrm{~h}$ until the reaction completed, which was confirmed by TLC. The solution was then cooled to room temperature, diluted with ethyl acetate, washed with brine (three times), dried over $\mathrm{Na}_{2} \mathrm{SO}_{4}$, filtered, and evaporated under vacuum. The crude product was purified by column chromatography on silica gel to afford the related pure chiral azide 2.

iv. In a glove box, to an oven-dried vial was added the chiral azide 2 (1.2 eq.), thioalkyne monomer (1.0 eq.), $[\operatorname{Ir}(\mathrm{COD}) \mathrm{Cl}]_{2}(2 \mathrm{~mol} \%)$ and DCE $(0.5 \mathrm{M})$. The vial was capped and removed from the glove box. The reaction mixture was stirred at room temperature for $2-6 \mathrm{~h}$ until the reaction 
completed (confirmed by TLC), and then concentrated under reduced pressure. The residue was purified by silica gel flash column chromatography to give the desired product.

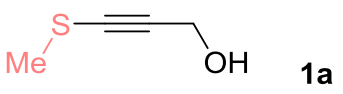

3-(Methylthio)prop-2-yn-1-ol (1a)

$\mathrm{Rf}=0.5(\mathrm{PE} / \mathrm{EA}=5: 1)$.

${ }^{1} \mathbf{H}$ NMR $\left(400 \mathrm{MHz}, \mathrm{CDCl}_{3}\right) \delta 4.32(\mathrm{~s}, 2 \mathrm{H}), 2.36(\mathrm{~s}, 3 \mathrm{H})$.

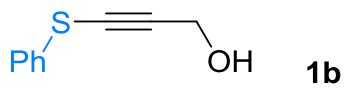

3-(Phenylthio)prop-2-yn-1-ol (1b)

$\mathrm{Rf}=0.5(\mathrm{PE} / \mathrm{EA}=5: 1)$.

${ }^{1} \mathbf{H}$ NMR $\left(400 \mathrm{MHz}, \mathrm{CDCl}_{3}\right) \delta 7.43(\mathrm{~d}, J=8.0 \mathrm{~Hz}, 2 \mathrm{H}), 7.36-7.32(\mathrm{~m}, 2 \mathrm{H}), 7.25-7.19(\mathrm{~m}, 1 \mathrm{H})$, $4.48(\mathrm{~d}, J=8.0 \mathrm{~Hz}, 2 \mathrm{H})$.

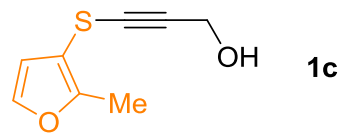

\section{3-((2-Methylfuran-3-yl)thio)prop-2-yn-1-ol (1c)}

$\mathrm{Rf}=0.5(\mathrm{PE} / \mathrm{EA}=5: 1)$.

${ }^{1} \mathbf{H}$ NMR $\left(400 \mathrm{MHz}, \mathrm{CDCl}_{3}\right) \delta 7.28(\mathrm{~s}, 1 \mathrm{H}), 6.44(\mathrm{~s}, 1 \mathrm{H}), 4.33(\mathrm{~s}, 2 \mathrm{H}), 2.35(\mathrm{~s}, 3 \mathrm{H})$.

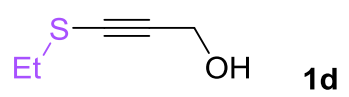

3-(Ethylthio)prop-2-yn-1-ol (1d)

$\mathrm{Rf}=0.5(\mathrm{PE} / \mathrm{EA}=5: 1)$.

${ }^{1} \mathbf{H} \operatorname{NMR}\left(400 \mathrm{MHz}, \mathrm{CDCl}_{3}\right) \delta 4.38(\mathrm{~s}, 2 \mathrm{H}), 2.74(\mathrm{q}, J=8.0 \mathrm{~Hz}, 2 \mathrm{H}), 1.40(\mathrm{t}, J=8.0 \mathrm{~Hz}, 3 \mathrm{H})$.

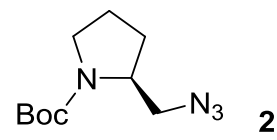

tert-Butyl (L)-2-(azidomethyl)pyrrolidine-1-carboxylate (2)

$\mathrm{Rf}=0.6(\mathrm{PE} / \mathrm{EA}=5: 1)$.

${ }^{1} \mathbf{H}$ NMR $\left(400 \mathrm{MHz}, \mathrm{CDCl}_{3}\right) \delta$ 4.00-3.78 (m, $\left.1 \mathrm{H}\right), 3.63-3.24(\mathrm{~m}, 4 \mathrm{H}), 2.04-1.76(\mathrm{~m}, 4 \mathrm{H}), 1.46(\mathrm{~s}, 9$ $\mathrm{H})$. 


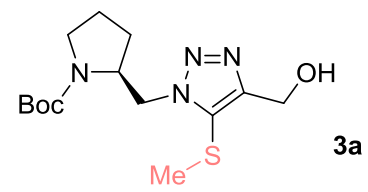

tert-Butyl (L)-2-((4-(hydroxymethyl)-5-(methylthio)-1H-1,2,3-triazol-1-yl)methyl)pyrrolidine-1carboxylate (3a)

$\mathrm{Rf}=0.5(\mathrm{DCM} / \mathrm{MeOH}=20: 1)$.

${ }^{1} \mathbf{H}$ NMR (400 MHz, $\left.\mathrm{CDCl}_{3}\right) \delta 4.77$ (d, $\left.J=4.0 \mathrm{~Hz}, 2 \mathrm{H}\right), 4.64-4.29$ (m, $\left.2 \mathrm{H}\right), 4.26-4.19$ (m, $\left.1 \mathrm{H}\right)$, 3.69-3.61 (m, $1 \mathrm{H}), 3.43-3.13$ (m, $2 \mathrm{H}), 2.36$ (s, $3 \mathrm{H}), 1.90-1.52$ (m, $4 \mathrm{H}), 1.42$ (s, $9 \mathrm{H})$.

ESI-MS: $m / z$ calcd. for $\mathrm{C}_{14} \mathrm{H}_{25} \mathrm{~N}_{4} \mathrm{O}_{3} \mathrm{~S}(\mathrm{M}+\mathrm{H})^{+} 329.1642$, found 329.1656 .

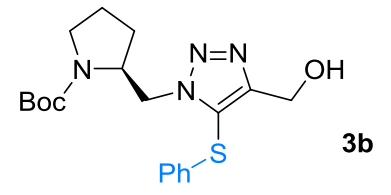

tert-Butyl (L)-2-((4-(hydroxymethyl)-5-(phenylthio)-1H-1,2,3-triazol-1-yl)methyl)pyrrolidine-1carboxylate (3b)

$\mathrm{Rf}=0.5(\mathrm{DCM} / \mathrm{MeOH}=20: 1)$.

${ }^{1}$ H NMR (400 MHz, $\left.\mathrm{CDCl}_{3}\right) \delta$ 7.35-7.15 (m, $\left.4 \mathrm{H}\right)$, 7.06-7.03 (m, $\left.1 \mathrm{H}\right), 4.75$ (s, $\left.2 \mathrm{H}\right), 4.57-4.22$ (m, 3 H), 3.55-3.14 (m, 3 H), 1.80-1.55 (m, 4 H), 1.7-1.41 (m, 9 H).

ESI-MS: $m / z$ calcd. for $\mathrm{C}_{19} \mathrm{H}_{27} \mathrm{~N}_{4} \mathrm{O}_{3} \mathrm{~S}(\mathrm{M}+\mathrm{H})^{+} 391.1798$, found 391.1891 .

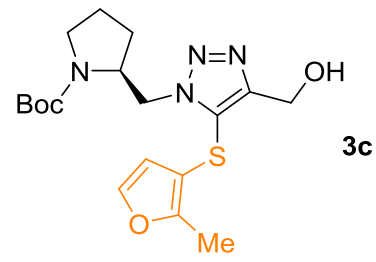

tert-Butyl (L)-2-((4-(hydroxymethyl)-5-((2-methylfuran-3-yl)thio)-1H-1,2,3-triazol-1yl)methyl)pyrrolidine-1-carboxylate (3c)

$\mathrm{Rf}=0.5(\mathrm{DCM} / \mathrm{MeOH}=20: 1)$.

${ }^{1} \mathbf{H}$ NMR (400 MHz, $\left.\mathrm{CDCl}_{3}\right) \delta$ 7.30-7.19 (m, $\left.1 \mathrm{H}\right), 6.37-6.20$ (m, $\left.1 \mathrm{H}\right), 4.74-4.69$ (m, $\left.2 \mathrm{H}\right), 4.63-4.27$ (m, $3 \mathrm{H}), 3.41-3.10(\mathrm{~m}, 3 \mathrm{H}), 2.39$ (s, $3 \mathrm{H}), 1.97-1.53$ (m, $4 \mathrm{H}), 1.45$ (s, $9 \mathrm{H})$.

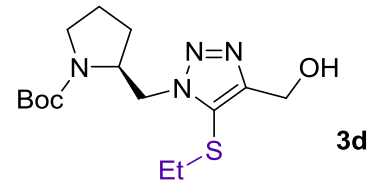


tert-Butyl (L)-2-((5-(ethylthio)-4-(hydroxymethyl)-1H-1,2,3-triazol-1-yl)methyl)pyrrolidine-1carboxylate (3d)

$\mathrm{Rf}=0.5(\mathrm{DCM} / \mathrm{MeOH}=20: 1)$.

${ }^{1} \mathbf{H}$ NMR $\left(400 \mathrm{MHz}, \mathrm{CDCl}_{3}\right) \delta 4.77(\mathrm{~d}, J=4.0 \mathrm{~Hz}, 2 \mathrm{H}), 4.68-4.31(\mathrm{~m}, 2 \mathrm{H}), 4.30-4.21(\mathrm{~m}, 1 \mathrm{H})$, 3.45-3.17 (m, $3 \mathrm{H}), 2.79$ (q, $J=8.0 \mathrm{~Hz}, 2 \mathrm{H}), 1.95-1.51$ (m, $4 \mathrm{H}), 1.45$ (s, $9 \mathrm{H}), 1.18$ (t, $J=8.0 \mathrm{~Hz}, 3$ $\mathrm{H})$.

ESI-MS: $m / z$ calcd. for $\mathrm{C}_{15} \mathrm{H}_{27} \mathrm{~N}_{4} \mathrm{O}_{3} \mathrm{~S}(\mathrm{M}+\mathrm{H})^{+} 343.1798$, found 343.1859 . 
III. Enantiomeric excess investigations on $N$-Boc- $(L)$-prolinol, 2 and triazole monomers
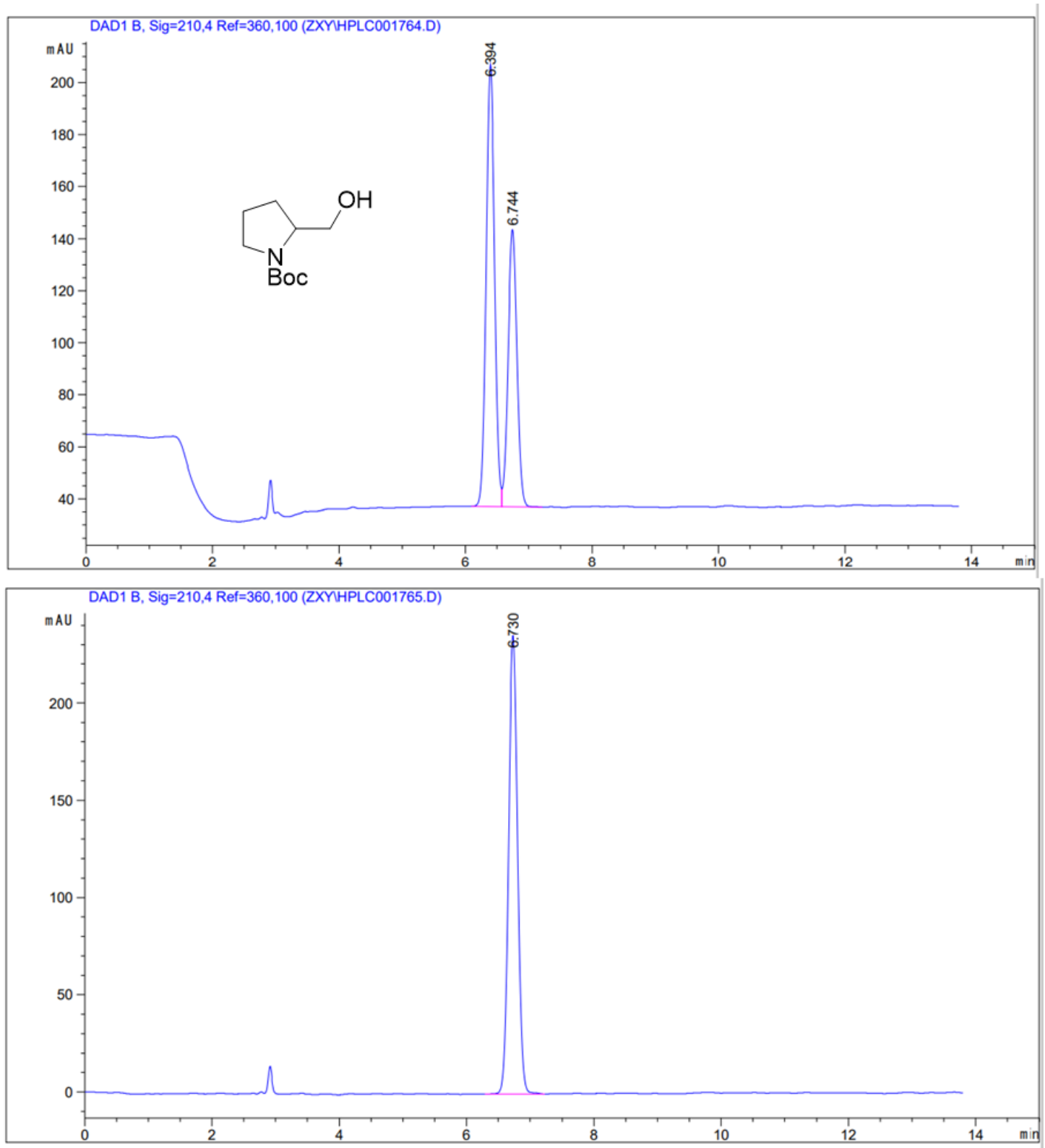

Figure S1. Chiral HPLC traces of racemic (up) and enantiomeric (down) $N$-Boc- $(L)$-prolinol in hexane/isopropanol $(\mathrm{v} / \mathrm{v}=95 / 5)$. 

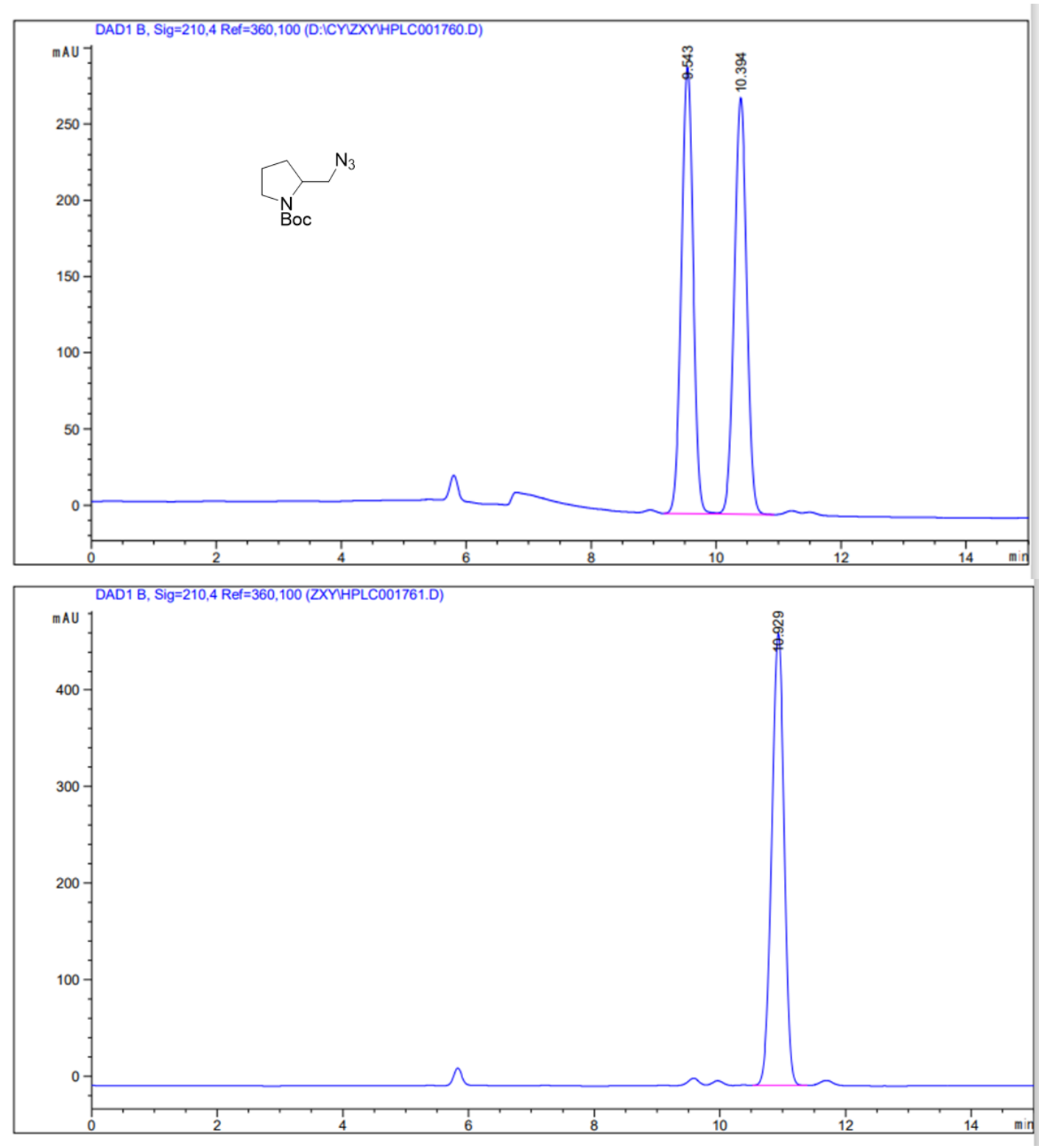

Figure S2. Chiral HPLC traces of racemic (up) and enantiomeric (down) 2 in hexane/isopropanol (v/v =99/1). 

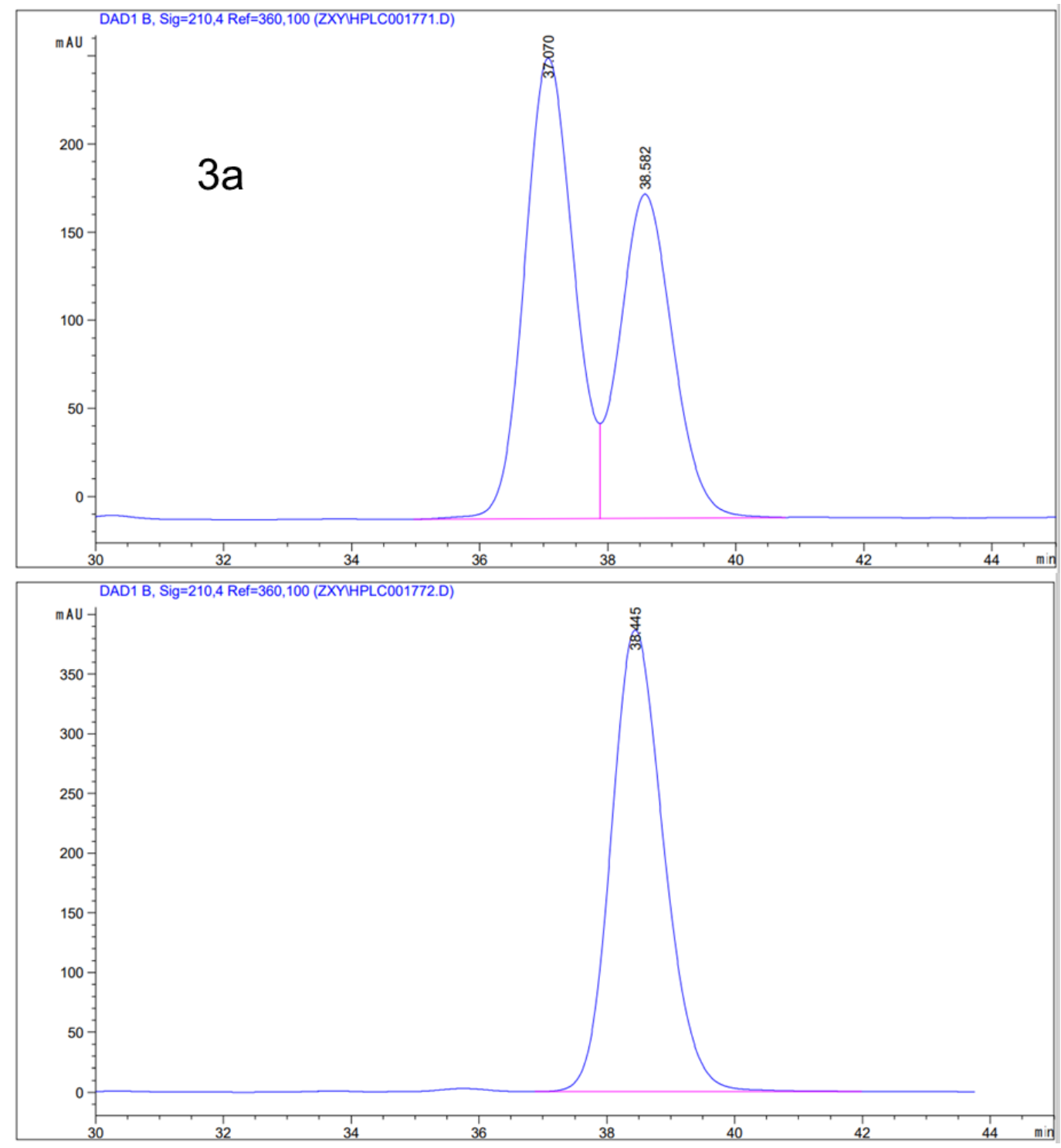

Figure S3. Chiral HPLC traces of racemic (up) and enantiomeric (down) 3a in hexane/isopropanol $(\mathrm{v} / \mathrm{v}=92 / 8)$. 


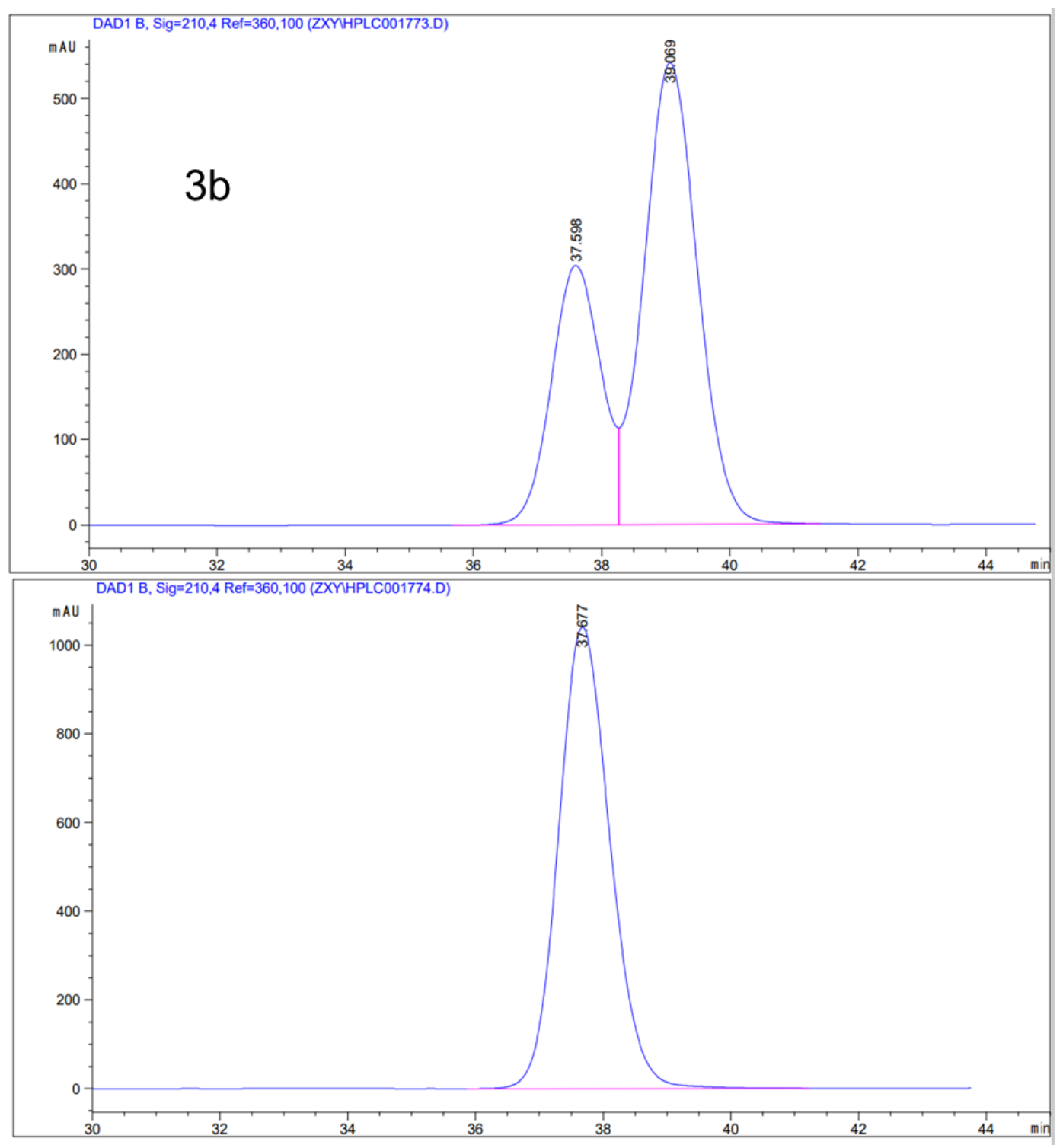

Figure S4. Chiral HPLC traces of racemic (up) and enantiomeric (down) $\mathbf{3 b}$ in hexane/isopropanol $(\mathrm{v} / \mathrm{v}=92 / 8)$. 

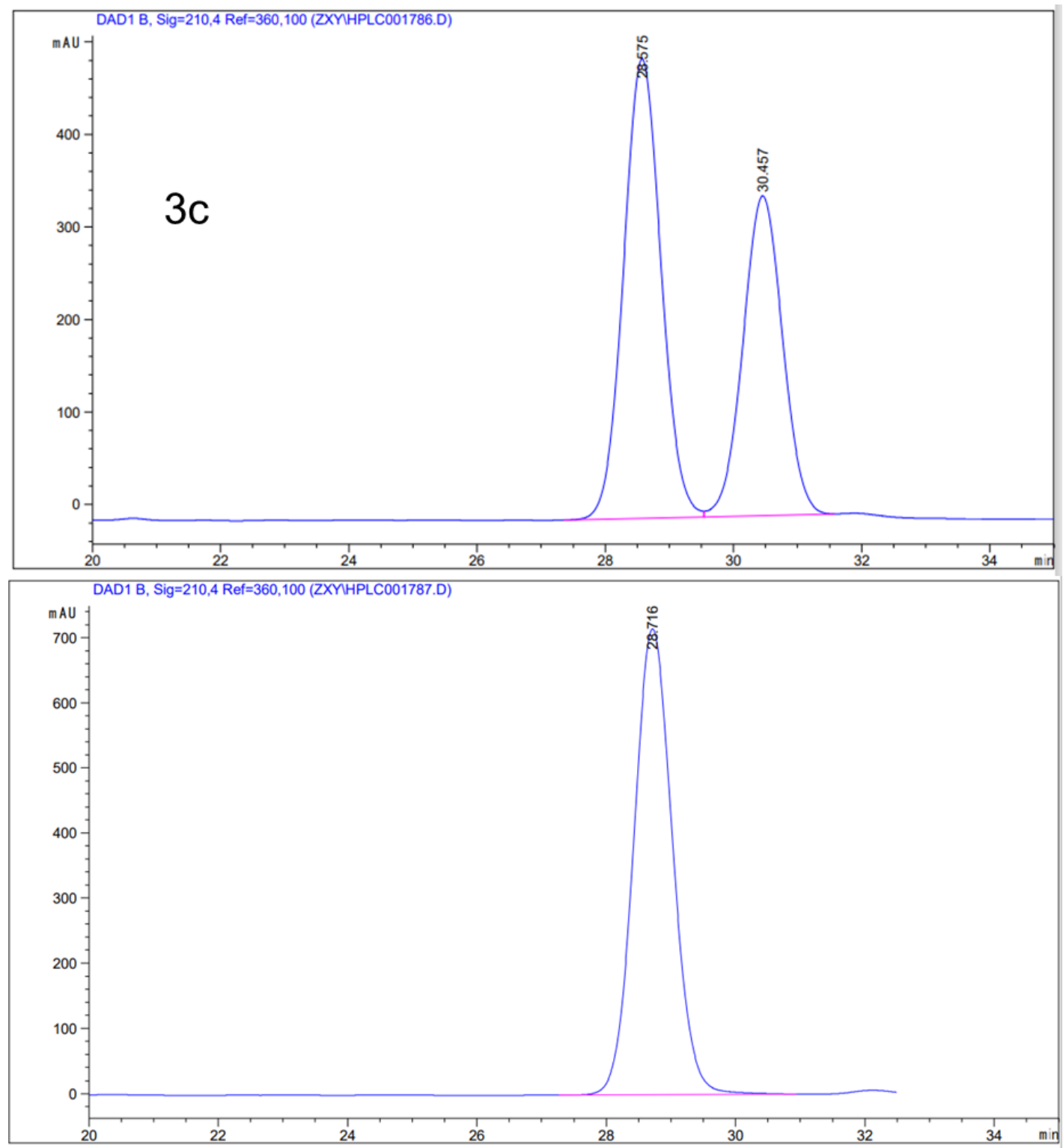

Figure S5. Chiral HPLC traces of racemic (up) and enantiomeric (down) 3c in hexane/isopropanol $(\mathrm{v} / \mathrm{v}=92 / 8)$. 
IV. UV/Vis spectra of $N$-Boc- $(L)$-prolinol, 2 and triazole monomers
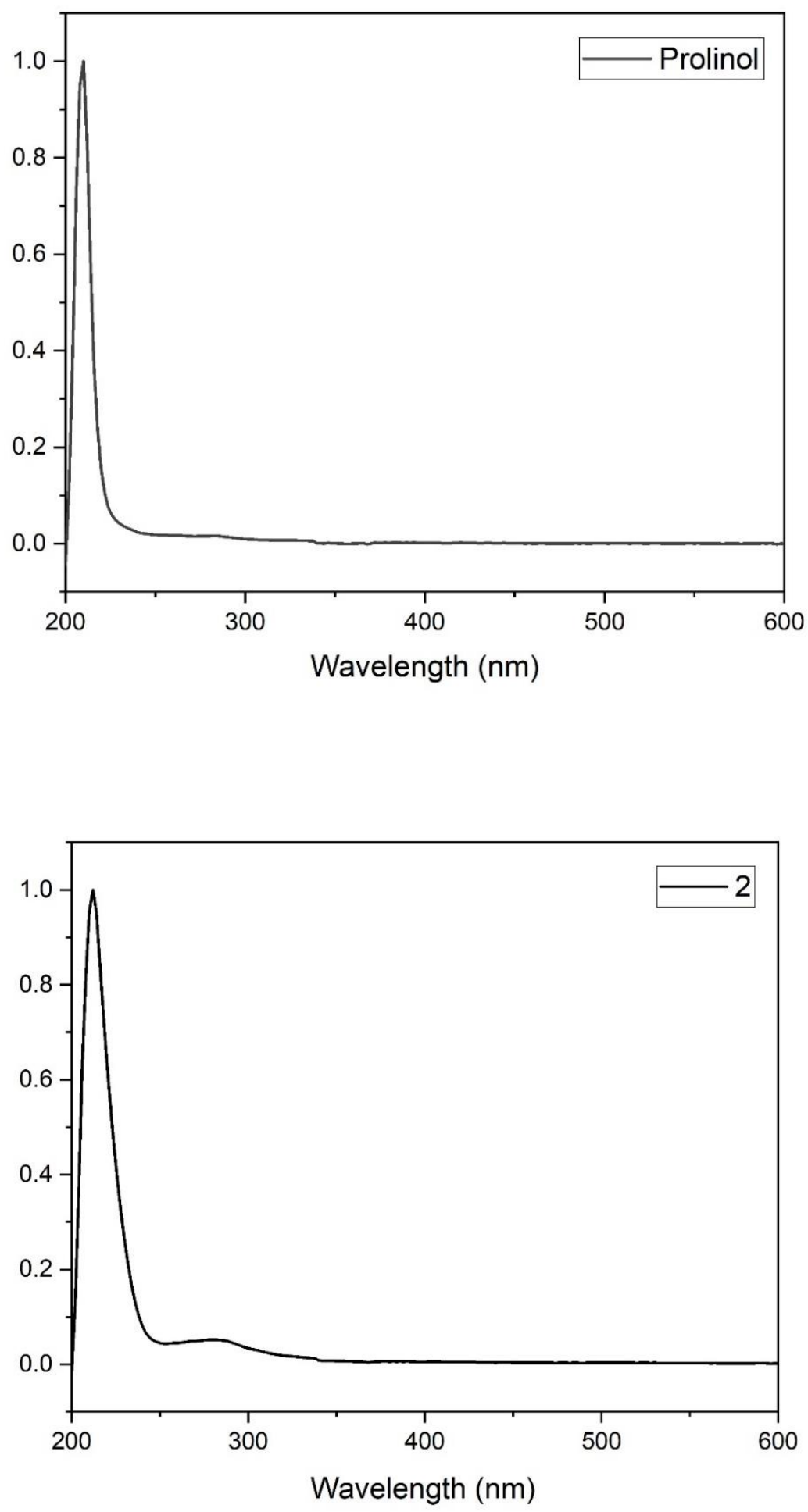

Figure S6. UV/Vis spectra of $N$-Boc-( $L$-prolinol (up) and 2 (down). 


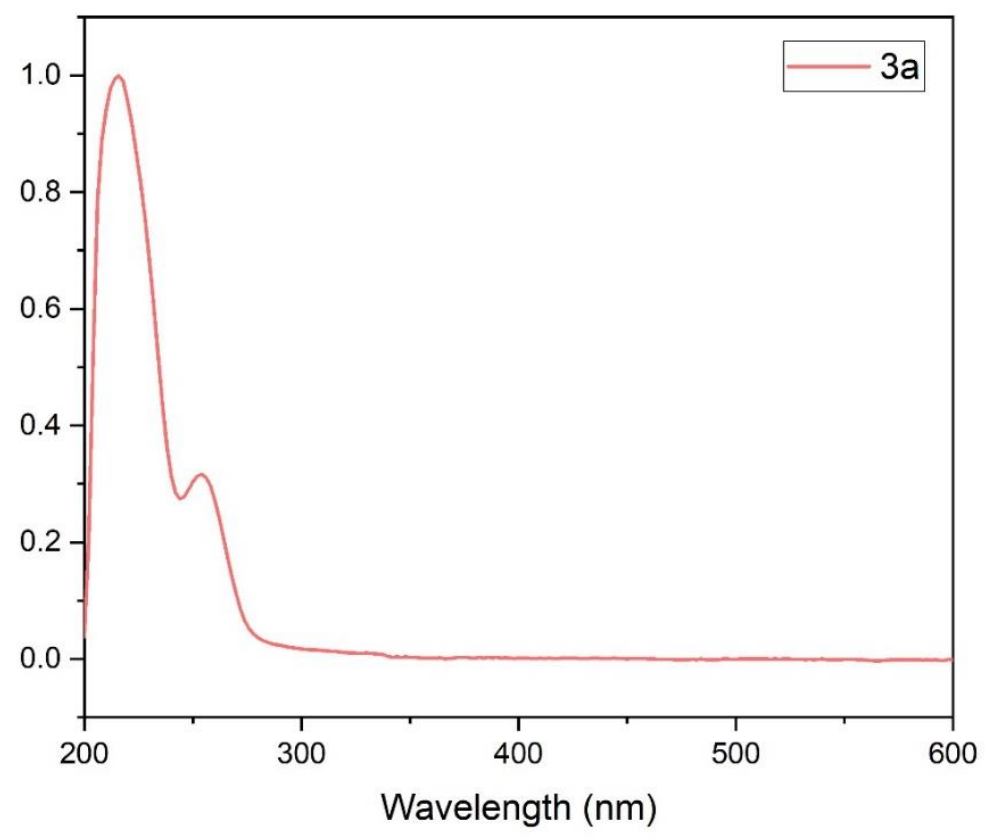

Figure S7. UV/Vis spectra of $\mathbf{3 a}$.

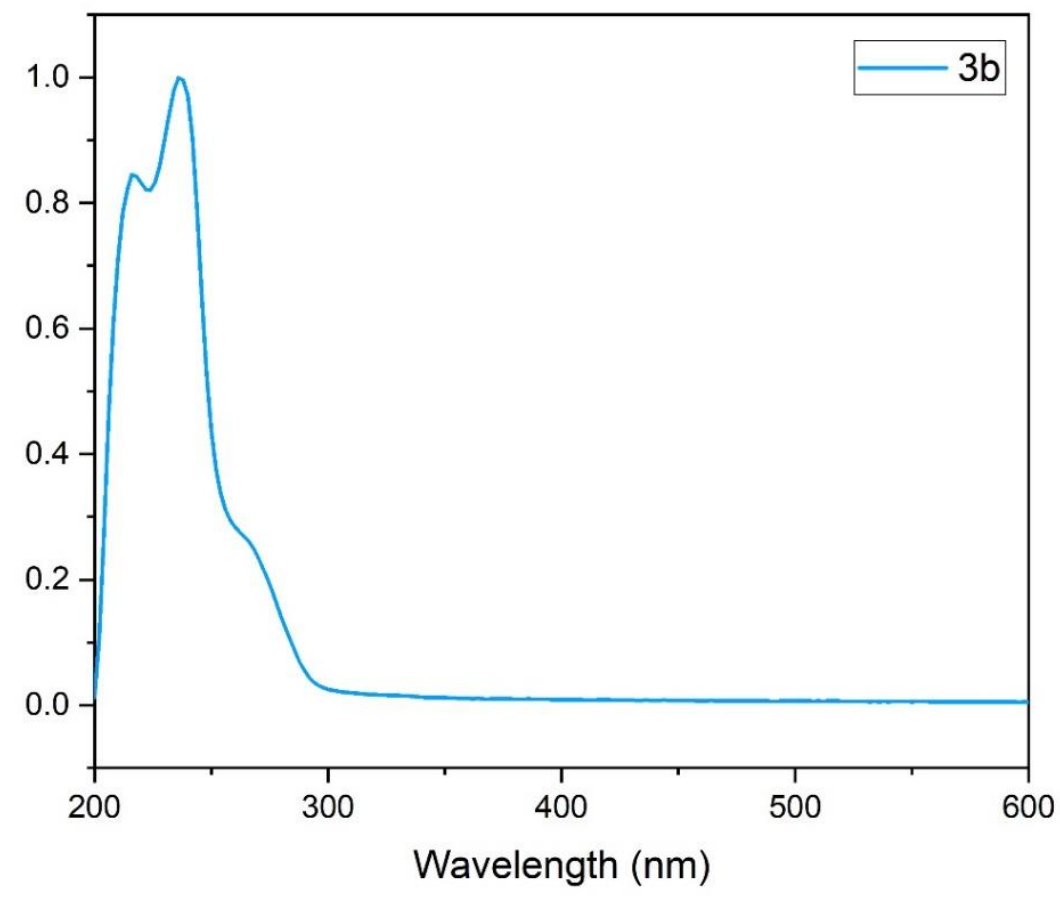

Figure S8. UV/Vis spectra of $\mathbf{3 b}$. 


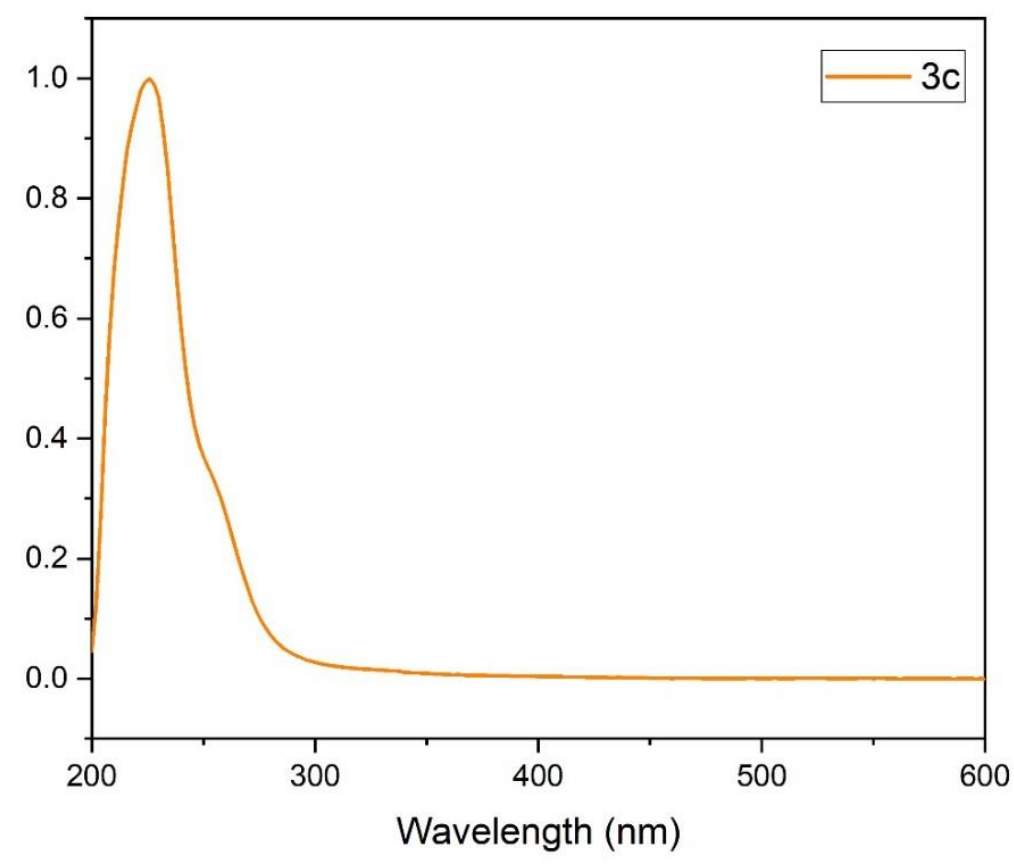

Figure S9. UV/Vis spectra of $\mathbf{3 c}$.

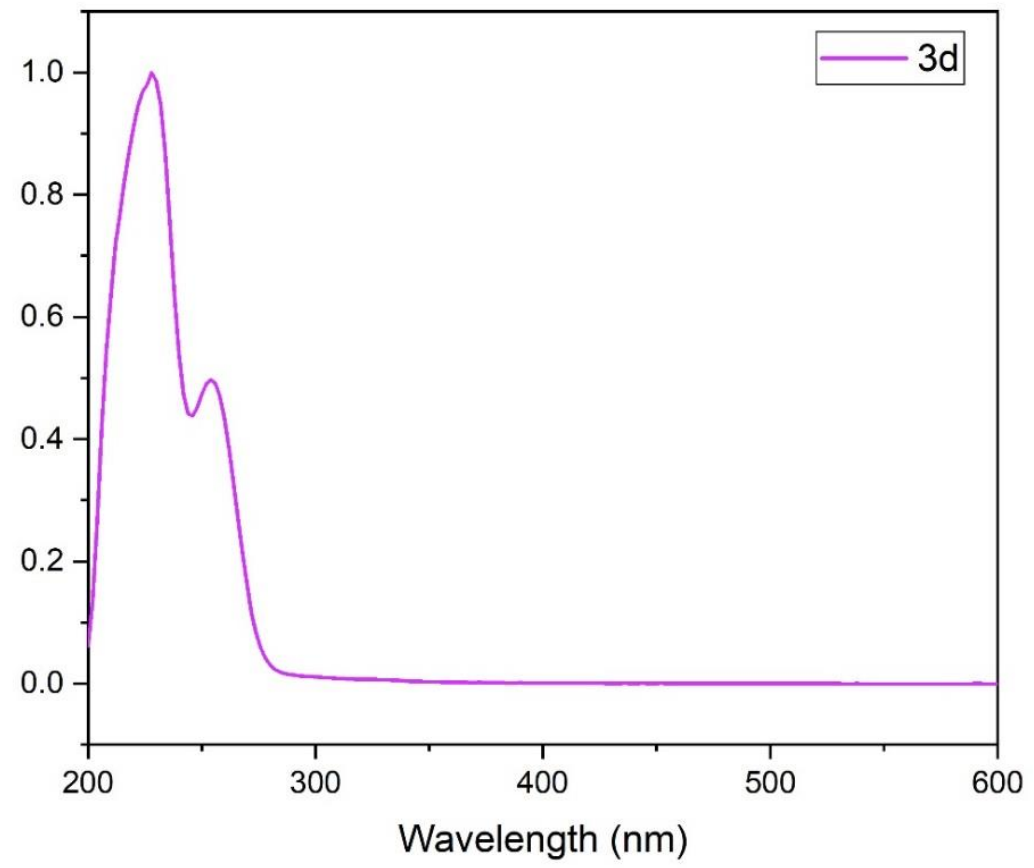

Figure S10. UV/Vis spectra of $\mathbf{3 d}$. 


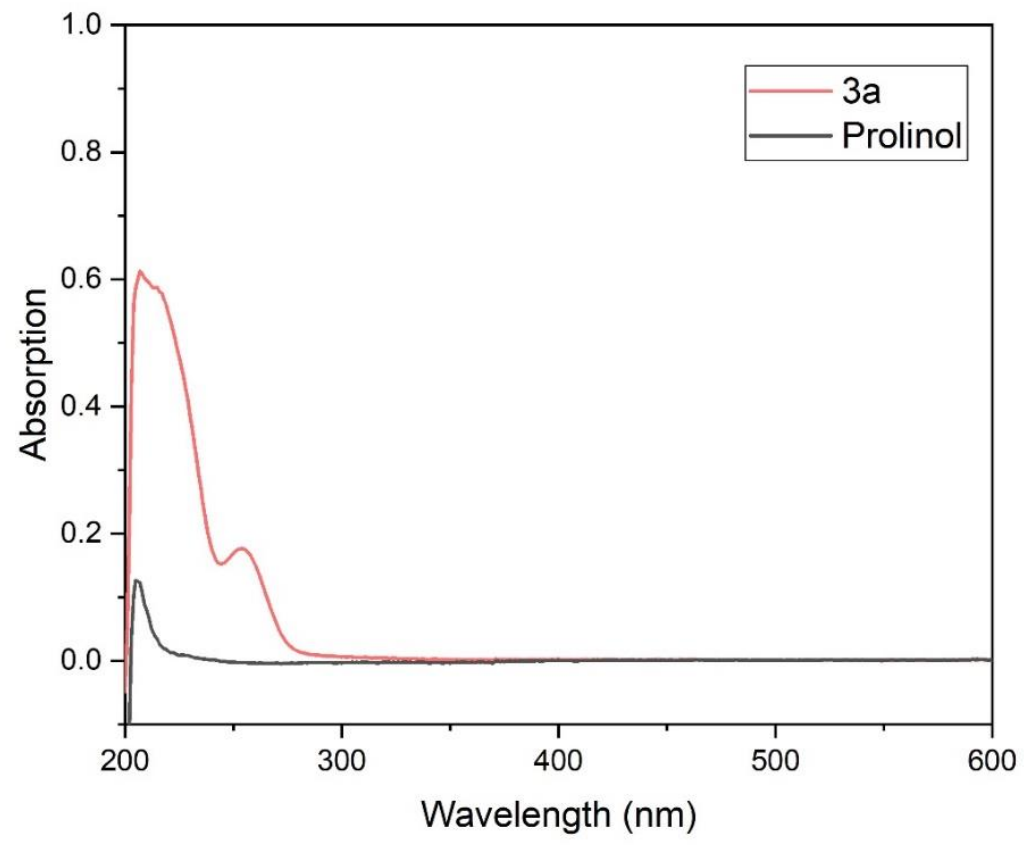

Figure S11. UV/Vis spectra of prolinol and 3a at the same concentration $\left(2.5 \times 10^{-4} \mathrm{M}\right.$ in methanol). 


\section{Synthesis and characterization of sequence-defined oligotriazoles}

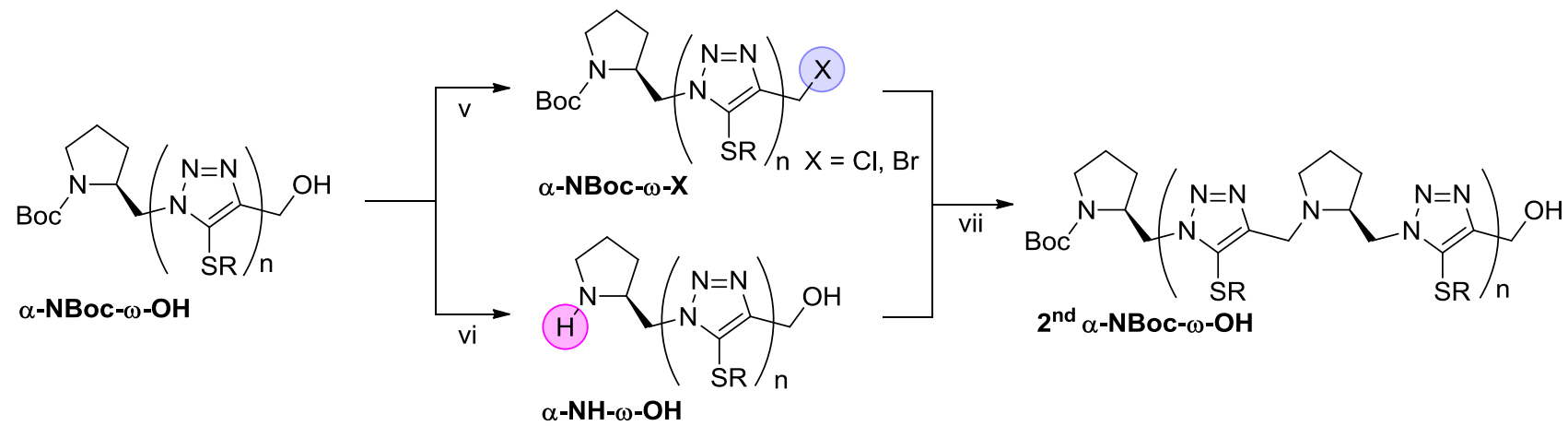

Scheme S2. Construction of chiral sequence-defined oligotriazoles

\section{General procedures}

v. Chlorination: at $0{ }^{\circ} \mathrm{C}$, compound $\boldsymbol{\alpha - N B o c}-\boldsymbol{\omega}-\mathbf{O H}$ and trimethylamine were dissolved in DCM $(0.5$ $\mathrm{M})$, then the solution of methanesulfonyl chloride ( $\mathrm{MsCl}, 2.0$ eq.) in DCM (1.5 M) was slowly added into the previous mixture. The reaction mixture was stirred at room temperature for $2-7 \mathrm{~h}$ until the reaction completed (confirmed by TLC), and then concentrated under reduced pressure. The residue was purified by silica gel flash column chromatography to give the desired product $\alpha$ NBoc- $\omega-\mathbf{C l}$.

Bromination: at $0{ }^{\circ} \mathrm{C}$, monomer 3 and triphenylphosphine $\left(\mathrm{PPh}_{3}, 2.0\right.$ eq.) were dissolved in $\mathrm{DCM}$ $(0.5 \mathrm{M})$, then the solution of $\mathrm{CBr}_{4}$ (2.0 eq.) in DCM (1.5 M) was slowly added into the previous mixture. The reaction mixture was stirred at room temperature for 10-30 min until the reaction completed (confirmed by TLC), and then concentrated under reduced pressure. The residue was purified by silica gel flash column chromatography to give the desired product $\boldsymbol{\alpha}-\mathbf{N B o c}-\boldsymbol{\omega}-\mathbf{B r}$.

vi. Deprotection of Boc group: at $0{ }^{\circ} \mathrm{C}$, compound $\alpha-\mathrm{NBoc}-\omega-\mathrm{OH}$ was dissolved in methanol $(0.5 \mathrm{M})$, then the solution of acetyl chloride ( $\mathrm{AcCl}, 3.0$ eq.) in $\mathrm{MeOH}(1.5 \mathrm{M})$ was slowly added into the previous mixture. The reaction mixture was stirred at room temperature until the reaction completed (confirmed by TLC), and then evaporated under vacuum. The residue was diluted with saturated $\mathrm{NaOH}$ solution, washed with $\mathrm{DCM}$ (three times), dried over $\mathrm{Na}_{2} \mathrm{SO}_{4}$, filtered, and evaporated under vacuum to give the crude product $\alpha-\mathbf{N H}-\omega-\mathbf{O H}$, which was then purified by silica gel column chromatography.

vii. Coupling: compound $\boldsymbol{\alpha}-\mathbf{N H}-\boldsymbol{\omega}-\mathbf{O H}$ was dissolved in acetonitrile $(0.5 \mathrm{M})$, then $\mathrm{K}_{2} \mathrm{CO}_{3}$ was added into the previous mixture. The reaction mixture was stirred at room temperature for 10 minutes. Then compound product $\boldsymbol{\alpha}$-NBoc- $\omega$-X and KI were added into the mixture. The reaction mixture was then heated to $60{ }^{\circ} \mathrm{C}$ for $0.5-4 \mathrm{~h}$ until the reaction completed, which was confirmed by TLC. 
The solution was then cooled to room temperature, then evaporated under vacuum. Then diluted with ethyl acetate, washed with brine (three times), dried over $\mathrm{Na}_{2} \mathrm{SO}_{4}$, filtered, and evaporated under vacuum. The crude product was purified by column chromatography on silica gel to afford the pure product $2^{\text {nd }} \boldsymbol{\alpha - N B o c - \omega - O H}$.

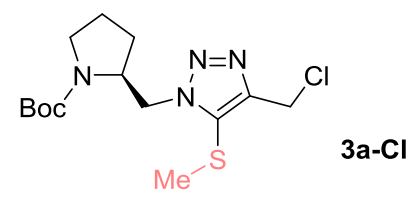

tert-Butyl (L)-2-((4-(chloromethyl)-5-(methylthio)-1H-1,2,3-triazol-1-yl)methyl)pyrrolidine-1carboxylate (3a-Cl)

$\mathrm{Rf}=0.5(\mathrm{PE} / \mathrm{EA}=1: 1)$.

${ }^{1} \mathbf{H}$ NMR $\left(400 \mathrm{MHz}, \mathrm{CDCl}_{3}\right) \delta 4.69(\mathrm{~s}, 2 \mathrm{H}), 4.62-4.23(\mathrm{~m}, 3 \mathrm{H}), 3.43-3.16(\mathrm{~m}, 2 \mathrm{H}), 2.34(\mathrm{~s}, 3 \mathrm{H})$, $1.92-1.54(\mathrm{~m}, 4 \mathrm{H}), 1.39$ (s, $9 \mathrm{H})$.

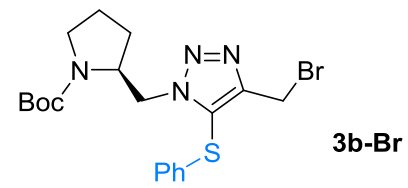

tert-Butyl (L)-2-((4-(bromomethyl)-5-(phenylthio)-1H-1,2,3-triazol-1-yl)methyl)pyrrolidine-1carboxylate $(3 \mathrm{~b}-\mathrm{Br})$

$\mathrm{Rf}=0.5(\mathrm{PE} / \mathrm{EA}=1: 1)$.

${ }^{1} \mathbf{H}$ NMR (400 MHz, $\left.\mathrm{CDCl}_{3}\right) \delta$ 7.36-7.13 (m, $\left.4 \mathrm{H}\right), 7.08-7.05(\mathrm{~m}, 1 \mathrm{H}), 4.51-4.26(\mathrm{~m}, 5 \mathrm{H}), 3.43-3.18$ (m, 2 H), 1.94-1.49 (m, 4 H), 1.47-1.40 (m, 9 H).

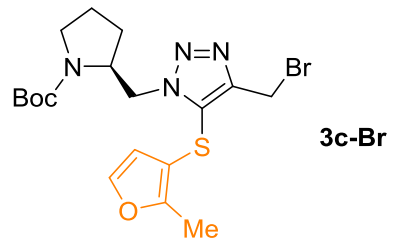

tert-Butyl

(L)-2-((4-(bromomethyl)-5-((2-methylfuran-3-yl)thio)-1H-1,2,3-triazol-1-

yl)methyl)pyrrolidine-1-carboxylate (3c-Br)

$\mathrm{Rf}=0.5(\mathrm{PE} / \mathrm{EA}=1: 1)$.

${ }^{1} \mathbf{H}$ NMR $\left(400 \mathrm{MHz}, \mathrm{CDCl}_{3}\right) \delta$ 7.25-7.23 (m, $\left.1 \mathrm{H}\right), 6.24-6.20(\mathrm{~m}, 1 \mathrm{H}), 4.62-4.49(\mathrm{~m}, 2 \mathrm{H}), 4.48-4.28$ (m, 3 H), 3.45-3.19 (m, 2 H), 2.42 (s, 3 H), 1.92-1.53 (m, 4 H), 1.45 (s, 9 H). 


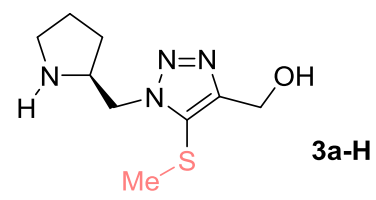

(L)-(5-(Methylthio)-1-(pyrrolidin-2-ylmethyl)-1H-1,2,3-triazol-4-yl)methanol (3a-H) $\mathrm{Rf}=0.2(\mathrm{DCM} / \mathrm{MeOH}=10: 1)$.

${ }^{1}$ H NMR $\left(400 \mathrm{MHz}, \mathrm{CDCl}_{3}\right) \delta 4.76(\mathrm{~s}, 2 \mathrm{H}), 4.38(\mathrm{~d}, J=8.0 \mathrm{~Hz}, 2 \mathrm{H}), 3.75-3.66(\mathrm{~m}, 1 \mathrm{H}), 3.05(\mathrm{~s}, 1$ H), 3.04-2.89 (m, 2 H), 2.36 (s, 3 H), 1.96-1.72 (m, 3 H), 1.59-1.48 (m, 1 H).

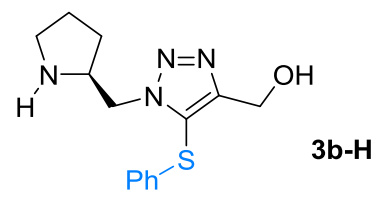

(L)-(5-(Phenylthio)-1-(pyrrolidin-2-ylmethyl)-1H-1,2,3-triazol-4-yl)methanol (3b-H)

$\mathrm{Rf}=0.2(\mathrm{DCM} / \mathrm{MeOH}=10: 1)$.

${ }^{1}$ H NMR (400 MHz, $\left.\mathrm{CDCl}_{3}\right) \delta$ 7.32-7.19 (m, $\left.3 \mathrm{H}\right), 7.11$ (d, J=4.0 Hz, $\left.2 \mathrm{H}\right), 4.75(\mathrm{~s}, 2 \mathrm{H}), 4.30-4.26$ (m, 2 H), 3.63-3.53 (m, $1 \mathrm{H}), 2.98-2.78$ (m, $3 \mathrm{H}), 1.86-1.63$ (m, $3 \mathrm{H}), 1.54-1.36$ (m, $1 \mathrm{H})$.

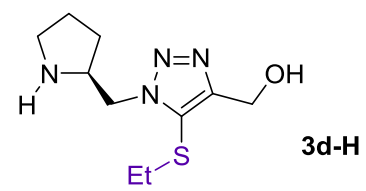

(L)-(5-(Ethylthio)-1-(pyrrolidin-2-ylmethyl)-1H-1,2,3-triazol-4-yl)methanol (3d-H) $\mathrm{Rf}=0.2(\mathrm{DCM} / \mathrm{MeOH}=10: 1)$.

${ }^{1}$ H NMR $\left(400 \mathrm{MHz} \mathrm{CDCl}_{3}\right) \delta 4.75(\mathrm{~s}, 2 \mathrm{H}), 4.41-4.28(\mathrm{~m}, 2 \mathrm{H}), 3.73-3.56(\mathrm{~m}, 1 \mathrm{H}), 3.00-2.85$ (m, 2 H), 2.82-2.72 (m, 2 H), 1.94-1.62 (m, $3 \mathrm{H}), 1.53-1.43(\mathrm{~m}, 1 \mathrm{H}), 1.18(\mathrm{t}, J=8.0 \mathrm{~Hz}, 3 \mathrm{H})$.

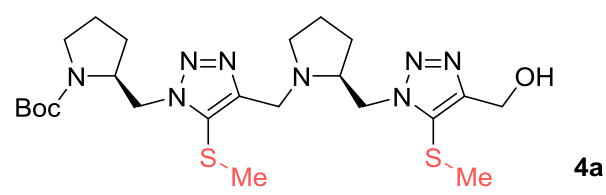

4a was prepared as white solid from 3a-Cl (5.5 mmol, $1.91 \mathrm{~g}, 1$ eq.) and 3a-H (6.6 mmol, $1.51 \mathrm{~g}, 1.1$ eq.) in $85 \%$ yield $(2.52 \mathrm{~g})$.

$\mathrm{Rf}=0.3(\mathrm{DCM} / \mathrm{MeOH}=20: 1)$.

${ }^{1} \mathbf{H}$ NMR $\left(400 \mathrm{MHz}, \mathrm{CDCl}_{3}\right) \delta 4.77$ (s, $\left.2 \mathrm{H}\right), 4.64-4.23(\mathrm{~m}, 5 \mathrm{H}), 3.96-3.90$ (m, $\left.1 \mathrm{H}\right), 3.73-3.71$ (m, 1 H), 3.62-3.19 (m, 4 H), 2.94 (s, 1 H), 2.52 (s, 1 H), 2.39 (s, 3 H), 2.35-2.30 (m, 3 H), 1.99-1.55 (m, 8 $\mathrm{H}), 1.43$ (s, $9 \mathrm{H})$.

ESI-MS $m / z$ calcd. for $\mathrm{C}_{23} \mathrm{H}_{39} \mathrm{~N}_{8} \mathrm{O}_{3} \mathrm{~S}_{2}(\mathrm{M}+\mathrm{H})^{+}$539.2581, found 539.2593. 


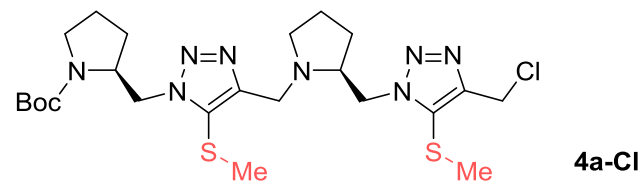

4a-Cl was prepared as white solid from $4 \mathbf{a}(2.5 \mathrm{mmol}, 2.05 \mathrm{~g}, 1.0$ eq.) in $65 \%$ yield $(1.01 \mathrm{~g})$.

$\mathrm{Rf}=0.6(\mathrm{DCM} / \mathrm{MeOH}=20: 1)$.

${ }^{1}$ H NMR (400 MHz, $\left.\mathrm{CDCl}_{3}\right) \delta 4.72(\mathrm{~s}, 2 \mathrm{H}), 4.63-4.22(\mathrm{~m}, 5 \mathrm{H}), 4.02-3.96$ (m, $\left.1 \mathrm{H}\right), 3.76-3.71$ (m, 1 H), 3.44-3.16 (m, 3 H), 3.00-2.92 (m, $1 \mathrm{H}), 2.56-2.48(\mathrm{~m}, 1 \mathrm{H}), 2.37$ (s, 3H), 2.32-2.26 (m, $3 \mathrm{H})$, $1.98-1.53(\mathrm{~m}, 8 \mathrm{H}), 1.43(\mathrm{~s}, 9 \mathrm{H})$.

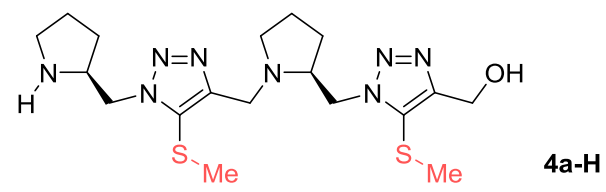

4a-H was prepared as white solid from $4 \mathbf{a}$ (1.8 mmol, $0.97 \mathrm{~g}, 1.0$ eq.) in $95 \%$ yield ( $0.75 \mathrm{~g})$. $\mathrm{Rf}=0.2(\mathrm{DCM} / \mathrm{MeOH}=10: 1)$.

${ }^{1} \mathbf{H}$ NMR $\left(400 \mathrm{MHz}, \mathrm{CDCl}_{3}\right) \delta 4.72$ (s, $\left.2 \mathrm{H}\right), 4.45-4.38$ (m, $\left.1 \mathrm{H}\right), 4.32-4.24$ (m, 3 H), 3.89-3.73 (m, 1 H), 3.69-3.63 (m, 2 H), 3.43-3.31 (m, 1 H), 3.07-2.84 (m, 4 H), 2.58-2.50 (m, $1 \mathrm{H}), 2.34$ (s, $3 \mathrm{H})$, 2.27 (s, $3 \mathrm{H}), 1.92-1.64(\mathrm{~m}, 7 \mathrm{H}), 1.52-1.44(\mathrm{~m}, 1 \mathrm{H})$.

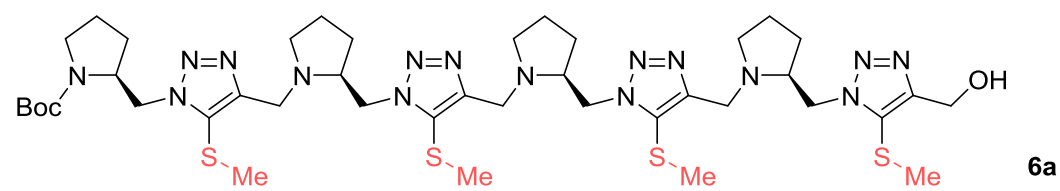

6a was prepared as white solid from 4a-Cl (1.5 mmol, $0.84 \mathrm{~g}, 1$ eq.) and 4a-H (1.8 mmol, $0.79 \mathrm{~g}, 1.2$ eq.) in $64 \%$ yield $(0.92 \mathrm{~g})$.

$\mathrm{Rf}=0.2(\mathrm{DCM} / \mathrm{MeOH}=15: 1)$.

${ }^{1} \mathbf{H}$ NMR $\left(400 \mathrm{MHz}, \mathrm{CDCl}_{3}\right) \delta 4.80(\mathrm{~s}, 2 \mathrm{H}), 4.69-4.48(\mathrm{~m}, 4 \mathrm{H}), 4.40-4.28(\mathrm{~m}, 4 \mathrm{H}), 4.17-4.04$ (m, 2 H), 3.94-3.90 (m, $1 \mathrm{H}), 3.83-3.69$ (m, $3 \mathrm{H}), 3.46-3.24$ (m, $5 \mathrm{H}), 3.06-2.78$ (m, $4 \mathrm{H}), 2.60-2.52$ (m, 3 H), 2.40 (s, $3 \mathrm{H}), 2.37$ (s, $3 \mathrm{H}), 2.35$ (s, $6 \mathrm{H}), 1.90-1.64$ (m, $16 \mathrm{H}), 1.46$ (s, $9 \mathrm{H})$.

ESI-MS $m / z$ calcd. for $\mathrm{C}_{41} \mathrm{H}_{67} \mathrm{~N}_{16} \mathrm{O}_{3} \mathrm{~S}_{4}(\mathrm{M}+\mathrm{H})^{+}$959.4459, found 959.4482.

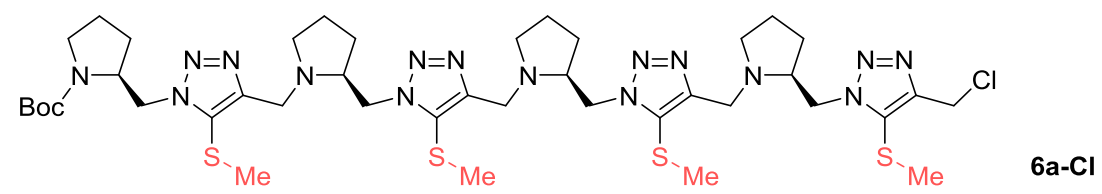

6a-Cl was prepared as white solid from $6 \mathbf{a}(0.6 \mathrm{mmol}, 0.58 \mathrm{~g}, 1.0 \mathrm{eq}$.$) in 43 \%$ yield $(0.25 \mathrm{~g})$. 
$\mathrm{Rf}=0.4(\mathrm{DCM} / \mathrm{MeOH}=20: 1)$.

${ }^{1}$ H NMR (400 MHz, CDCl $) \delta 4.76(\mathrm{~s}, 2 \mathrm{H}), 4.67-4.25(\mathrm{~m}, 9 \mathrm{H}), 4.09-4.02(\mathrm{~m}, 3 \mathrm{H}), 3.82-3.74(\mathrm{~m}, 3$ H), 3.45-3.23 (m, 5 H), 3.04-2.96 (m, 3 H), 2.60-2.50 (m, 3 H), 2.40 (s, 3 H), 2.36 (s, 3 H), 2.34 (s, 6 H), 2.07-1.66 (m, $16 \mathrm{H}), 1.47$ (s, $9 \mathrm{H})$.

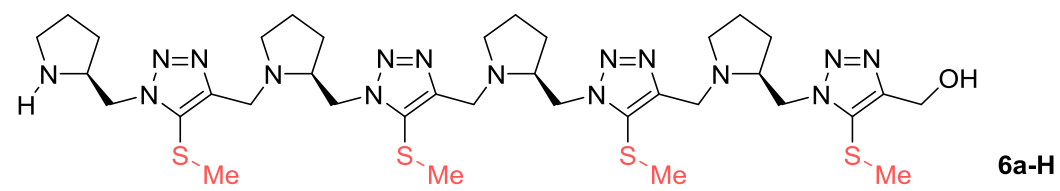

6a-H was prepared as white solid from $6 \mathbf{a}(0.35 \mathrm{mmol}, 0.29 \mathrm{~g}, 1.0 \mathrm{eq}$ ) in $83 \%$ yield $(0.25 \mathrm{~g})$. $\mathrm{Rf}=0.3(\mathrm{DCM} / \mathrm{MeOH}=5: 1)$.

${ }^{1} \mathbf{H}$ NMR $\left(400 \mathrm{MHz}, \mathrm{CDCl}_{3}\right) \delta 4.78(\mathrm{~s}, 2 \mathrm{H}), 4.56-4.43(\mathrm{~m}, 3 \mathrm{H}), 4.36-4.24$ (m, $\left.5 \mathrm{H}\right), 4.05-3.99$ (m, 2 H), 3.92-3.88 (m, $1 \mathrm{H}), 3.81-3.63(\mathrm{~m}, 4 \mathrm{H}), 3.38-3.25(\mathrm{~m}, 3 \mathrm{H}), 3.02-2.87(\mathrm{~m}, 5 \mathrm{H}), 2.65(\mathrm{~s}, 1 \mathrm{H})$, 2.55-2.50 (m, $3 \mathrm{H}), 2.36(\mathrm{~s}, 3 \mathrm{H}), 2.33$ (s, $3 \mathrm{H}), 2.31$ (s, $6 \mathrm{H}), 1.93-1.85$ (m, $1 \mathrm{H}), 1.83-1.64$ (m, 14 $\mathrm{H}), 1.57-1.46(\mathrm{~m}, 1 \mathrm{H})$.

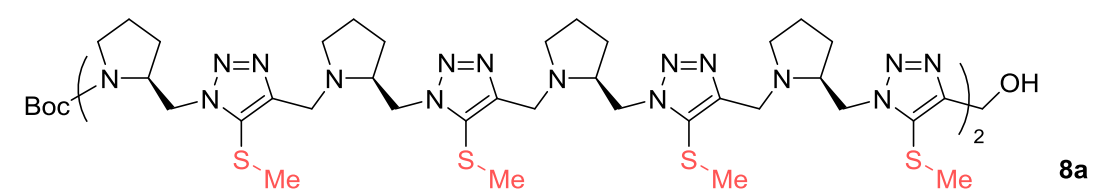

8a was prepared as white solid from 6a-Cl (0.2 mmol, 0.2 g, 1 eq. $)$ and $\mathbf{6 a - H}(0.21 \mathrm{mmol}, 0.18 \mathrm{~g}, 1.05$ eq.) in $87 \%$ yield $(0.31 \mathrm{~g})$.

$\mathrm{Rf}=0.2(\mathrm{DCM} / \mathrm{MeOH}=10: 1)$.

${ }^{1} \mathbf{H}$ NMR $\left(400 \mathrm{MHz}, \mathrm{CDCl}_{3}\right) \delta 4.74(\mathrm{~s}, 2 \mathrm{H}), 4.59-4.41(\mathrm{~m}, 8 \mathrm{H}), 4.32-4.20$ (m, $\left.8 \mathrm{H}\right), 4.05-3.86$ (m, 7 H), 3.77-3.65 (m, $7 \mathrm{H}), 3.30-3.20(\mathrm{~m}, 9 \mathrm{H}), 3.00-2.82(\mathrm{~m}, 7 \mathrm{H}), 2.50-2.43(\mathrm{~m}, 7 \mathrm{H}), 2.33(\mathrm{~s}, 3 \mathrm{H})$, 2.30 (s, $18 \mathrm{H}), 2,28$ (s, $3 \mathrm{H}), 1.93-1.49$ (m, $32 \mathrm{H}), 1.39$ (s, $9 \mathrm{H})$.

MALDI-TOF-MS $m / z$ calcd. for $\mathrm{C}_{77} \mathrm{H}_{122} \mathrm{~N}_{32} \mathrm{O}_{3} \mathrm{~S}_{8}(\mathrm{M})^{+} 1799.8$, found 1799.9.

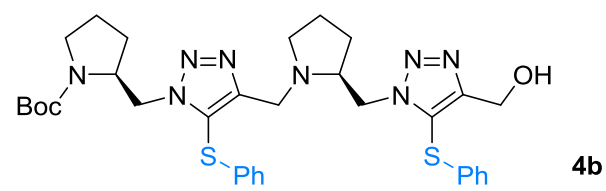

4b was prepared as white solid from $\mathbf{3 b}-\mathbf{B r}$ (3.47 mmol, $1.57 \mathrm{~g}, 1$ eq.) and $\mathbf{3 b}-\mathbf{H}$ (3.82 mmol, $1.11 \mathrm{~g}$, 1.1 eq.) in $93 \%$ yield $(2.15 \mathrm{~g})$.

$\mathrm{Rf}=0.3(\mathrm{DCM} / \mathrm{MeOH}=15: 1)$.

${ }^{1} \mathbf{H}$ NMR $\left(400 \mathrm{MHz}, \mathrm{CDCl}_{3}\right) \delta$ 7.25-7.13 (m, $\left.6 \mathrm{H}\right), 7.06-7.00(\mathrm{~m}, 3 \mathrm{H}), 6.91-6.87(\mathrm{~m}, 1 \mathrm{H}), 4.73(\mathrm{~d}$, $J=8.0 \mathrm{~Hz}, 2 \mathrm{H}), 4.54-4.26$ (m, $4 \mathrm{H}), 4.23-4.07$ (m, 1 H), 3.82-3.63 (m, $2 \mathrm{H}), 3.39-3.09$ (m, $3 \mathrm{H})$, 3.07-2.82 (m, 2 H), 2.43-2.35 (m, 1 H), 1.90-1.52 (m, 8 H), 1.46-1.40 (m, 9 H). 
ESI-MS $m / z$ calcd. for $\mathrm{C}_{33} \mathrm{H}_{43} \mathrm{~N}_{8} \mathrm{O}_{3} \mathrm{~S}_{2}(\mathrm{M}+\mathrm{H})^{+}$663.2894, found 663.2971.

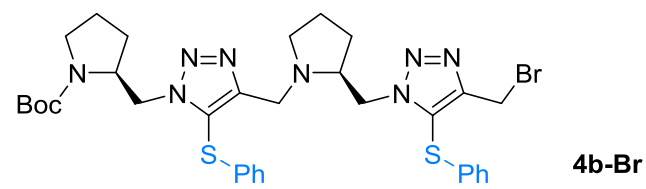

4b-Br was prepared as white solid from $4 \mathbf{b}$ (1.5 mmol, $0.99 \mathrm{~g}, 1.0$ eq.) in 56\% yield ( $0.60 \mathrm{~g})$. $\mathrm{Rf}=0.2(\mathrm{PE} / \mathrm{EA}=2: 1)$.

${ }^{1} \mathbf{H}$ NMR $\left(400 \mathrm{MHz}, \mathrm{CDCl}_{3}\right) \delta$ 7.23-6.84 (m, $\left.10 \mathrm{H}\right), 4.46(\mathrm{~s}, 2 \mathrm{H}), 4.40-4.16(\mathrm{~m}, 3 \mathrm{H}), 3.86-3.80$ (m, $1 \mathrm{H}), 3.61-3.51(\mathrm{~m}, 1 \mathrm{H}), 3.43-3.06(\mathrm{~m}, 3 \mathrm{H}), 2.90-2.83(\mathrm{~m}, 1 \mathrm{H}), 2.42-2.32(\mathrm{~m}, 1 \mathrm{H}), 1.86-1.45$ (m, $8 \mathrm{H}), 1.41-1.38(\mathrm{~m}, 9 \mathrm{H})$.

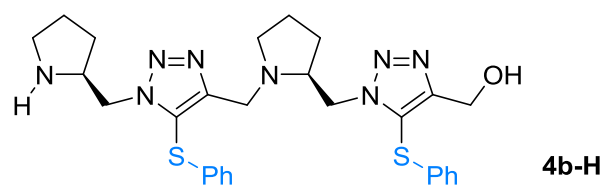

4b-H was prepared as white solid from $4 \mathbf{b}$ (1.5 mmol, $0.99 \mathrm{~g}, 1.0$ eq.) in 99\% yield (0.75 g). $\mathrm{Rf}=0.3(\mathrm{DCM} / \mathrm{MeOH}=10: 1)$.

${ }^{1} \mathbf{H}$ NMR $\left(400 \mathrm{MHz}, \mathrm{CDCl}_{3}\right) \delta 7.22-7.08(\mathrm{~m}, 6 \mathrm{H}), 6.99(\mathrm{~d}, J=8.0 \mathrm{~Hz}, 2 \mathrm{H}), 6.93(\mathrm{~d}, J=8.0 \mathrm{~Hz}, 2 \mathrm{H})$, 4.66 (s, 2 H), 4.35-4.15 (m, 3 H), 4.11-4.05 (m, 1 H), 3.81-3.77 (m, 1 H), 3.64-3.51 (m, 2 H), 3.18$3.09(\mathrm{~m}, 1 \mathrm{H}), 3.03-2.73(\mathrm{~m}, 4 \mathrm{H}), 2.46-2.36$ (m, $1 \mathrm{H}), 1.83-1.51(\mathrm{~m}, 7 \mathrm{H}), 1.46-1.30$ (m, $1 \mathrm{H})$.

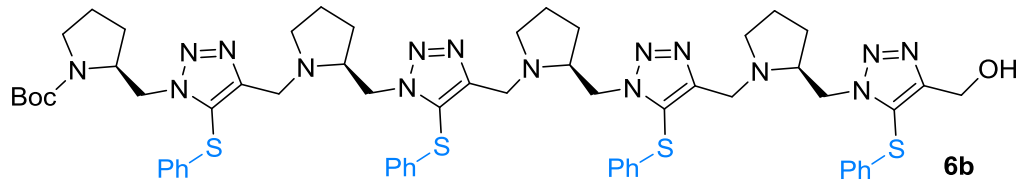

6b was prepared as white solid from $\mathbf{4 b - B r}(0.41 \mathrm{mmol}, 0.30 \mathrm{~g}, 1$ eq. $)$ and $\mathbf{4 b}-\mathbf{H}(0.45 \mathrm{mmol}, 0.25 \mathrm{~g}$, 1.1 eq.) in $69 \%$ yield $(0.32 \mathrm{~g})$.

$\mathrm{Rf}=0.2(\mathrm{DCM} / \mathrm{MeOH}=15: 1)$.

${ }^{1} \mathbf{H}$ NMR $\left(400 \mathrm{MHz}, \mathrm{CDCl}_{3}\right) \delta$ 7.22-7.10 (m, $\left.12 \mathrm{H}\right), 7.03-7.00(\mathrm{~m}, 3 \mathrm{H}), 6.92-6.88(\mathrm{~m}, 5 \mathrm{H}), 4.72(\mathrm{~s}$, $2 \mathrm{H}), 4.55-4.45(\mathrm{~m}, 1 \mathrm{H}), 4.39-4.22(\mathrm{~m}, 5 \mathrm{H}), 4.18-4.05$ (m, $3 \mathrm{H}), 3.90-3.74$ (m, $3 \mathrm{H}), 3.68-3.52$ (m, $3 \mathrm{H}), 3.34-3.12$ (m, $5 \mathrm{H}), 2.88-2.74(\mathrm{~m}, 4 \mathrm{H}), 2.49-2.28$ (m, $3 \mathrm{H}), 1.91-1.47$ (m, $16 \mathrm{H}), 1.44-1.42$ (m, $9 \mathrm{H})$.

ESI-MS $m / z$ calcd. for $\mathrm{C}_{61} \mathrm{H}_{75} \mathrm{~N}_{16} \mathrm{O}_{3} \mathrm{~S}_{4}(\mathrm{M}+\mathrm{H})^{+}$1207.5085, found 1207.5107.

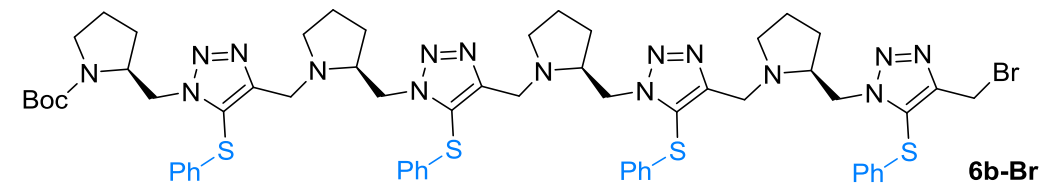


6b-Br was prepared as white solid from $6 \mathbf{b}(0.26 \mathrm{mmol}, 0.31 \mathrm{~g}, 1.0$ eq.) in $52 \%$ yield $(0.17 \mathrm{~g})$. $\mathrm{Rf}=0.2(\mathrm{PE} / \mathrm{EA}=1: 2)$.

${ }^{1} \mathbf{H}$ NMR $\left(400 \mathrm{MHz}, \mathrm{CDCl}_{3}\right) \delta$ 7.21-7.00 (m, $\left.15 \mathrm{H}\right), 6.91-6.88(\mathrm{~m}, 5 \mathrm{H}), 4.50-4.21$ (m, $\left.7 \mathrm{H}\right), 4.12-$ 4.08 (m, 3 H), 3.90-3.82 (m, 3 H), 3.63-3.56 (m, 3 H), 3.36-3.06 (m, 5 H), 2.89-2.80 (m, 3 H), 2.402.35 (m, $3 \mathrm{H}), 1.83-1.45$ (m, $16 \mathrm{H}), 1.43-1.39$ (m, $9 \mathrm{H})$.

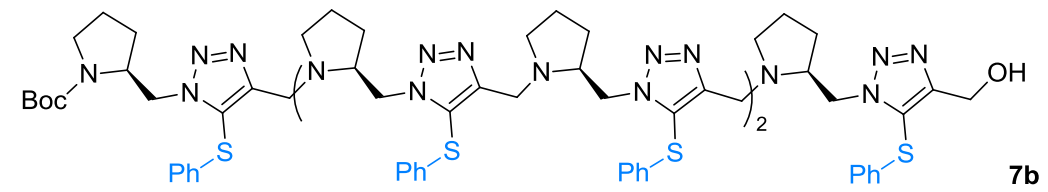

7b was prepared as white solid from $\mathbf{6 b}-\mathbf{B r}(0.11 \mathrm{mmol}, 0.14 \mathrm{~g}, 1$ eq. $)$ and $\mathbf{4 b}-\mathbf{H}(0.12 \mathrm{mmol}, 0.07 \mathrm{~g}$, 1.1 eq.) in $73 \%$ yield $(0.14 \mathrm{~g})$.

$\mathrm{Rf}=0.2(\mathrm{DCM} / \mathrm{MeOH}=10: 1)$.

${ }^{1}$ H NMR (400 MHz, $\left.\mathrm{CDCl}_{3}\right) \delta$ 7.25-7.12 (m, $\left.18 \mathrm{H}\right), 7.03-7.00(\mathrm{~m}, 3 \mathrm{H}), 6.92-6.88(\mathrm{~m}, 9 \mathrm{H}), 4.72(\mathrm{~s}$, $2 \mathrm{H}), 4.52-4.31$ (m, 8 H), 4.10-4.02 (m, 5 H), 3.90-3.75 (m, 5 H), 3.64-3.55 (m, 5 H), 3.40-3.10 (m, $6 \mathrm{H}), 2.90-2.84$ (m, $6 \mathrm{H}), 2.46-2.29$ (m, $5 \mathrm{H}), 1.85-1.52$ (m, $24 \mathrm{H}), 1.43-1.41$ (m, $9 \mathrm{H})$.

MALDI-TOF-MS $m / z$ calcd. for $\mathrm{C}_{89} \mathrm{H}_{106} \mathrm{~N}_{24} \mathrm{O}_{3} \mathrm{~S}_{6}(\mathrm{M})^{+} 1751.7$, found 1751.6.

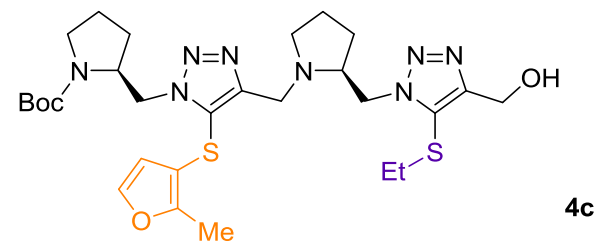

4c was prepared as white solid from 3c-Br (2.7 mmol, $1.23 \mathrm{~g}, 1$ eq.) and 3d-H (3.2 mmol, $0.78 \mathrm{~g}, 1.2$ eq.) in $82 \%$ yield $(1.36 \mathrm{~g})$.

$\mathrm{Rf}=0.3(\mathrm{DCM} / \mathrm{MeOH}=20: 1)$.

${ }^{1} \mathbf{H}$ NMR $\left(400 \mathrm{MHz}, \mathrm{CDCl}_{3}\right) \delta 7.19(\mathrm{~s}, 1 \mathrm{H}), 6.25-6.11(\mathrm{~m}, 1 \mathrm{H}), 4.79-4.77$ (m, $\left.2 \mathrm{H}\right), 4.60-4.25$ (m, 5 H), 4.00-3.86 (m, 1 H), 3.82-3.65 (m, $1 \mathrm{H}), 3.41-3.20$ (m, $3 \mathrm{H}), 3.07-2.89(\mathrm{~m}, 2 \mathrm{H}), 2.78$ (q, $J=8.0$ Hz, $2 \mathrm{H}), 2.48-2.44(\mathrm{~m}, 1 \mathrm{H}), 2.38$ (s, $3 \mathrm{H}), 2.05-1.64(\mathrm{~m}, 8 \mathrm{H}), 1.46$ (s, $9 \mathrm{H}), 1.18$ (t, J = 8.0 Hz, 3 $\mathrm{H})$.

ESI-MS $m / z$ calcd. for $\mathrm{C}_{28} \mathrm{H}_{43} \mathrm{~N}_{8} \mathrm{O}_{4} \mathrm{~S}_{2}(\mathrm{M}+\mathrm{H})^{+} 619.2841$, found 619.2871.

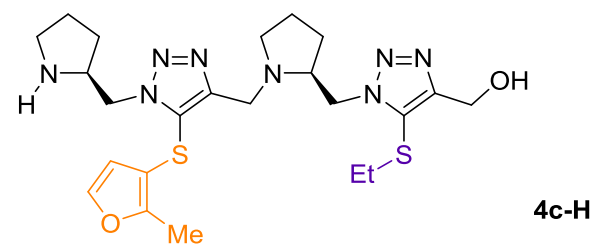

4c-H was prepared as white solid from $4 \mathbf{c}(2.1 \mathrm{mmol}, 1.30 \mathrm{~g}, 1.0 \mathrm{eq}$.$) in 98 \%$ yield $(1.07 \mathrm{~g})$. 
$\mathrm{Rf}=0.2(\mathrm{DCM} / \mathrm{MeOH}=10: 1)$.

${ }^{1}$ H NMR (400 MHz, CDCl 3$) \delta 7.19$ (s, $\left.1 \mathrm{H}\right), 6.14$ (s, $\left.1 \mathrm{H}\right), 4.74$ (s, $\left.2 \mathrm{H}\right), 4.47-4.42$ (m, $\left.1 \mathrm{H}\right), 4.32-$ 4.20 (m, 3 H), 3.94-3.90 (m, $1 \mathrm{H}), 3.71-3.59$ (m, 2 H), 3.38-3.28 (m, $1 \mathrm{H}), 3.13-2.82$ (m, $4 \mathrm{H}), 2.76$ $(\mathrm{q}, J=8.0 \mathrm{~Hz}, 2 \mathrm{H}), 2.54-2.52(\mathrm{~m}, 1 \mathrm{H}), 2.35(\mathrm{~s}, 3 \mathrm{H}), 1.89-1.67(\mathrm{~m}, 7 \mathrm{H}), 1.51-1.38(\mathrm{~m}, 1 \mathrm{H}), 1.17$ (t, $J=8.0 \mathrm{~Hz}, 3 \mathrm{H})$.

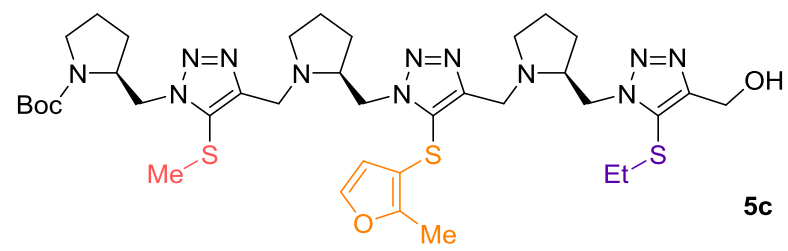

5c was prepared as white solid from 4c-H (2.0 mmol, $1.04 \mathrm{~g}, 1$ eq. $)$ and 3a-Cl (2.0 mmol, $0.69 \mathrm{~g}, 1.0$ eq.) in $84 \%$ yield $(1.39 \mathrm{~g})$.

$\mathrm{Rf}=0.2(\mathrm{DCM} / \mathrm{MeOH}=20: 1)$.

${ }^{1}$ H NMR (400 MHz, $\left.\mathrm{CDCl}_{3}\right) \delta 7.21$ (s, $\left.1 \mathrm{H}\right), 6.14$ (s, $\left.1 \mathrm{H}\right), 4.77$ (s, $\left.2 \mathrm{H}\right), 4.61-4.45$ (m, $\left.4 \mathrm{H}\right), 4.32-$ 4.27 (m, 3 H), 4.03-3.91 (m, 2 H), 3.76-3.70 (m, 2 H), 3.43-3.18 (m, 4 H), 3.07-2.87 (m, 3 H), 2.77 (q, $J=8.0 \mathrm{~Hz}, 2 \mathrm{H}), 2.53-2.43(\mathrm{~m}, 2 \mathrm{H}), 2.36(\mathrm{~s}, 3 \mathrm{H}), 2.33-2.31(\mathrm{~m}, 3 \mathrm{H}), 1.96-1.55(\mathrm{~m}, 12 \mathrm{H}), 1.45$ $(\mathrm{s}, 9 \mathrm{H}), 1.17(\mathrm{t}, J=8.0 \mathrm{~Hz}, 3 \mathrm{H})$.

ESI-MS $m / z$ calcd. for $\mathrm{C}_{37} \mathrm{H}_{57} \mathrm{~N}_{12} \mathrm{O}_{4} \mathrm{~S}_{3}(\mathrm{M}+\mathrm{H})^{+} 829.3782$, found 829.3800 .

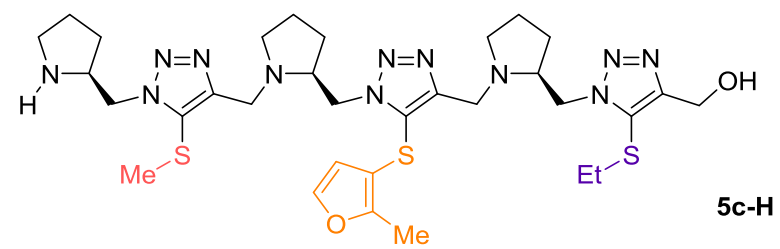

5c-H was prepared as white solid from $5 \mathbf{c}(1.6 \mathrm{mmol}, 1.30 \mathrm{~g}, 1.0$ eq. $)$ in $99 \%$ yield $(1.15 \mathrm{~g})$.

$\mathrm{Rf}=0.2(\mathrm{DCM} / \mathrm{MeOH}=10: 1)$.

${ }^{1} \mathbf{H}$ NMR $\left(400 \mathrm{MHz}, \mathrm{CDCl}_{3}\right) \delta 7.21$ (s, $\left.1 \mathrm{H}\right), 6.15$ (s, $\left.1 \mathrm{H}\right), 4.77$ (s, $\left.2 \mathrm{H}\right), 4.54-4.43$ (m, $\left.2 \mathrm{H}\right), 4.34-$ 4.24 (m, 4 H), 4.04-3.99 (m, 1 H), 3.94-3.91 (m, 1 H), 3.76-3.64 (m, 3 H), 3.38-3.26 (m, 2 H), 3.002.88 (m, 4 H), 2.77 (q, $J=8.0$ Hz, 2 H), 2.53-2.48 (m, 2 H), 2.37 (s, 3 H), 2.31 (s, 3 H), 1.90-1.62 (m, $11 \mathrm{H}), 1.55-1.47(\mathrm{~m}, 1 \mathrm{H}), 1.17$ (t, $J=8.0 \mathrm{~Hz}, 3 \mathrm{H})$.

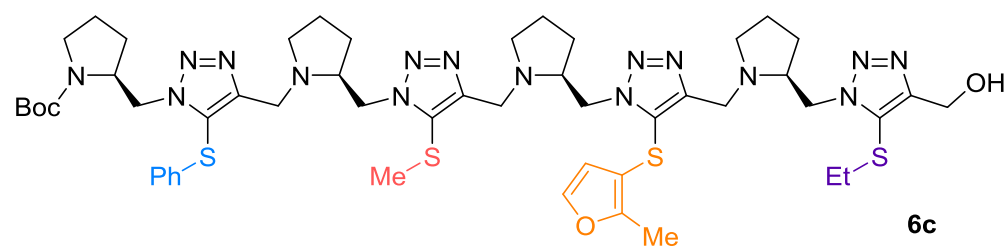


6c was prepared as white solid from $\mathbf{5 c - H}(1.6 \mathrm{mmol}, 1.15 \mathrm{~g}, 1$ eq. $)$ and $3 \mathrm{~b}-\mathbf{B r}(1.7 \mathrm{mmol}, 0.77 \mathrm{~g}, 1.05$ eq.) in $82 \%$ yield $(1.44 \mathrm{~g})$.

$\mathrm{Rf}=0.3(\mathrm{DCM} / \mathrm{MeOH}=15: 1)$.

${ }^{1} \mathbf{H}$ NMR $\left(400 \mathrm{MHz}, \mathrm{CDCl}_{3}\right) \delta 7.24-7.16(\mathrm{~m}, 4 \mathrm{H}), 7.08(\mathrm{~d}, J=8.0 \mathrm{~Hz}, 1 \mathrm{H}), 6.97(\mathrm{~d}, J=8.0 \mathrm{~Hz}, 1 \mathrm{H})$, 6.15 (s, $1 \mathrm{H}), 4.80-4.78$ (m, $2 \mathrm{H}), 4.56-4.27$ (m, 9 H), 4.10-3.98 (m, 2 H), 3.93-3.91 (m, $1 \mathrm{H}), 3.82-$ $3.68(\mathrm{~m}, 3 \mathrm{H}), 3.38-3.19(\mathrm{~m}, 5 \mathrm{H}), 3.03-2.87$ (m, $3 \mathrm{H}), 2.78$ (q, $J=8.0 \mathrm{~Hz}, 2 \mathrm{H}), 2.56-2.41$ (m, $4 \mathrm{H})$, $2.37(\mathrm{~s}, 3 \mathrm{H}), 2.28(\mathrm{~s}, 3 \mathrm{H}), 1.83-1.61(\mathrm{~m}, 16 \mathrm{H}), 1.46-1.44(\mathrm{~m}, 9 \mathrm{H}), 1.19(\mathrm{t}, J=8.0 \mathrm{~Hz}, 3 \mathrm{H})$.

ESI-MS $m / z$ calcd. for $\mathrm{C}_{51} \mathrm{H}_{73} \mathrm{~N}_{16} \mathrm{O}_{4} \mathrm{~S}_{4}(\mathrm{M}+\mathrm{H})^{+} 1101.4878$, found 1101.4890 .

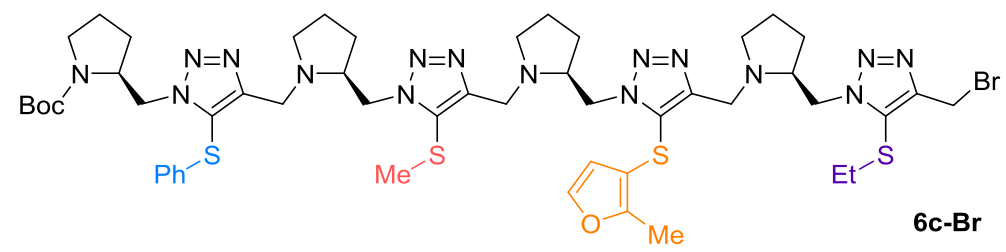

6c-Br was prepared as white solid from $6 \mathbf{c}(0.7 \mathrm{mmol}, 0.77 \mathrm{~g}, 1.0$ eq.) in $70 \%$ yield $(0.57 \mathrm{~g})$. $\mathrm{Rf}=0.2(\mathrm{DCM} / \mathrm{MeOH}=20: 1)$.

${ }^{1} \mathbf{H}$ NMR (400 MHz, $\left.\mathrm{CDCl}_{3}\right) \delta$ 7.25-7.17 (m, $\left.4 \mathrm{H}\right), 7.10-6.80(\mathrm{~m}, 2 \mathrm{H}), 6.13(\mathrm{~s}, 1 \mathrm{H}), 4.55(\mathrm{~s}, 2 \mathrm{H})$, 4.51-4.10 (m, 8 H), 4.09-3.60 (m, 6 H), 3.29-3.23 (m, 6 H), 3.02-2.89 (m, $2 \mathrm{H}), 2.84$ (q, J=8.0 Hz, 2H), 2.62-2.39 (m, 4 H), $2.34(\mathrm{~s}, 3 \mathrm{H}), 2.25(\mathrm{~s}, 3 \mathrm{H}), 1.87-1.49(\mathrm{~m}, 14 \mathrm{H}), 1.40$ (s, $9 \mathrm{H}), 1.30-1.24(\mathrm{~m}$, $2 \mathrm{H}), 1.19(\mathrm{t}, J=8.0 \mathrm{~Hz}, 3 \mathrm{H})$.

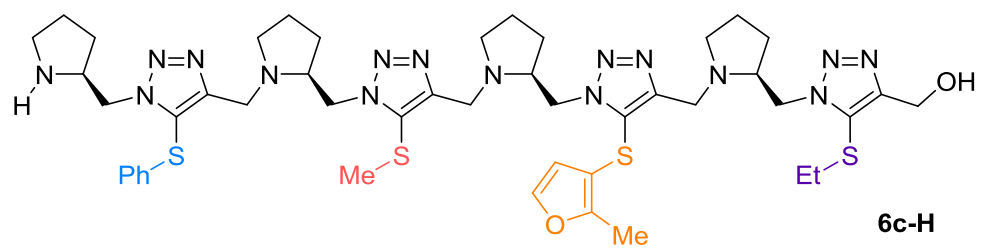

6c-H was prepared as white solid from $6 \mathbf{c}(0.6 \mathrm{mmol}, 0.66 \mathrm{~g}, 1.0$ eq.) in 77\% yield (0.46 g).

$\mathrm{Rf}=0.2(\mathrm{DCM} / \mathrm{MeOH}=10: 1)$.

${ }^{1}$ H NMR (400 MHz, $\left.\mathrm{CDCl}_{3}\right) \delta$ 7.26-7.14 (m, $\left.4 \mathrm{H}\right), 7.03-7.01$ (m, $\left.2 \mathrm{H}\right), 6.14(\mathrm{~s}, 1 \mathrm{H}), 4.76(\mathrm{~s}, 2 \mathrm{H})$, 4.53-4.39 (m, 3 H), 4.36-4.25 (m, 4 H), 4.17-4.10 (m, 1 H), 4.07-3.88 (m, 3 H), 3.77-3.66 (m, 3 H), 3.62-2.60 (m, $1 \mathrm{H}), 3.34-3.22(\mathrm{~m}, 3 \mathrm{H}), 3.01-2.88(\mathrm{~m}, 6 \mathrm{H}), 2.76$ (q, $J=8.0 \mathrm{~Hz}, 2 \mathrm{H}), 2.50-2.46(\mathrm{~m}$, $3 \mathrm{H}), 2.35(\mathrm{~s}, 3 \mathrm{H}), 2.27(\mathrm{~s}, 3 \mathrm{H}), 1.86-1.58(\mathrm{~m}, 15 \mathrm{H}), 1.51-1.42(\mathrm{~m}, 1 \mathrm{H}), 1.16(\mathrm{t}, J=8.0 \mathrm{~Hz}, 3 \mathrm{H})$.

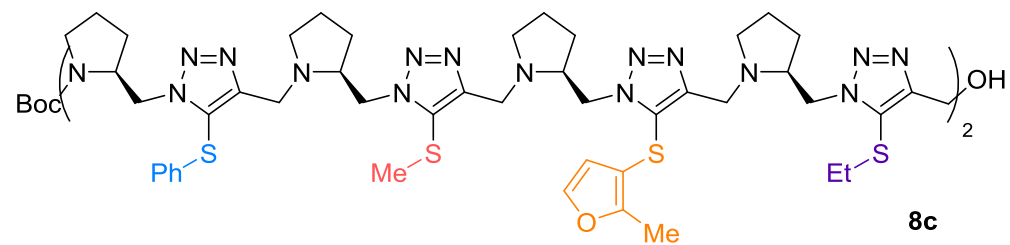


8c was prepared as white solid from $\mathbf{6 c - B r}(0.2 \mathrm{mmol}, 0.21 \mathrm{~g}, 1$ eq. $)$ and $\mathbf{6 c - H}(0.2 \mathrm{mmol}, 0.2 \mathrm{~g}, 1.0$ eq.) in $75 \%$ yield $(0.41 \mathrm{~g})$.

$\mathrm{Rf}=0.2(\mathrm{DCM} / \mathrm{MeOH}=10: 1)$.

${ }^{1} \mathbf{H}$ NMR $\left(400 \mathrm{MHz}, \mathrm{CDCl}_{3}\right) \delta$ 7.23-7.13 (m, $\left.8 \mathrm{H}\right), 7.03-7.00(\mathrm{~m}, 3 \mathrm{H}), 6.94-6.92(\mathrm{~m}, 1 \mathrm{H}), 6.15$ (s, 1 $\mathrm{H}), 6.13(\mathrm{~s}, 1 \mathrm{H}), 4.76(\mathrm{~s}, 2 \mathrm{H}), 4.51-4.40(\mathrm{~m}, 8 \mathrm{H}), 4.32-4.23(\mathrm{~m}, 6 \mathrm{H}), 4.20-4.16(\mathrm{~m}, 2 \mathrm{H}), 4.04-3.92$ (m, $6 \mathrm{H}), 3.78-3.62(\mathrm{~m}, 7 \mathrm{H}), 3.38-3.11(\mathrm{~m}, 10 \mathrm{H}), 3.00-2.84(\mathrm{~m}, 7 \mathrm{H}), 2.78-2.64(\mathrm{~m}, 4 \mathrm{H}), 2.48-2.40$ (m, $6 \mathrm{H}), 2.34(\mathrm{~s}, 6 \mathrm{H}), 2.25(\mathrm{~s}, 6 \mathrm{H}), 1.76-1.50(\mathrm{~m}, 32 \mathrm{H}), 1.42-1.40(\mathrm{~m}, 9 \mathrm{H}), 1.15$ (t, $J=8.0 \mathrm{~Hz}, 3$ $\mathrm{H}), 1.08(\mathrm{t}, J=8.0 \mathrm{~Hz}, 3 \mathrm{H})$.

MALDI-TOF-MS $m / z$ calcd. for $\mathrm{C}_{97} \mathrm{H}_{134} \mathrm{~N}_{32} \mathrm{O}_{5} \mathrm{~S}_{8}(\mathrm{M})^{+}$2083.9, found 2084.0. 


\section{Possible mechanism for MS/MS fragmentation}

(A) inductive cleavage of $\mathrm{C}-\mathrm{N}$ bond

i)

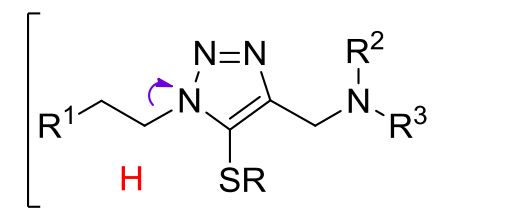

$[\mathrm{M}+\mathrm{H}]^{+}$

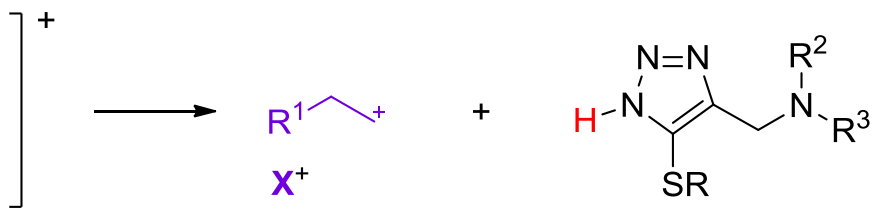

ii)

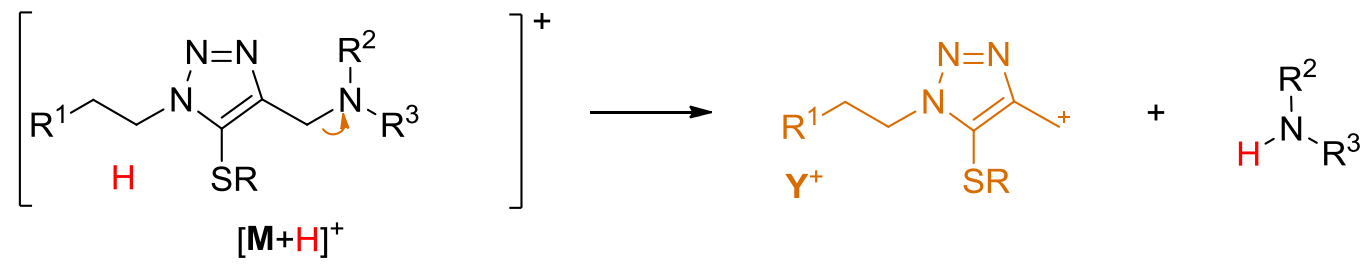

(B) rearrangement of $\mathrm{Y}^{+}$
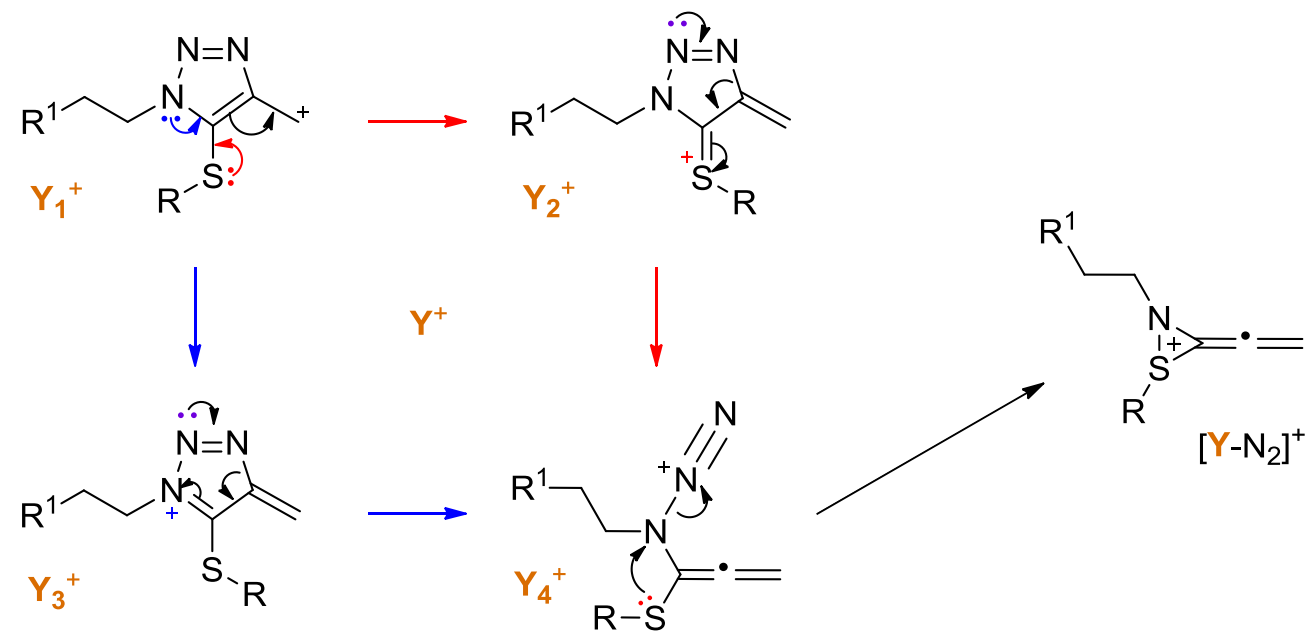

Scheme S3. Possible mechanism for MS/MS fragmentation. 


\section{NMR Spectra}
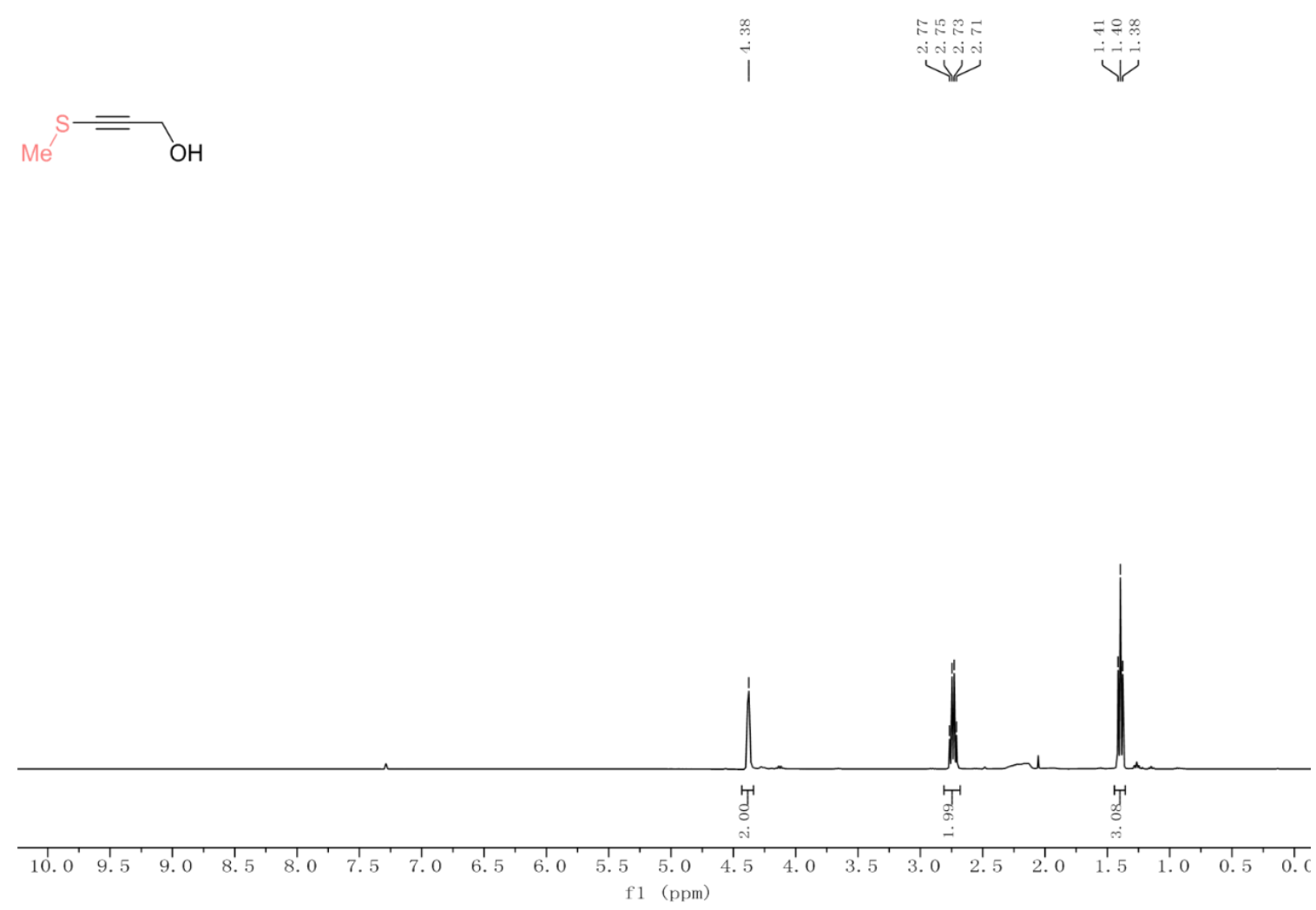

Figure S12. ${ }^{1} \mathrm{H}$ NMR spectra of $\mathbf{1 a}$.
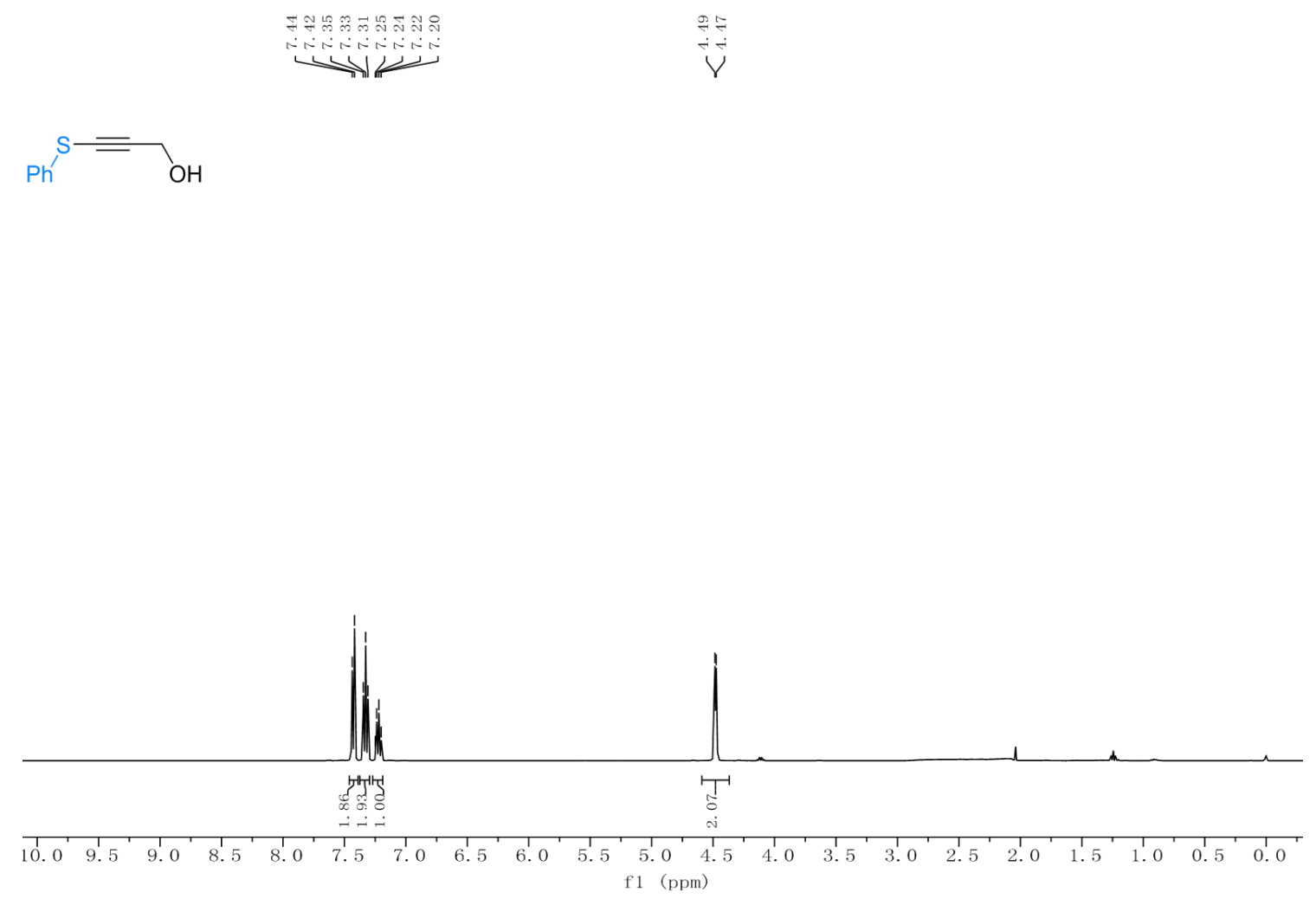

Figure S13. ${ }^{1} \mathrm{H}$ NMR spectra of $\mathbf{1 b}$. 


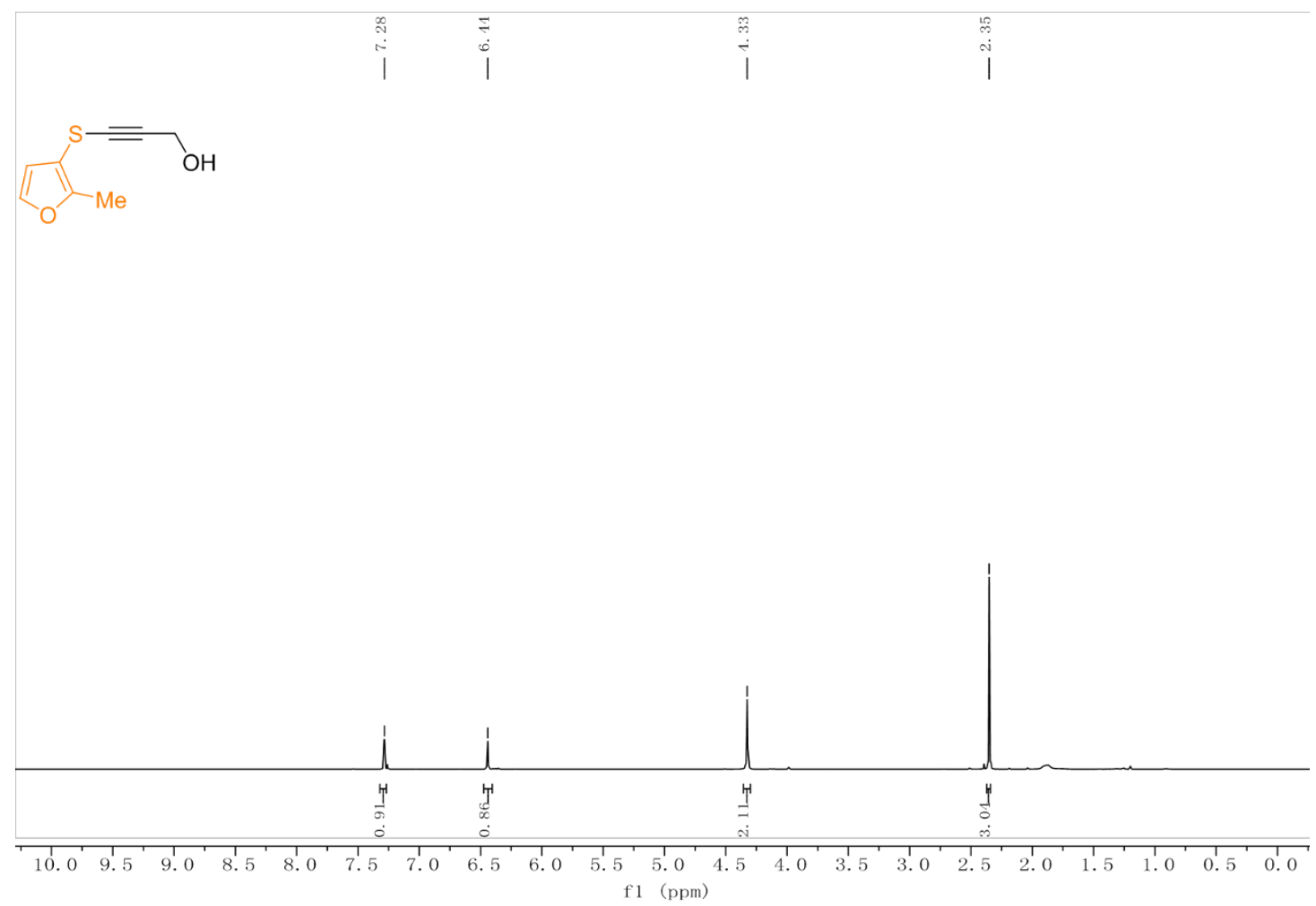

Figure S14. ${ }^{1} \mathrm{H}$ NMR spectra of 1c.<smiles>CCSC#CCO</smiles>

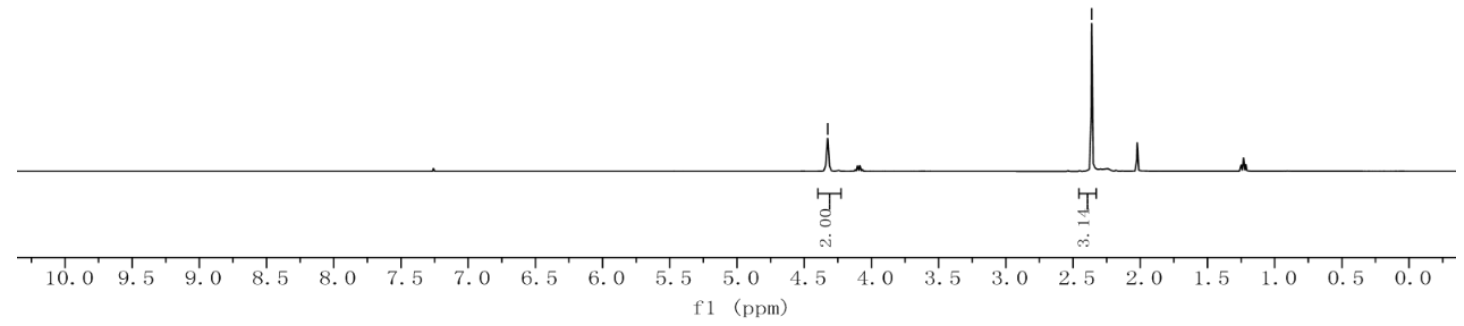

Figure S15. ${ }^{1} \mathrm{H}$ NMR spectra of $\mathbf{1 d}$. 


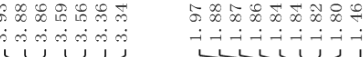

ivi

$\int_{N_{3}}^{N-B o c}$

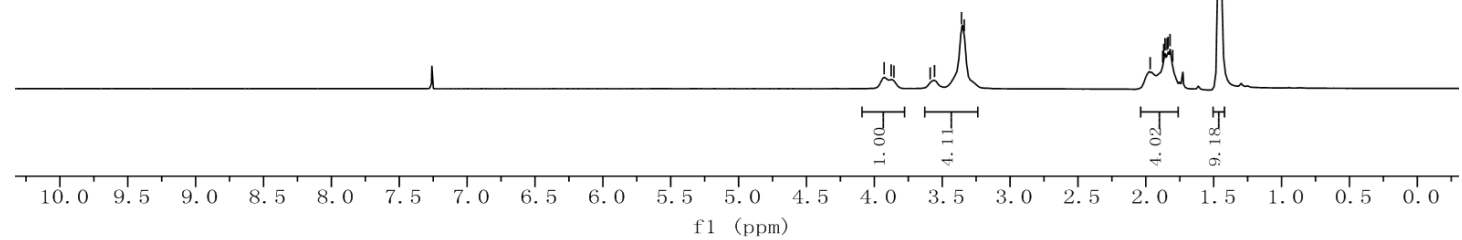

Figure S16. ${ }^{1} \mathrm{H}$ NMR spectra of 2.

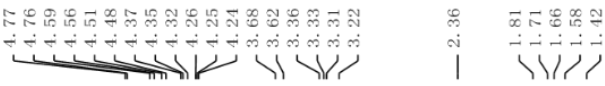<smiles>COSc1c(CO)nnn1CC1CCCN1C(=O)OC(C)(C)C</smiles>

f1 (ppm)

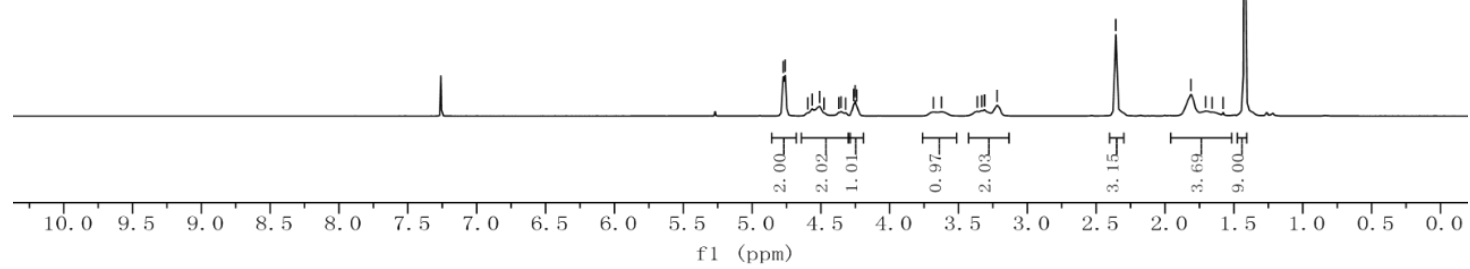

Figure S17. ${ }^{1} \mathrm{H}$ NMR spectra of $\mathbf{3 a}$. 


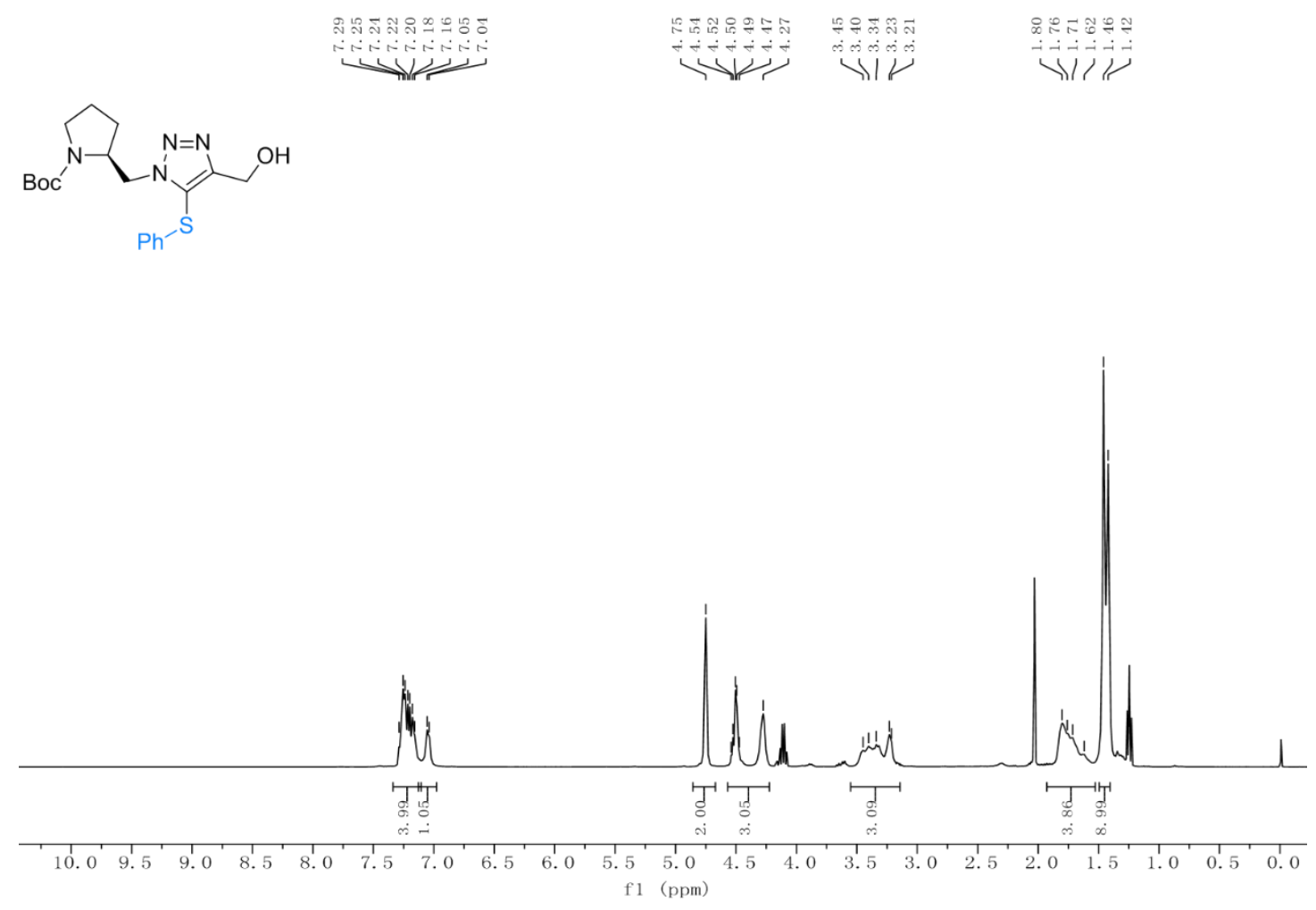

Figure S18. ${ }^{1} \mathrm{H}$ NMR spectra of $\mathbf{3 b}$.
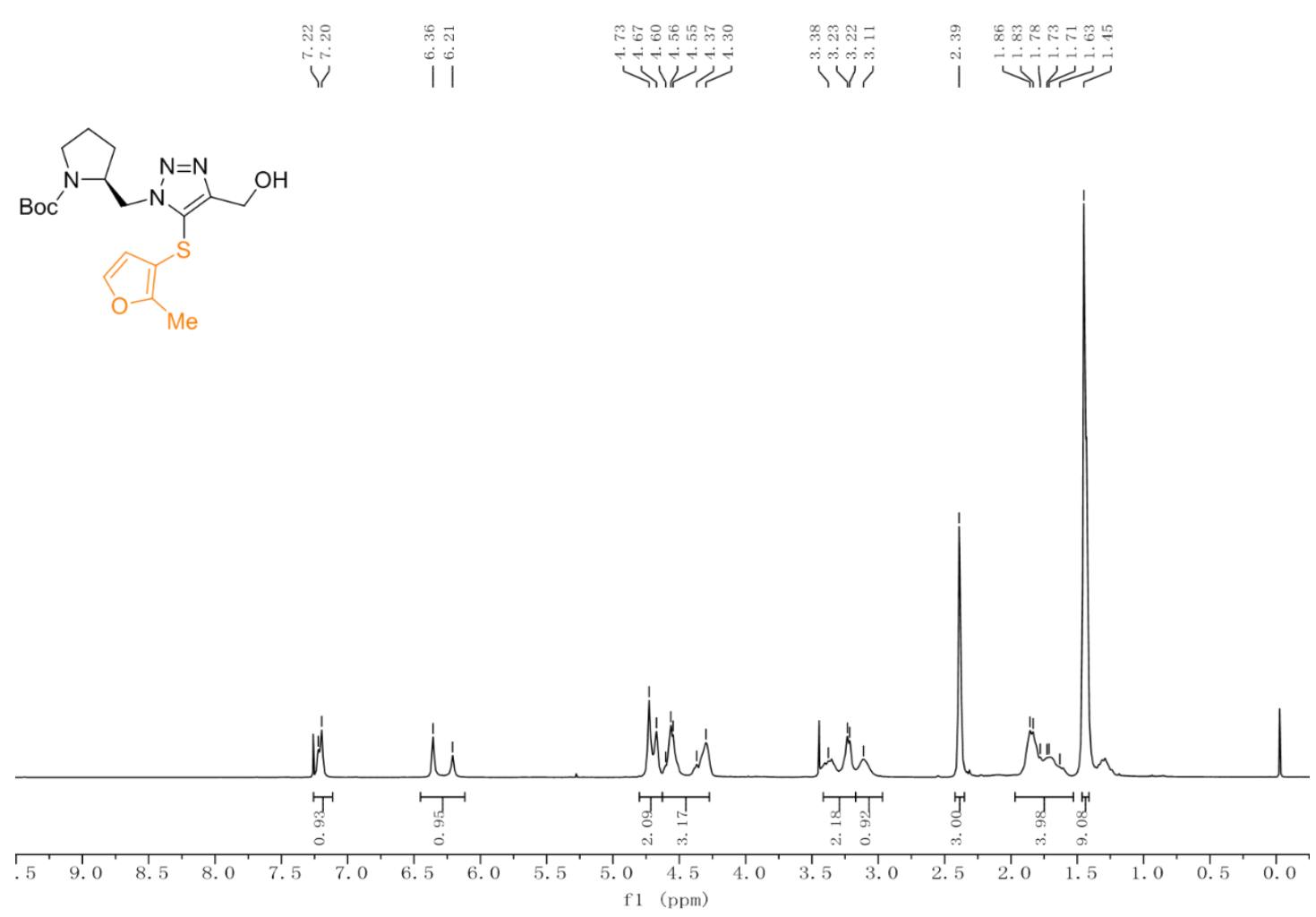

Figure S19. ${ }^{1} \mathrm{H}$ NMR spectra of $\mathbf{3 c}$. 


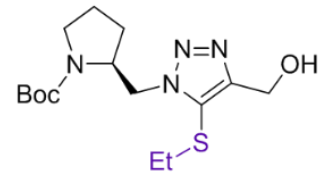

\section{(a)}

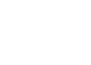




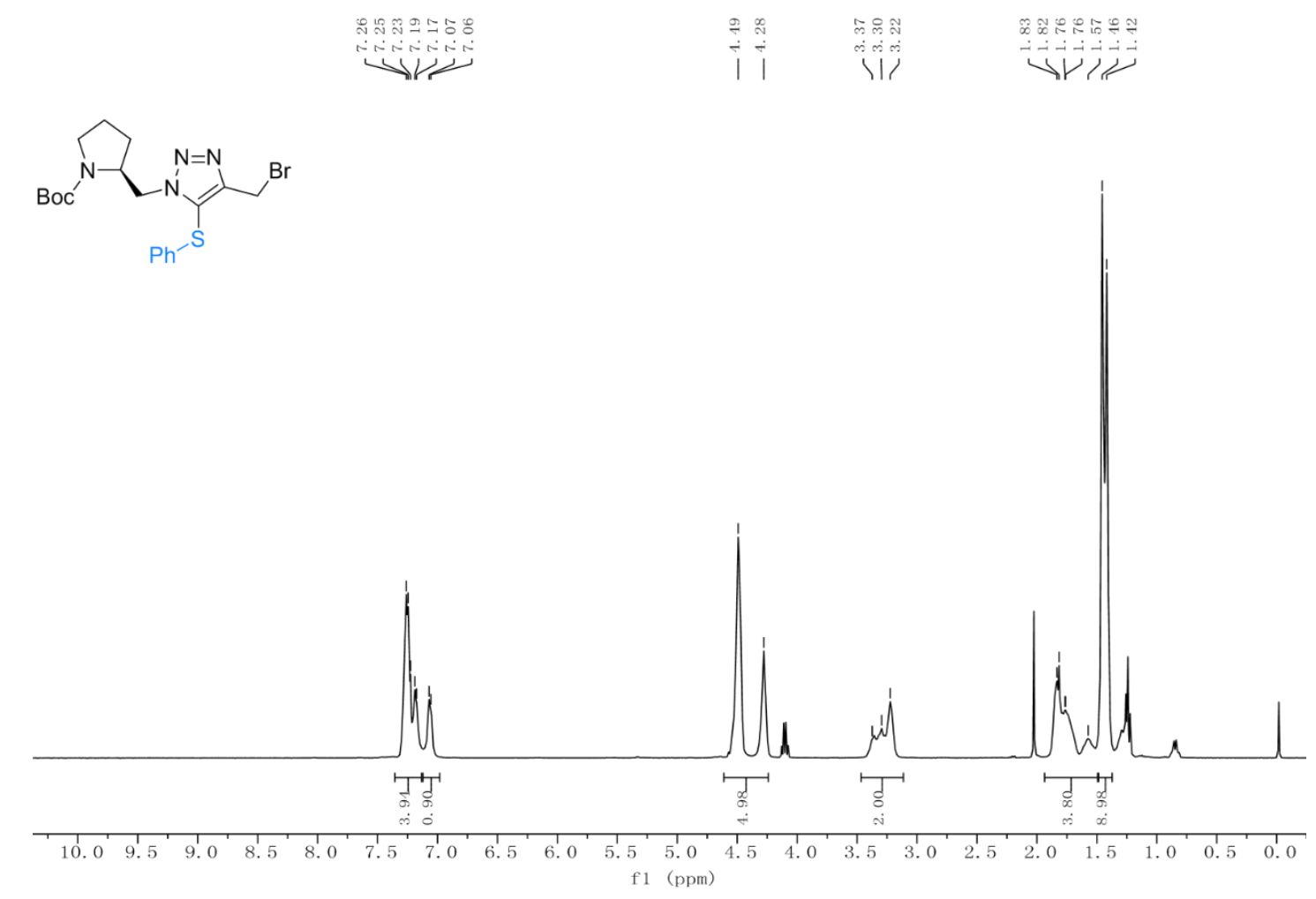

Figure S22. ${ }^{1} \mathrm{H}$ NMR spectra of $\mathbf{3 b - B r}$.

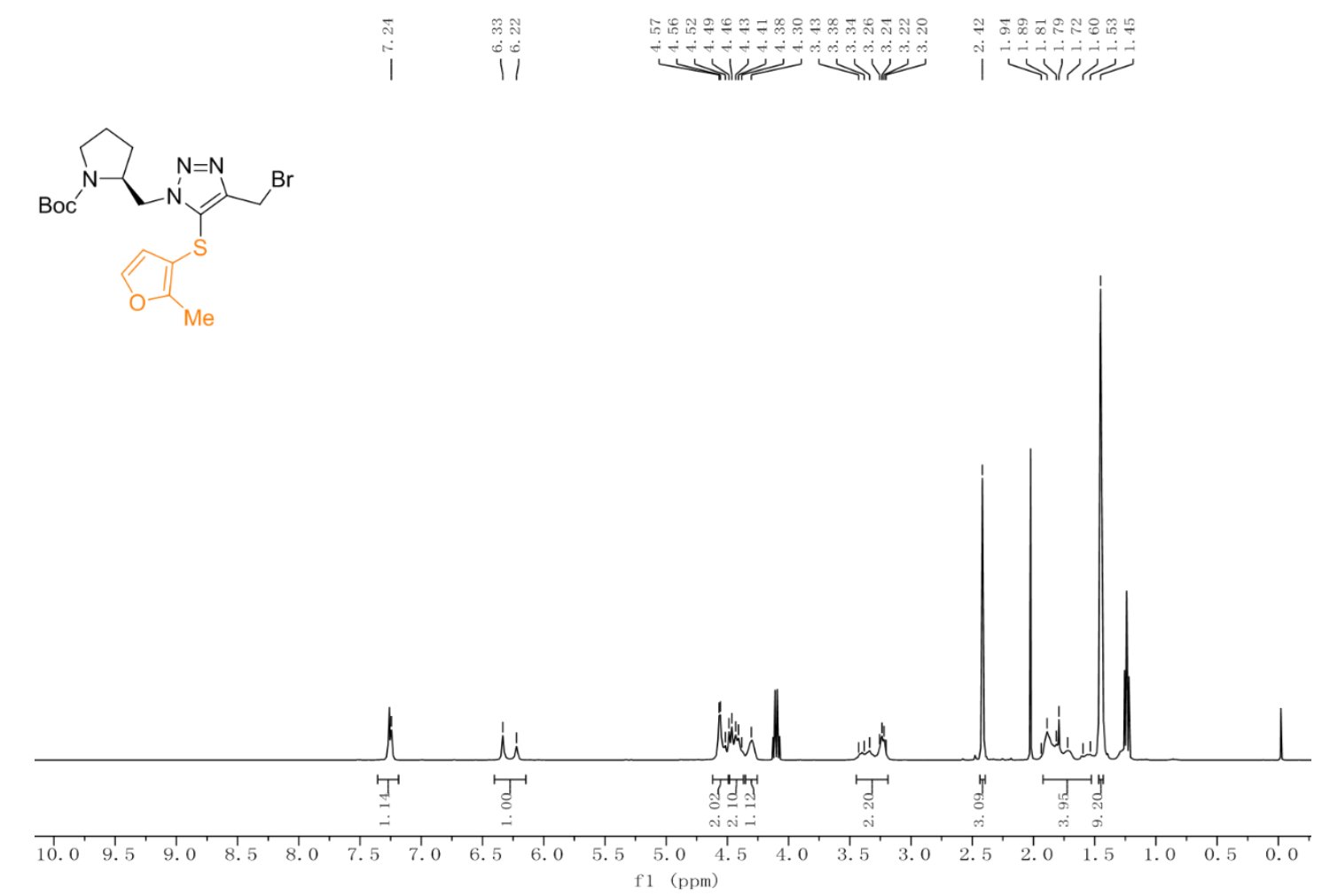

Figure S23. ${ }^{1} \mathrm{H}$ NMR spectra of 3c-Br. 

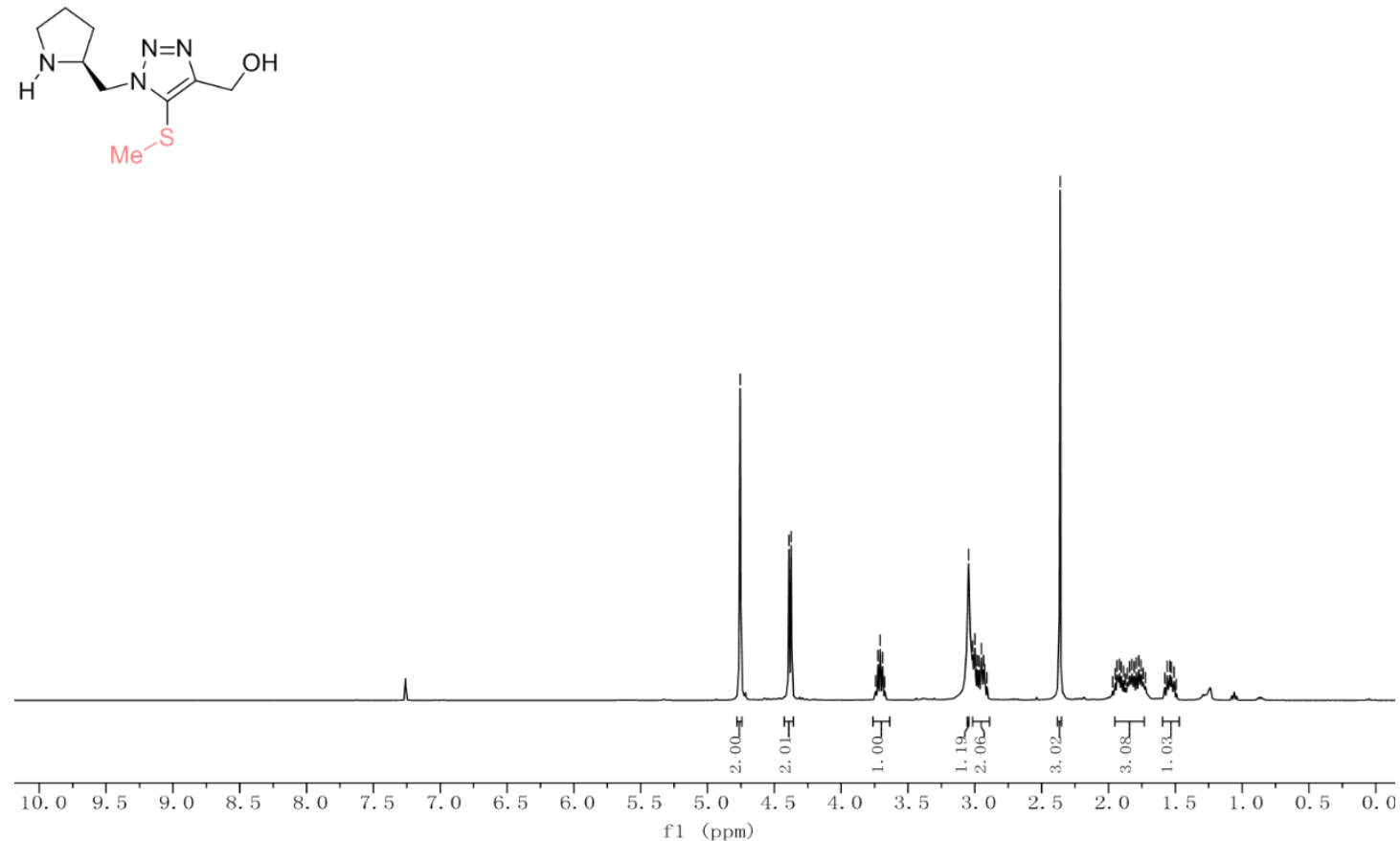

Figure S24. ${ }^{1}$ H NMR spectra of 3a-H.

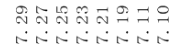

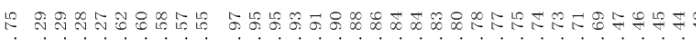
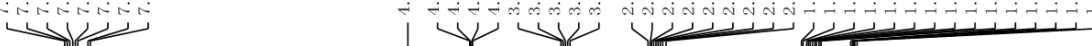<smiles>OCc1nnc(SCc2ccccc2)n1CC1CCCN1</smiles>

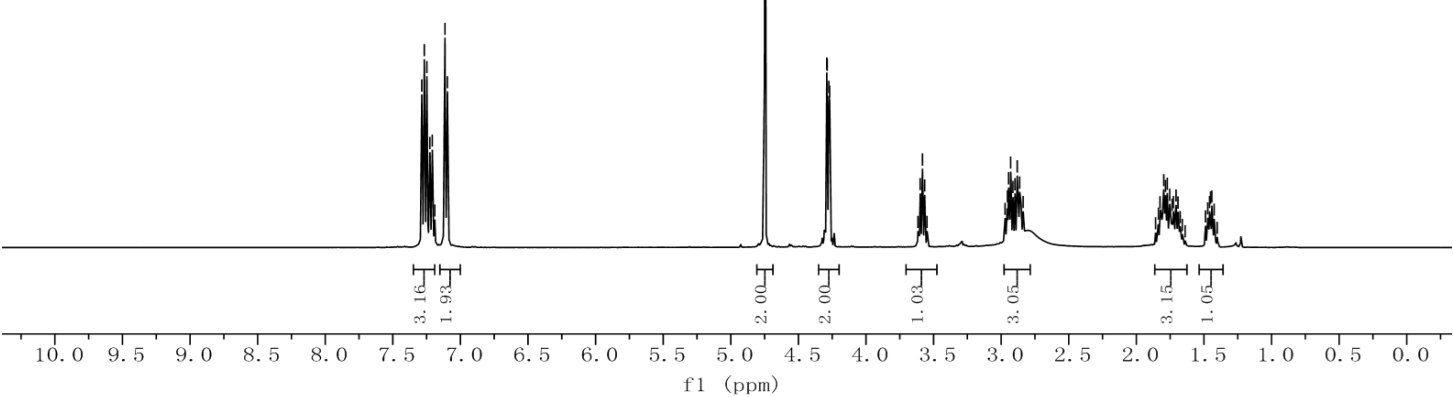

Figure S25. ${ }^{1} \mathrm{H}$ NMR spectra of $\mathbf{3 b}-\mathbf{H}$. 

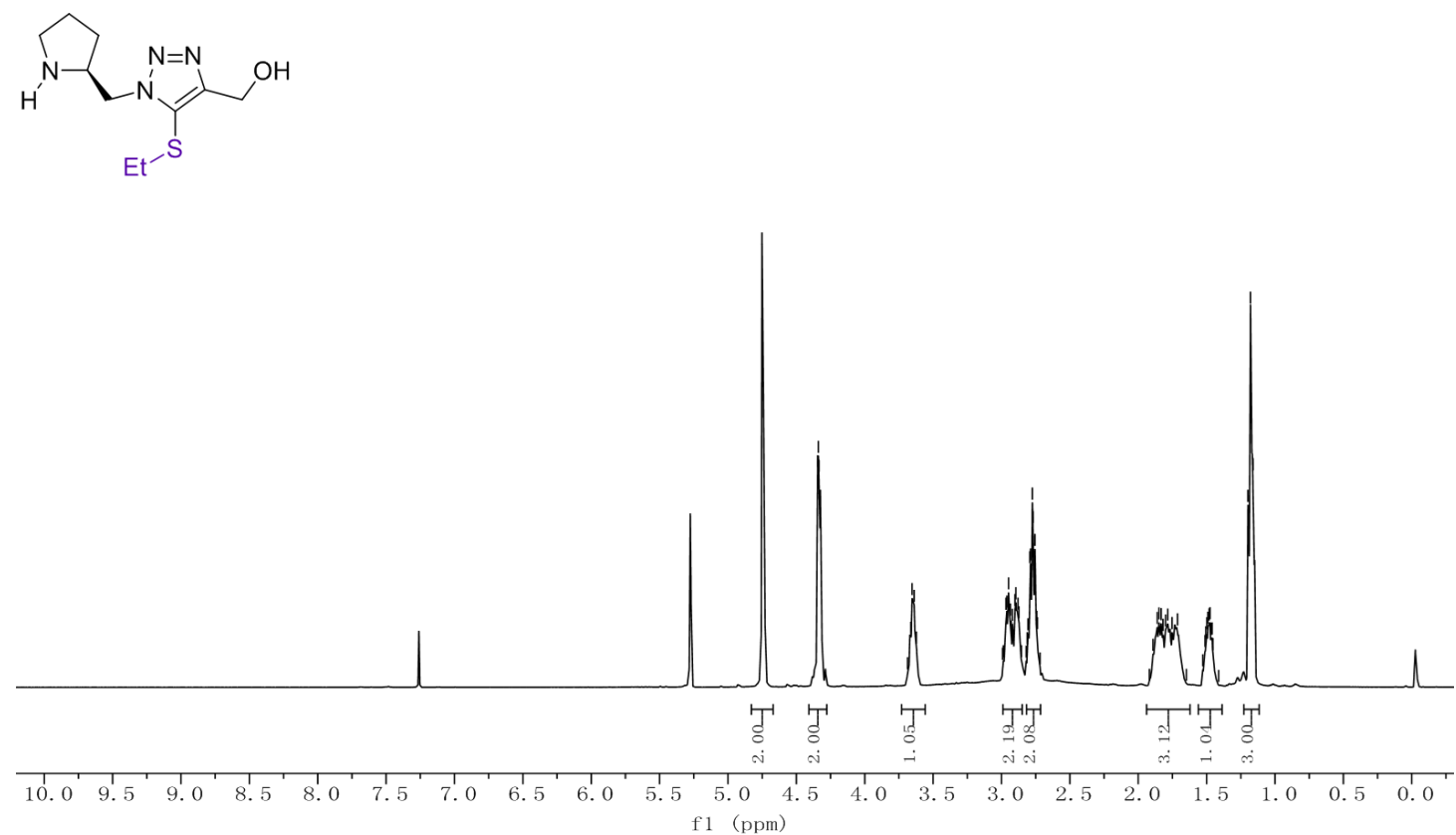

Figure S26. ${ }^{1} \mathrm{H}$ NMR spectra of $\mathbf{3 d - H}$.

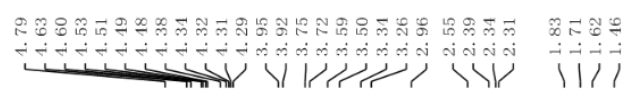<smiles>CSc1c(CN2CCCC2Cn2nnc(CO)c2S(C)(=O)=O)nnn1CC1CCCN1C(=O)OC(C)(C)C</smiles>

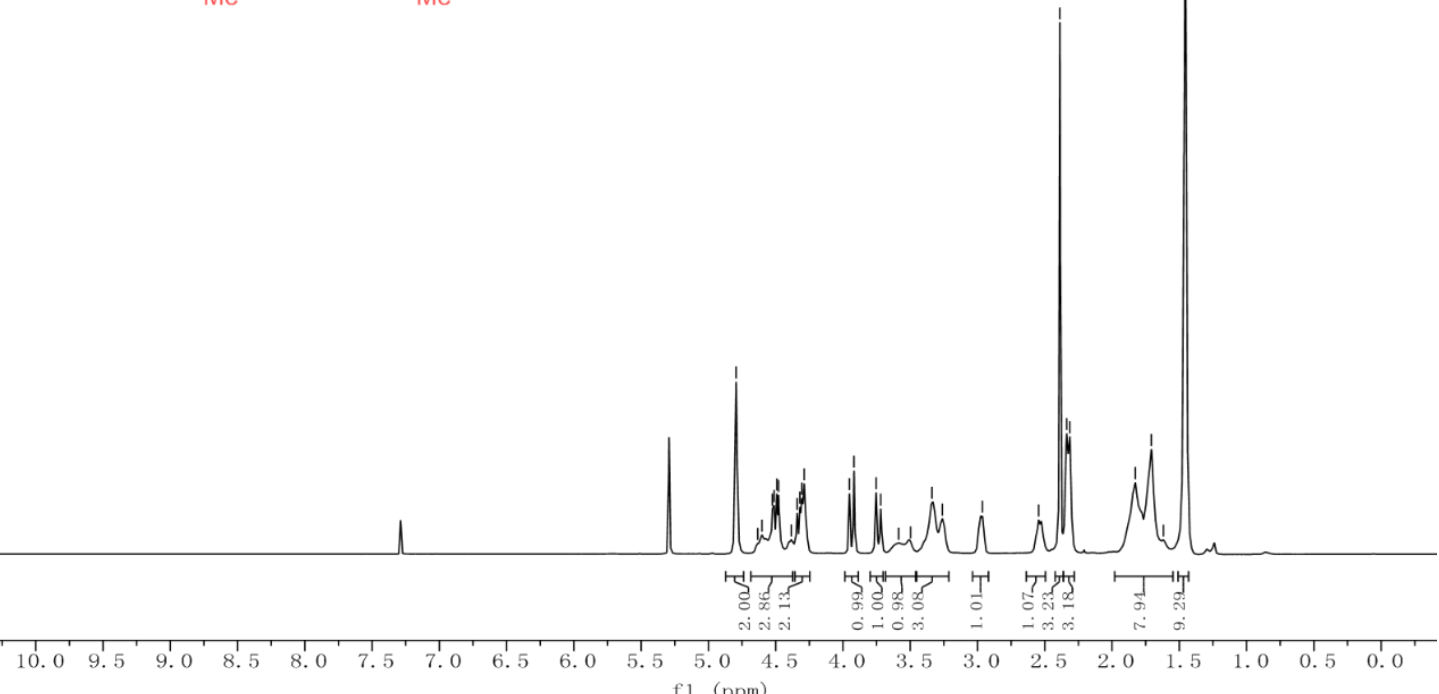

Figure S27. ${ }^{1} \mathrm{H}$ NMR spectra of $\mathbf{4 a}$. 

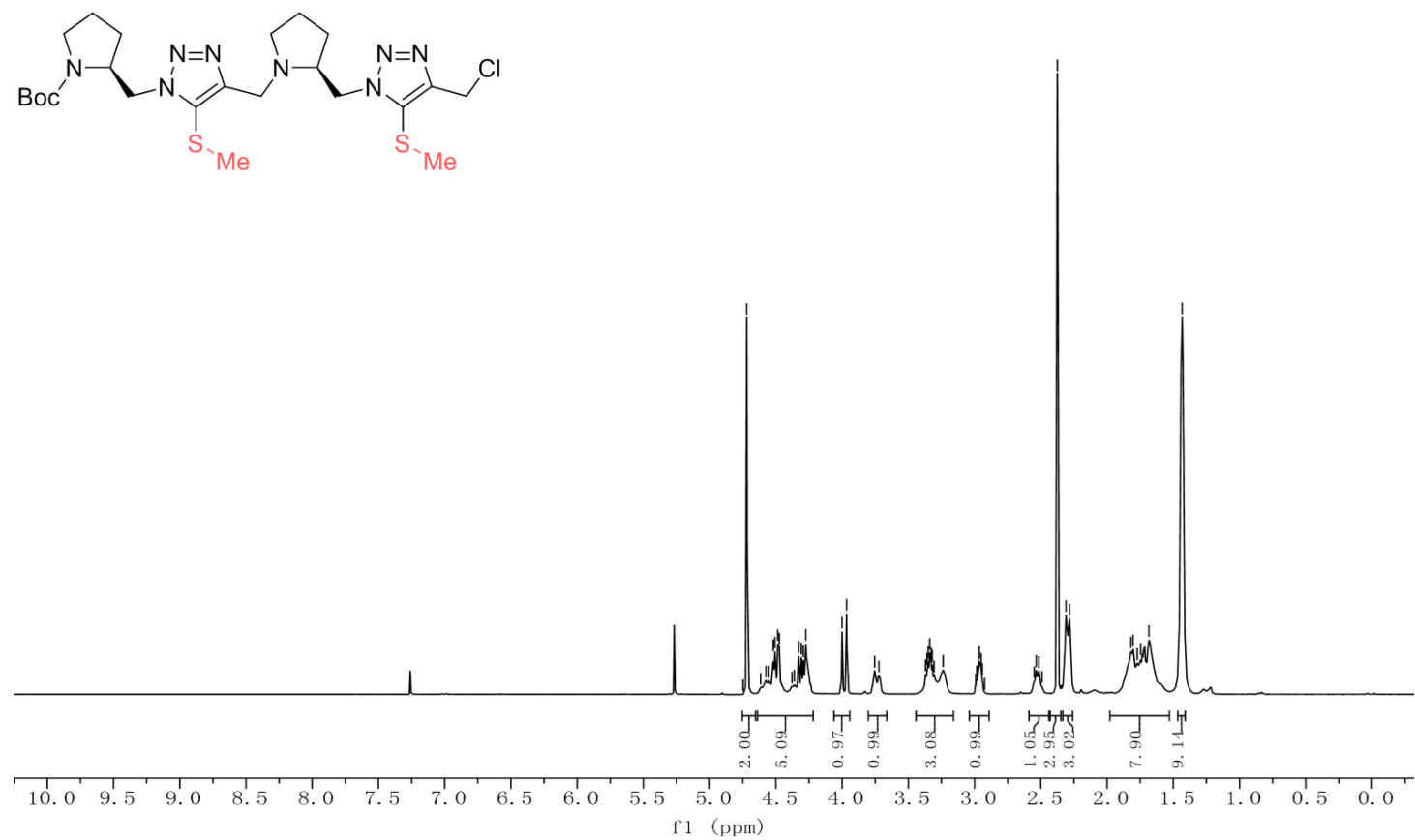

Figure S28. ${ }^{1} \mathrm{H}$ NMR spectra of $\mathbf{4 a - C l}$.
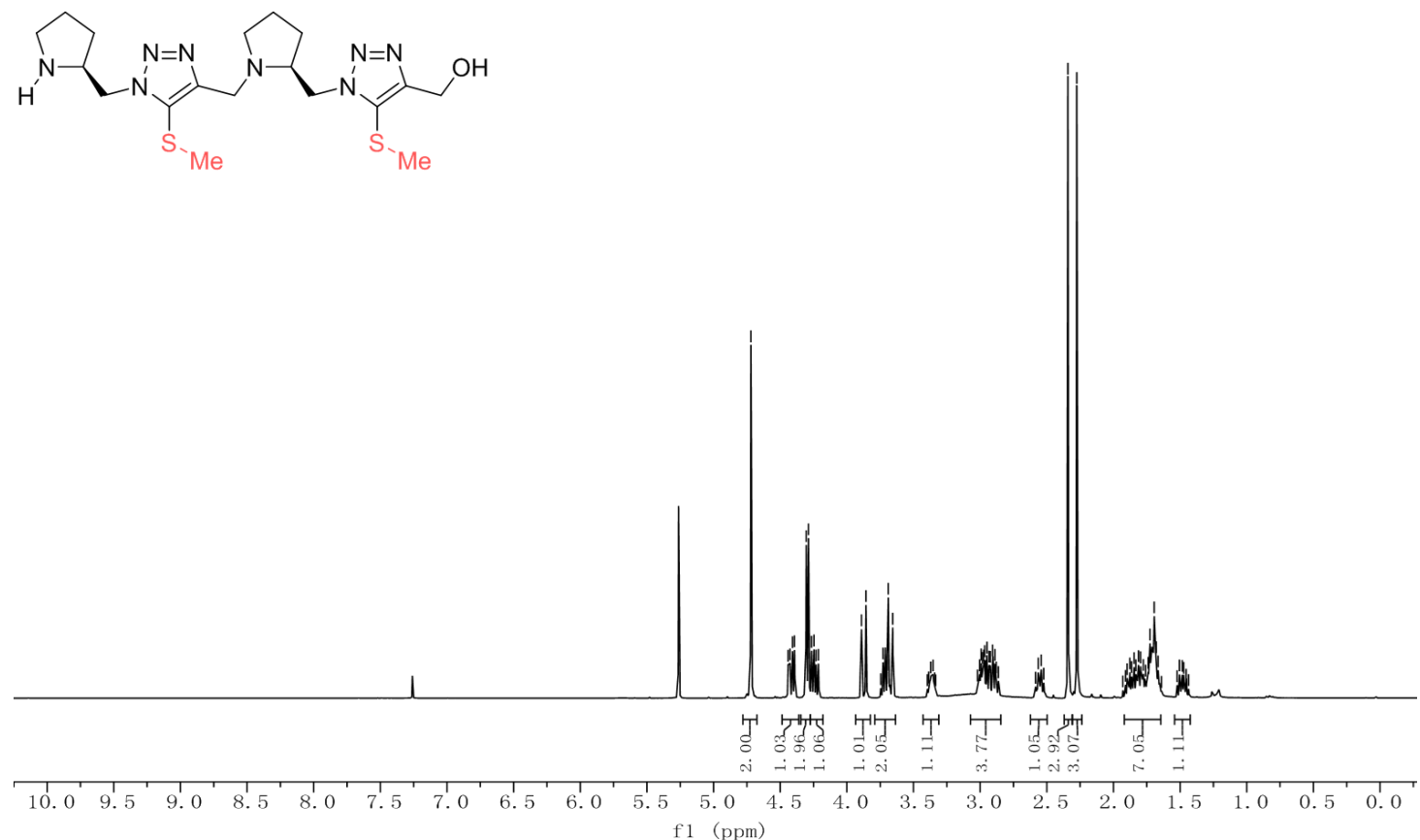

Figure S29. ${ }^{1} \mathrm{H}$ NMR spectra of $\mathbf{4 a - H}$. 

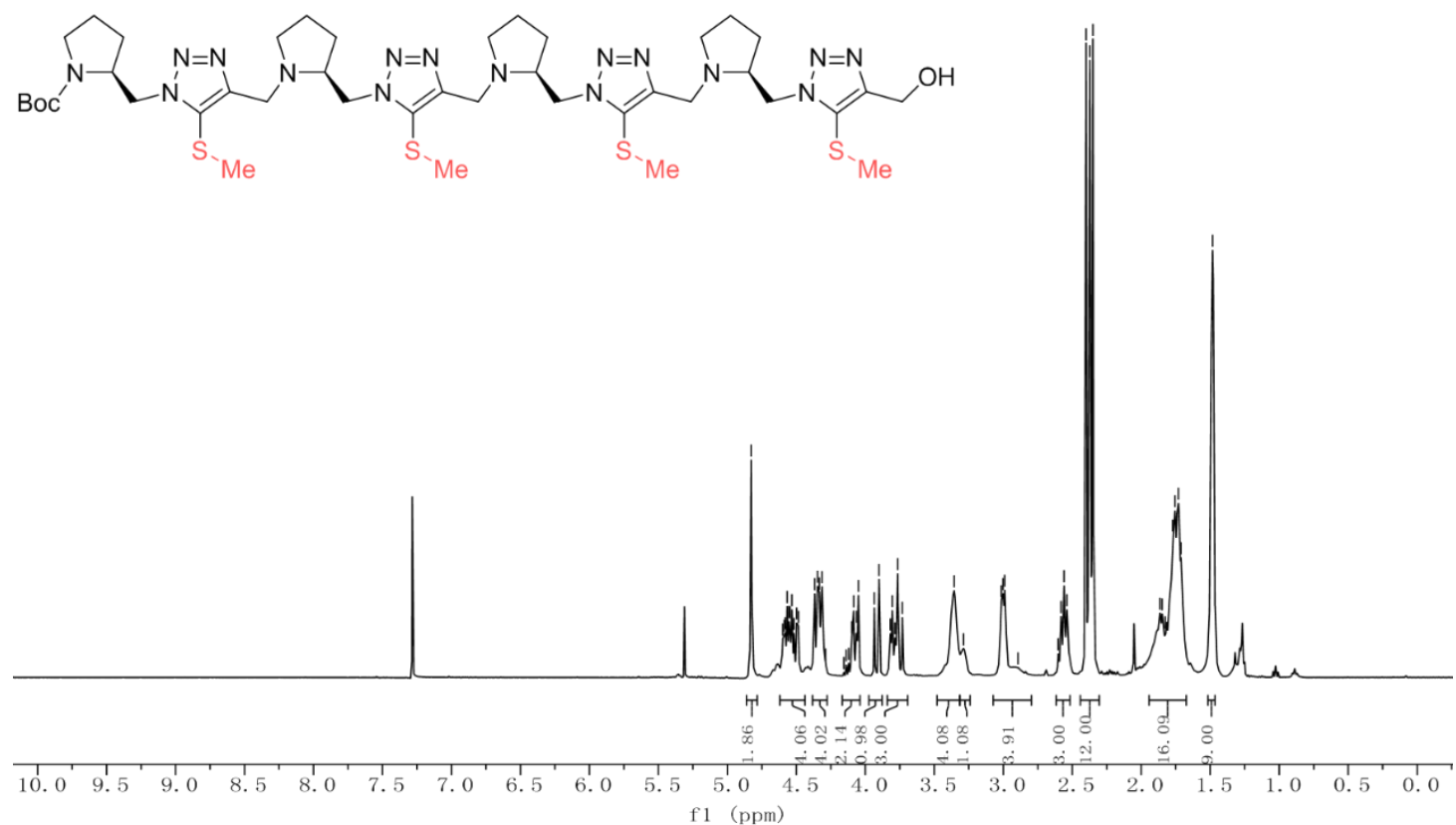

Figure S30. ${ }^{1} \mathrm{H}$ NMR spectra of $\mathbf{6 a}$.<smiles></smiles>

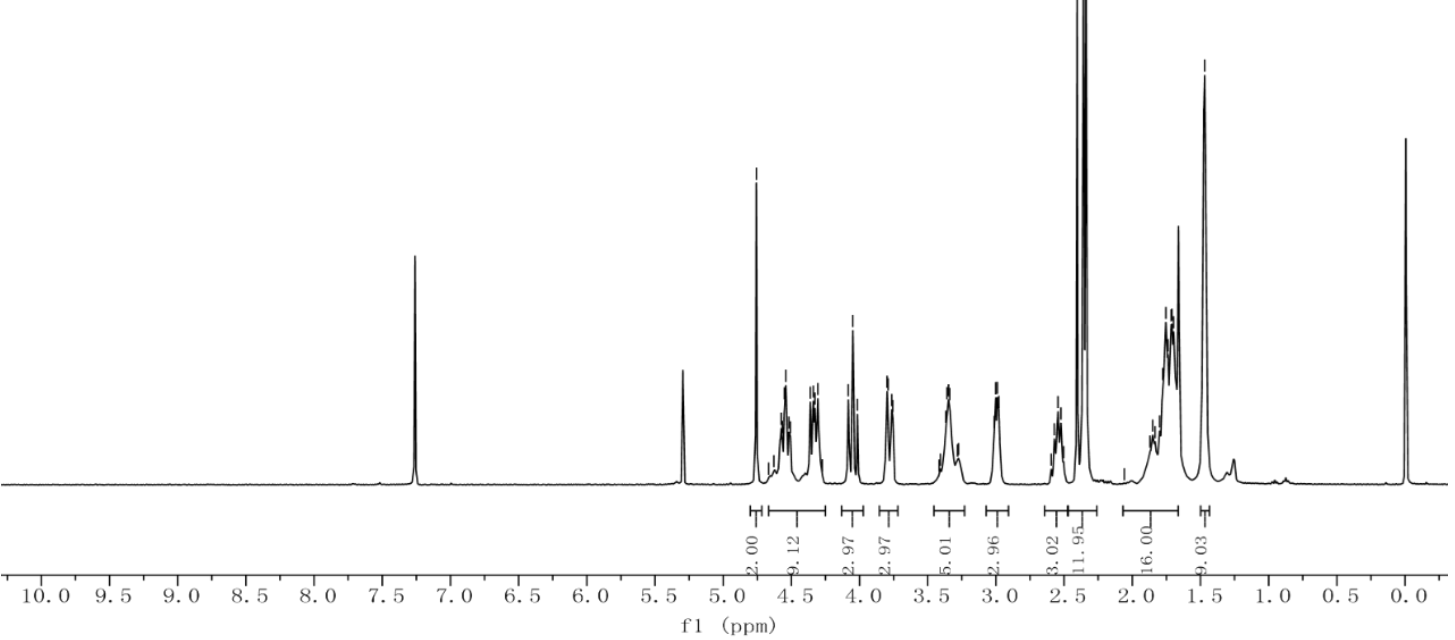

Figure S31. ${ }^{1} \mathrm{H}$ NMR spectra of 6a-Cl. 

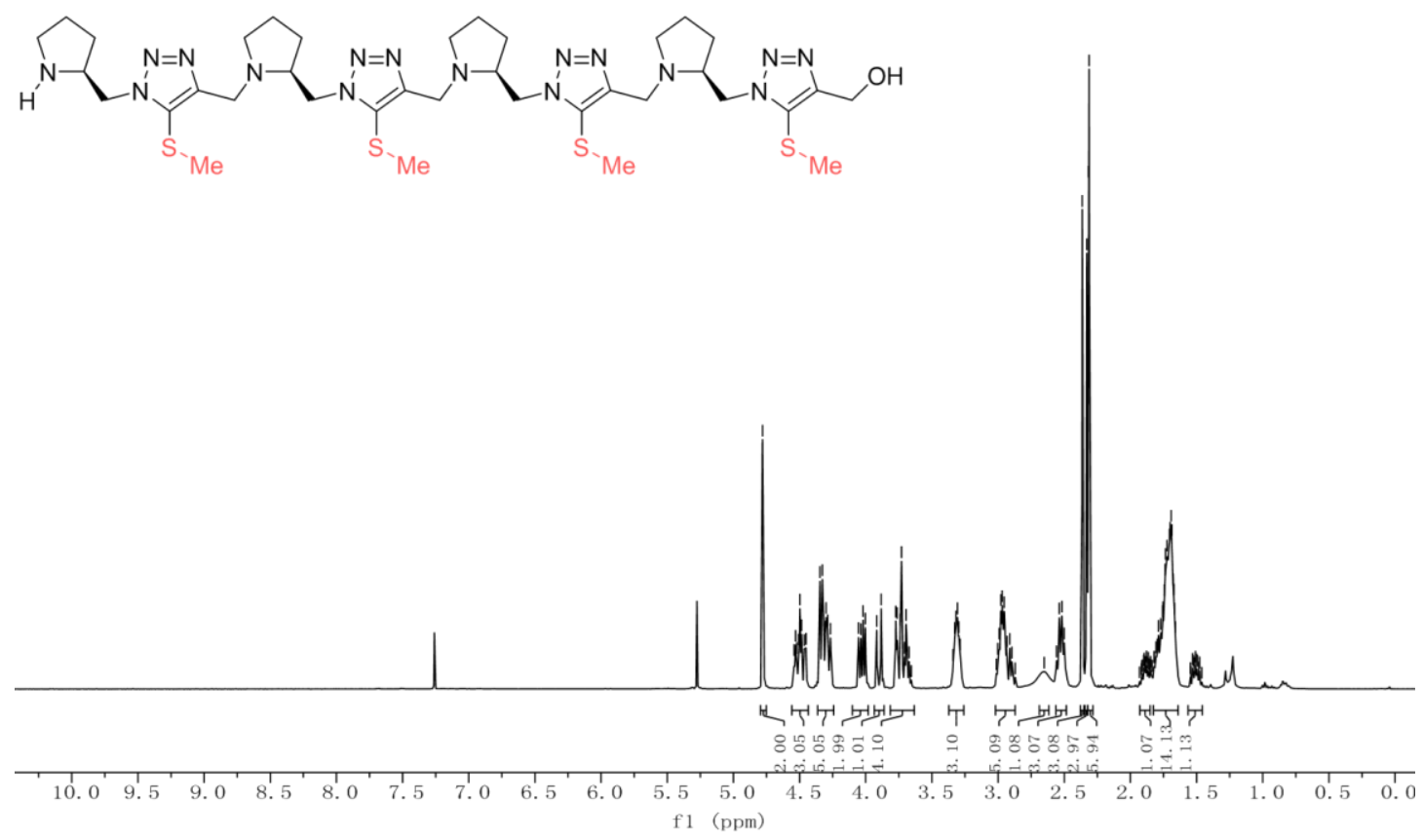

Figure S32. ${ }^{1} \mathrm{H}$ NMR spectra of $\mathbf{6 a - H}$.

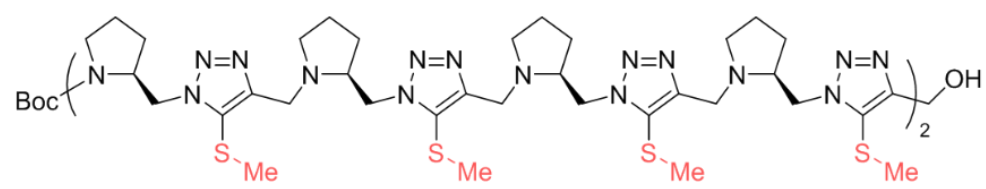


Boc

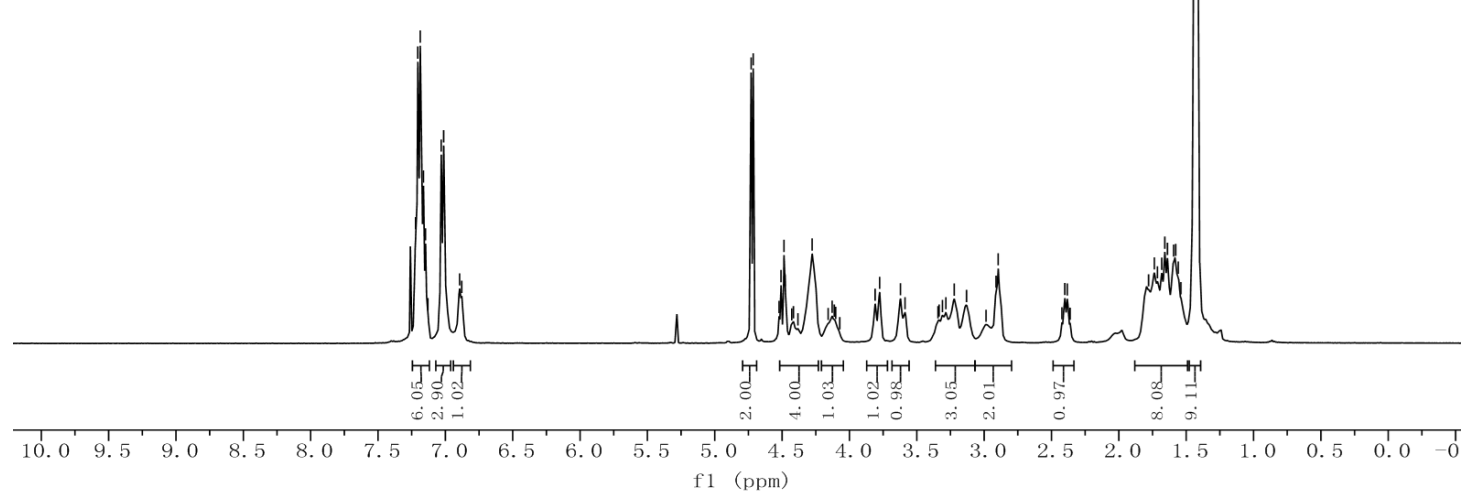

Figure S34. ${ }^{1} \mathrm{H}$ NMR spectra of $\mathbf{4 b}$.

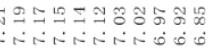<smiles>CC(C)(C)OC(=O)N1CCCC1Cn1nnc(CN2CCCC2Cn2nnc(CBr)c2Sc2ccccc2)c1Sc1ccccc1</smiles>

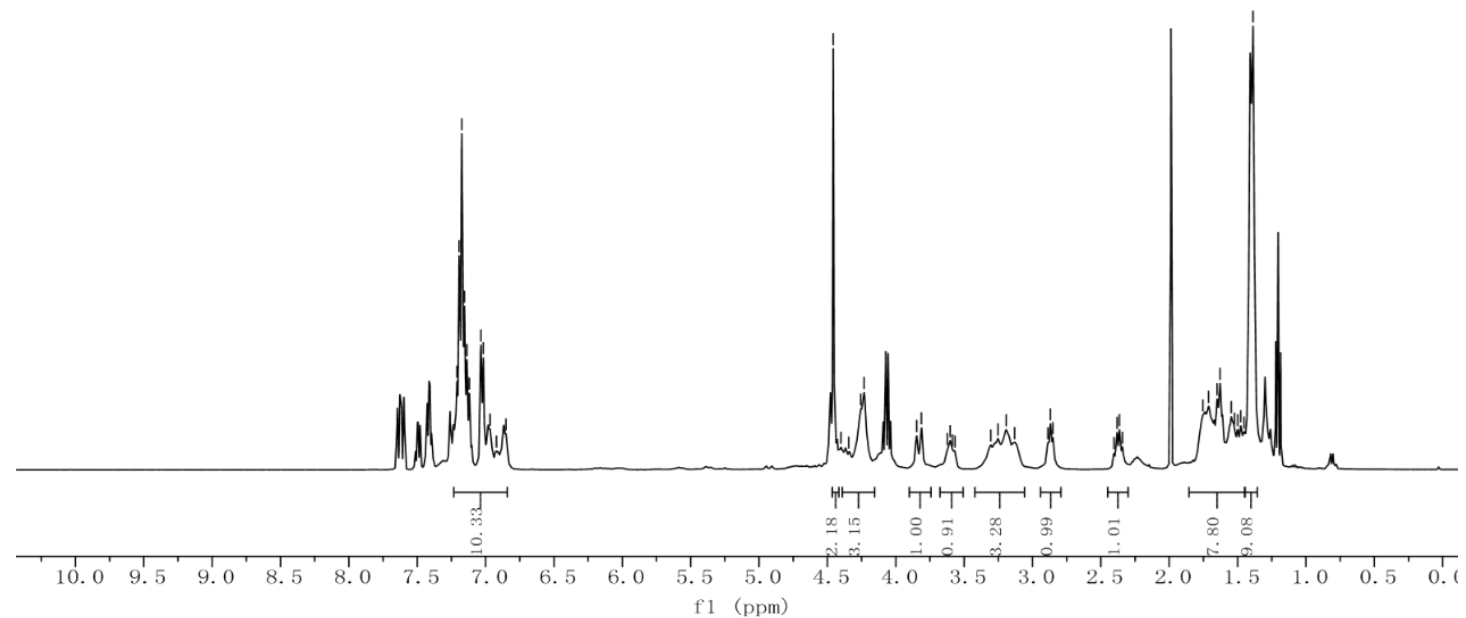

Figure S35. ${ }^{1} \mathrm{H}$ NMR spectra of $\mathbf{4 b - B r}$. 
$\overbrace{S_{P h}}^{N=N}$

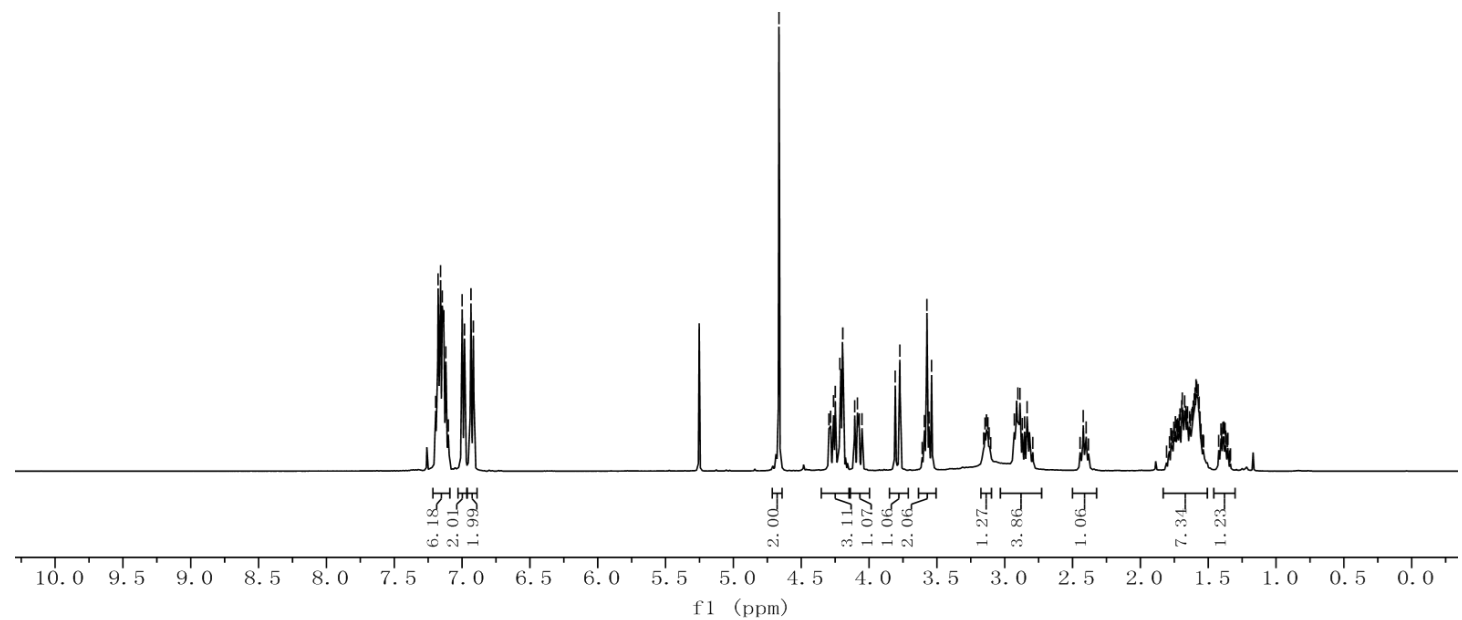

Figure S36. ${ }^{1} \mathrm{H}$ NMR spectra of $\mathbf{4 b - H}$.<smiles>CC(C)(C)OC(=O)N1CCC[C@H]1Cn1cc(CN2CCC[C@H]2Cn2nnc(CN3CCC[C@H]3Cn3nnc(CN4CCC[C@H]4Cn4nnc(CO)c4Sc4ccccc4)c3SPc3ccccc3)c2Sc2ccccc2)nn1</smiles>

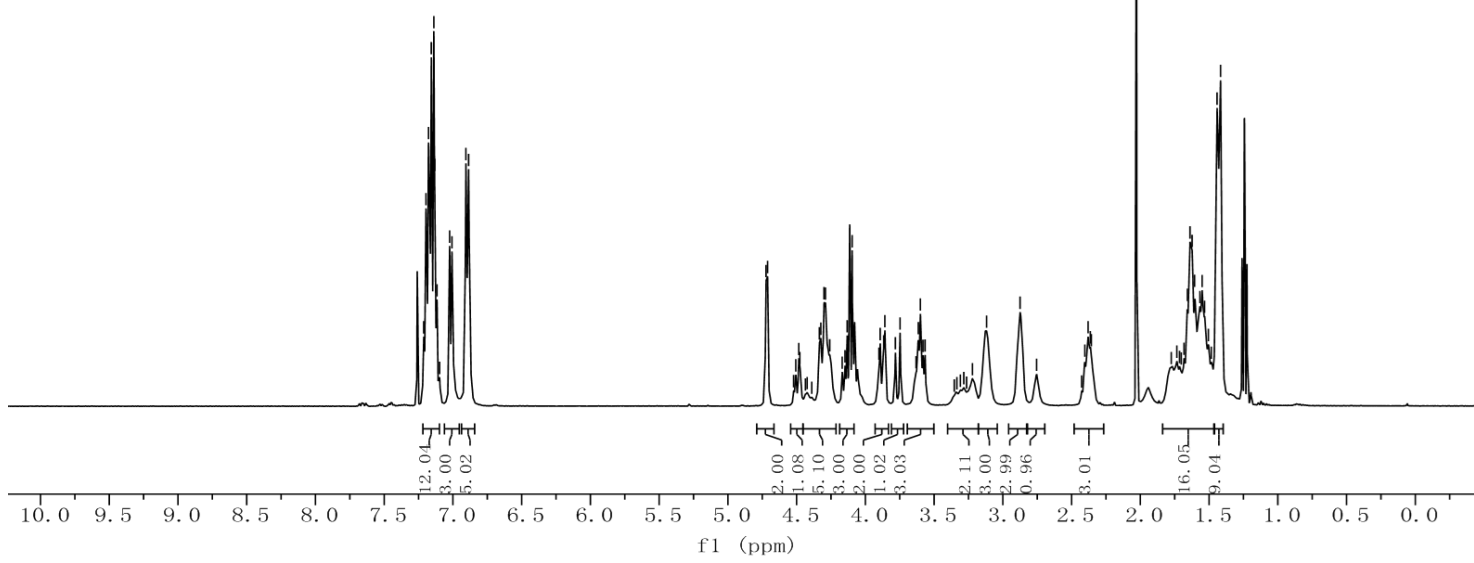

Figure S37 ${ }^{1} \mathrm{H}$ NMR spectra of $\mathbf{6 b}$. 
<smiles>O=C(c1ccccc1)N1CCCC1Cn1cc(CN2CCCC2Cn2nnc(CN3CCCC3Cn3nnc(CN4CCCC4Cn4nnc(CBr)c4Sc4ccccc4)c3Sc3ccccc3)c2Sc2ccccc2)nn1</smiles>

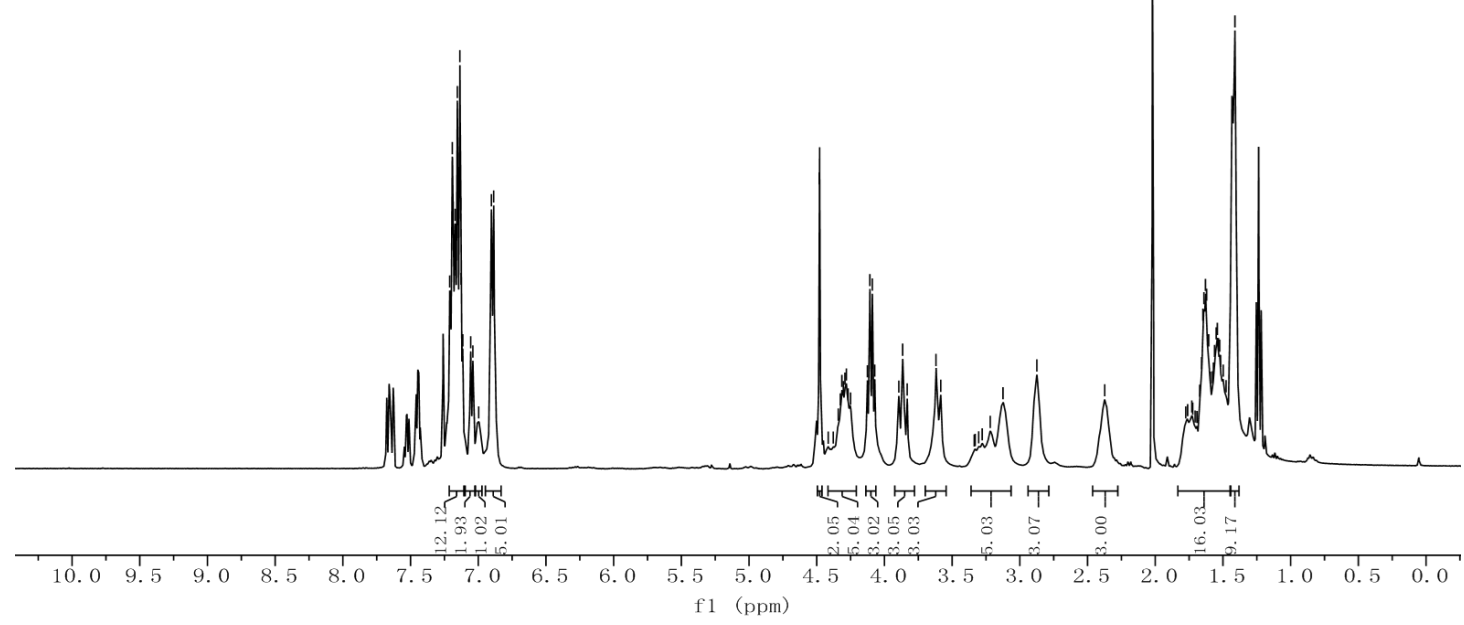

Figure S38. ${ }^{1} \mathrm{H}$ NMR spectra of $6 \mathbf{b}-\mathbf{B r}$.

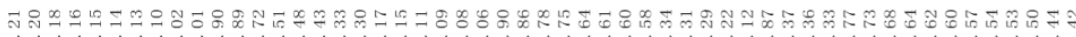

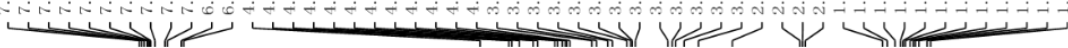<smiles>CC(C)(C)OC(=O)N1CCCC1Cn1nnc(CN2CCCC2Cn2nnc(CN3CCCC3Cn3nnc(CN4CCCC4Cn4nnc(CO)c4Sc4ccccc4)c3Sc3ccccc3)c2Sc2ccccc2)c1Sc1ccccc1</smiles>

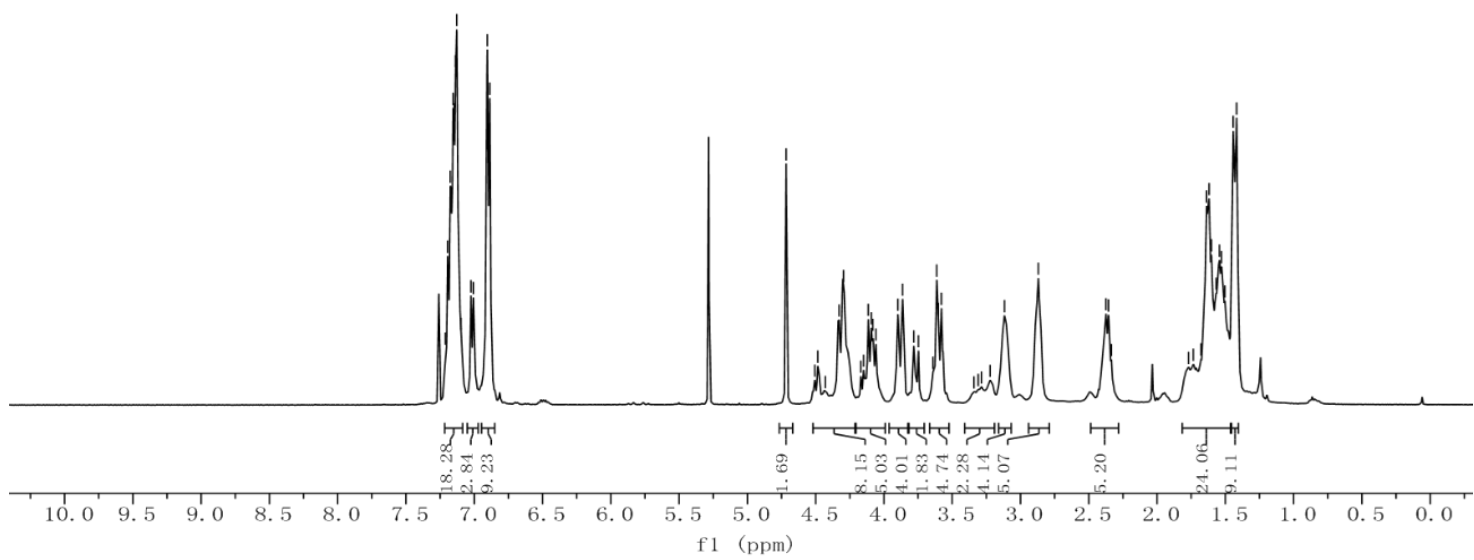

Figure S39. ${ }^{1} \mathrm{H}$ NMR spectra of $\mathbf{7 b}$. 


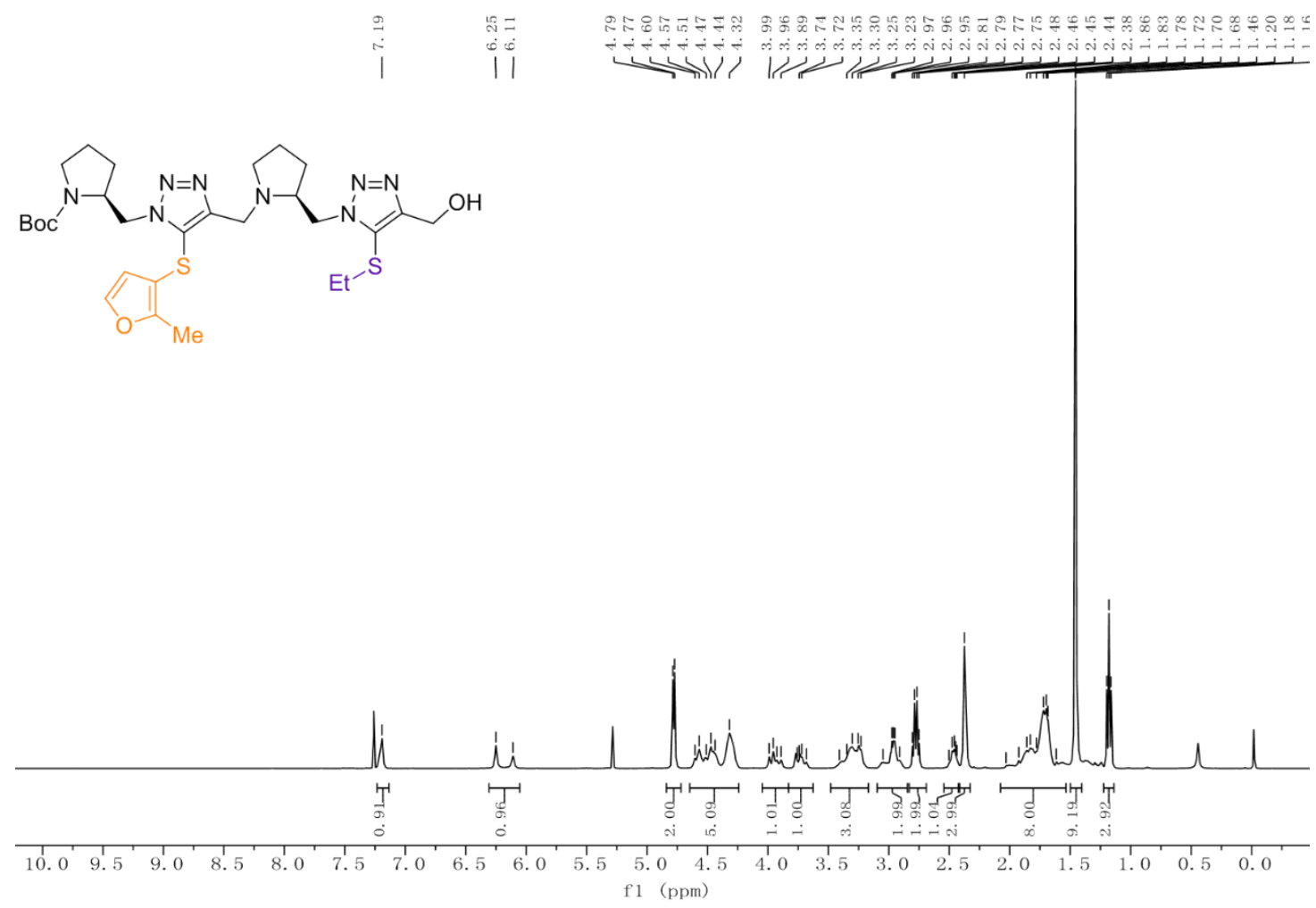

Figure S40. ${ }^{1} \mathrm{H}$ NMR spectra of $\mathbf{4 c}$.
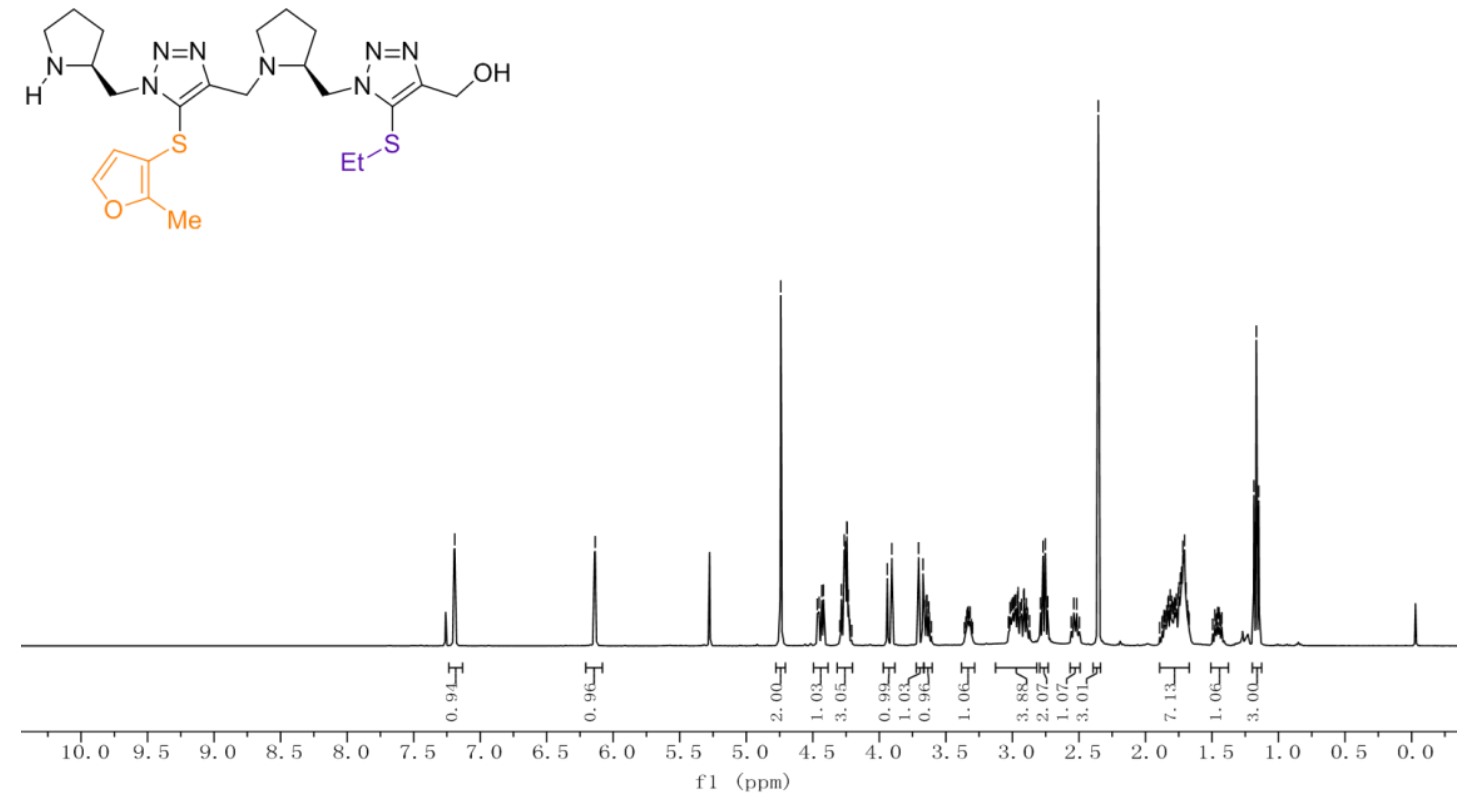

Figure S41. ${ }^{1} \mathrm{H}$ NMR spectra of $\mathbf{4 c - H}$. 


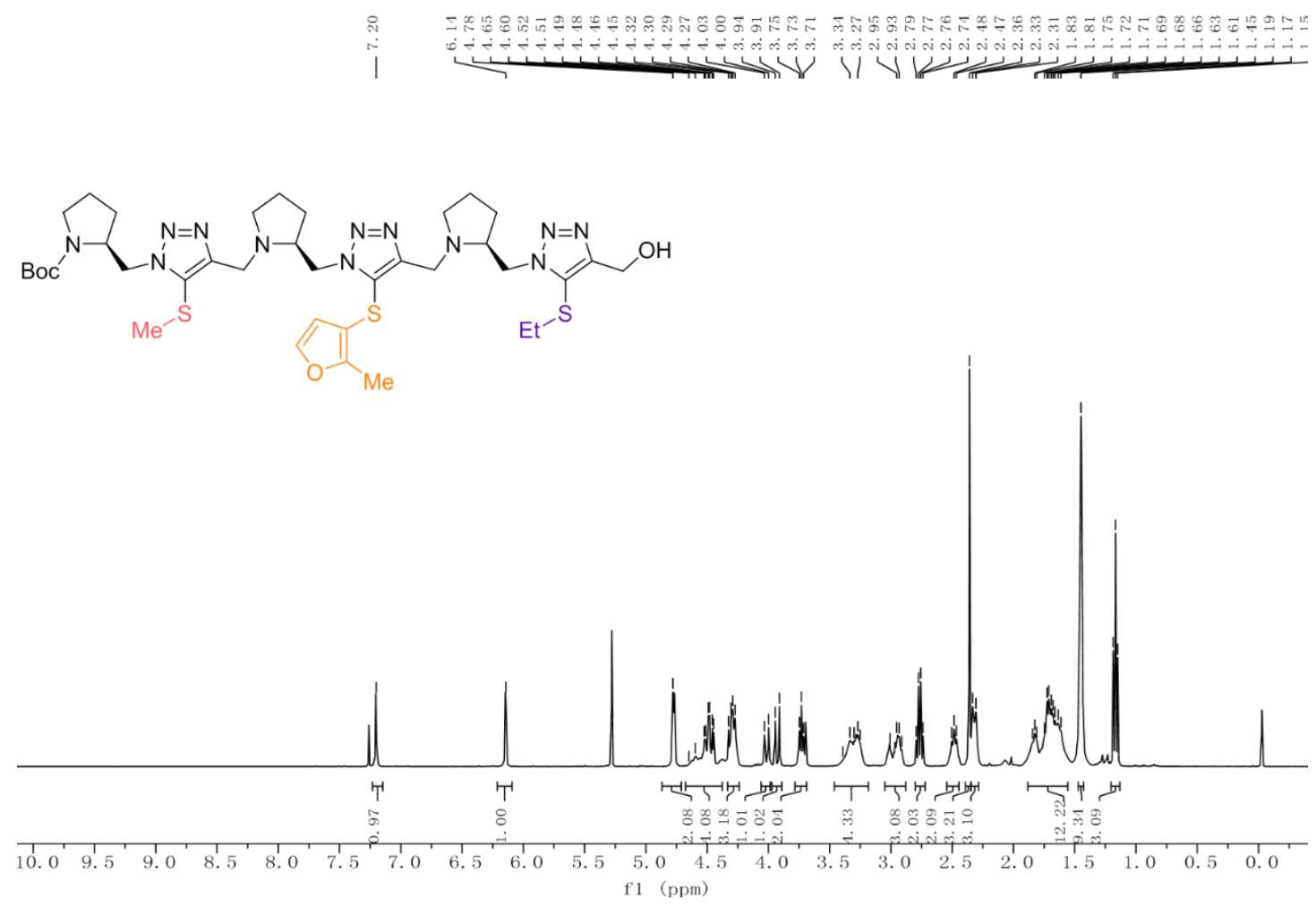

Figure S42. ${ }^{1} \mathrm{H}$ NMR spectra of $\mathbf{5 c}$.
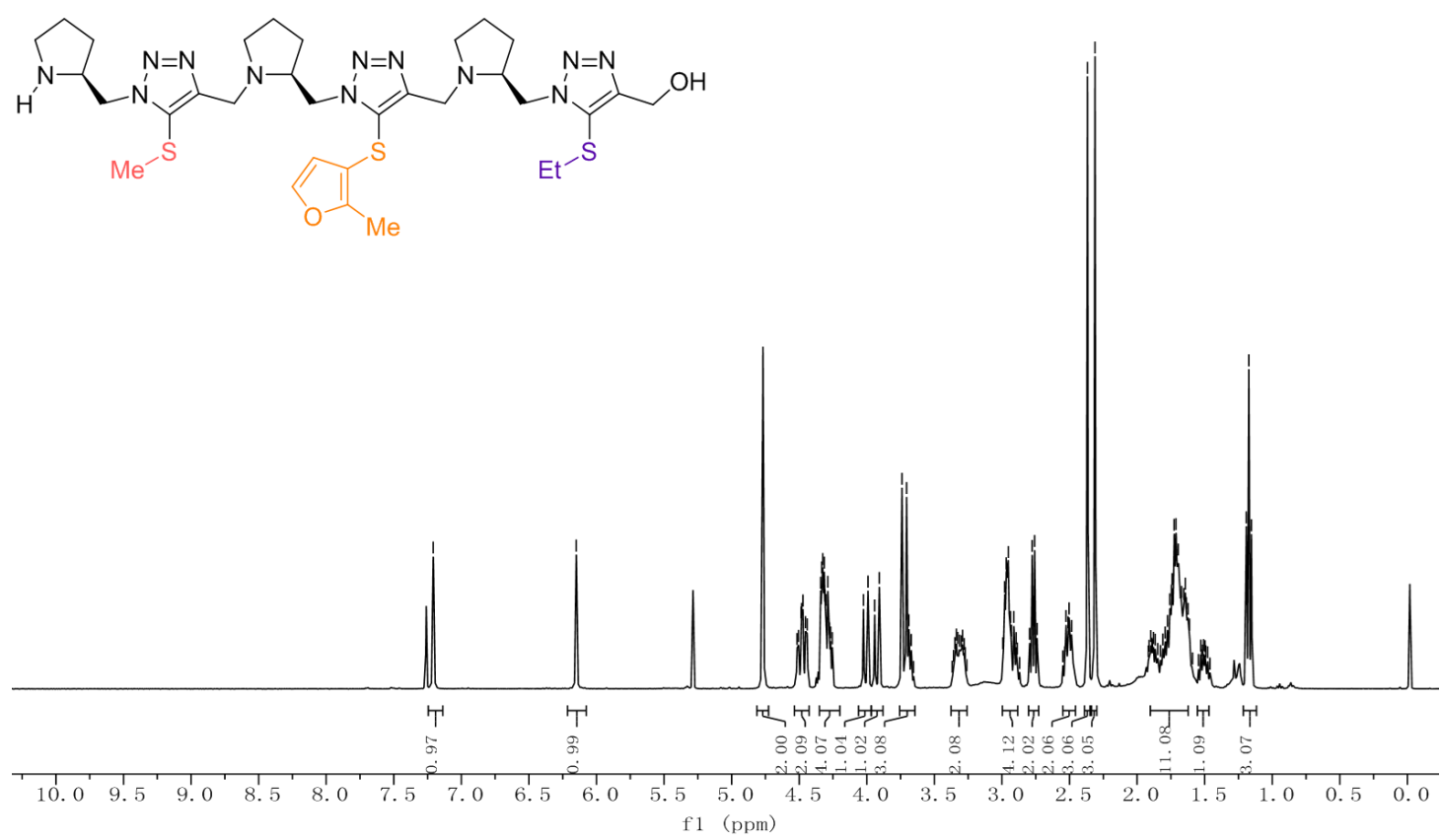

Figure S43. ${ }^{1} \mathrm{H}$ NMR spectra of $\mathbf{5 c - H}$. 

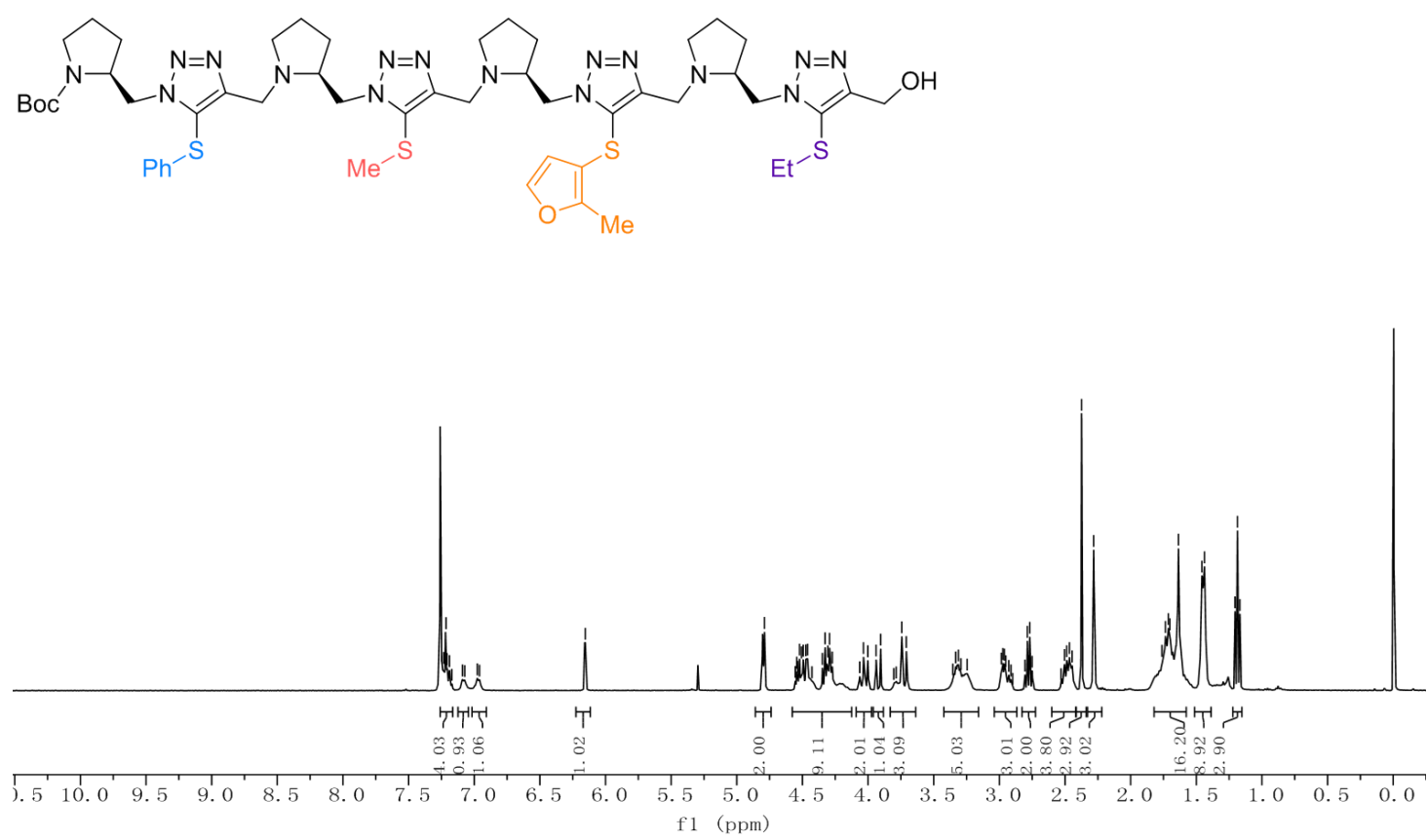

Figure S44. ${ }^{1} \mathrm{H}$ NMR spectra of $6 c$.

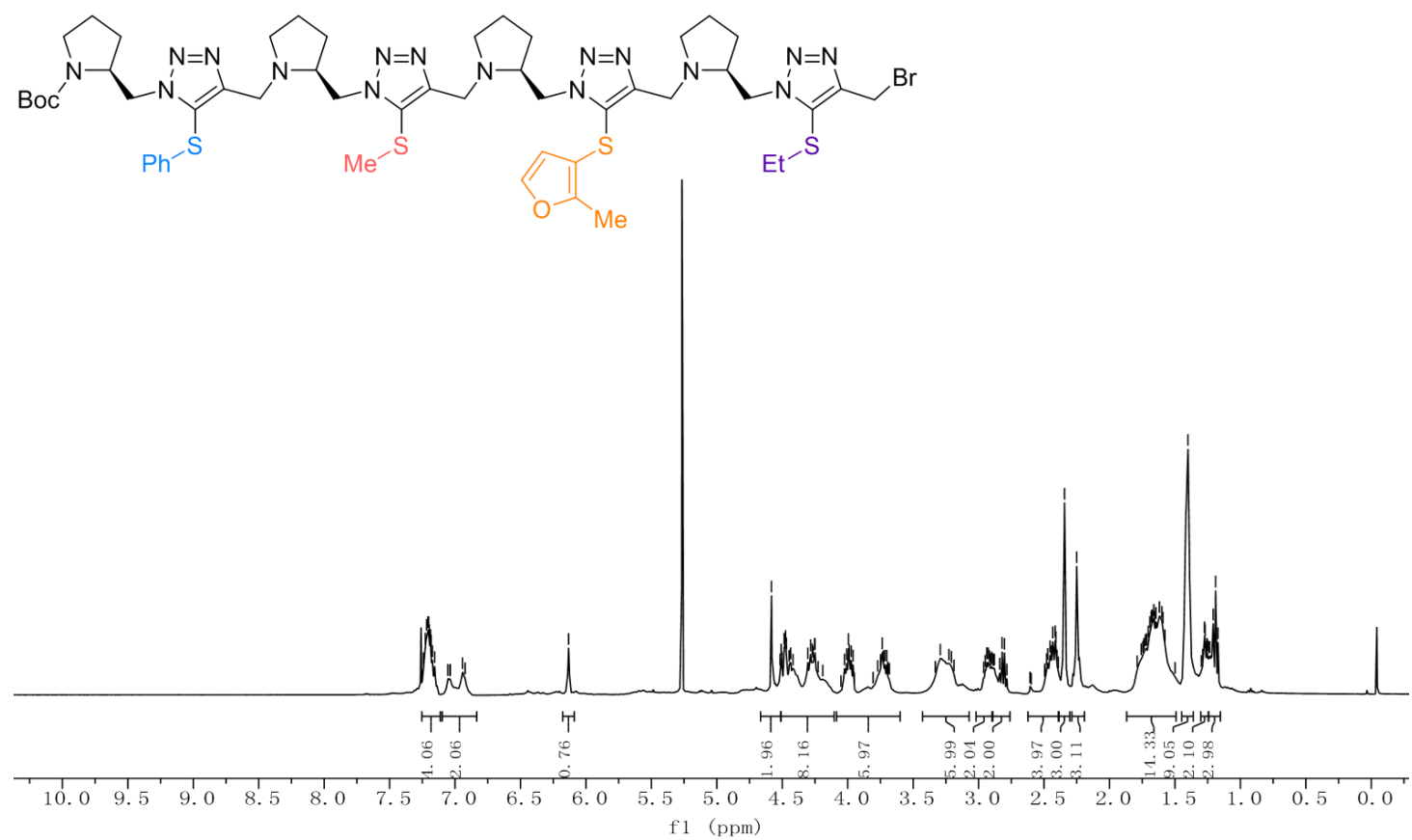

Figure S45. ${ }^{1} \mathrm{H}$ NMR spectra of $\mathbf{6 c - B r}$. 

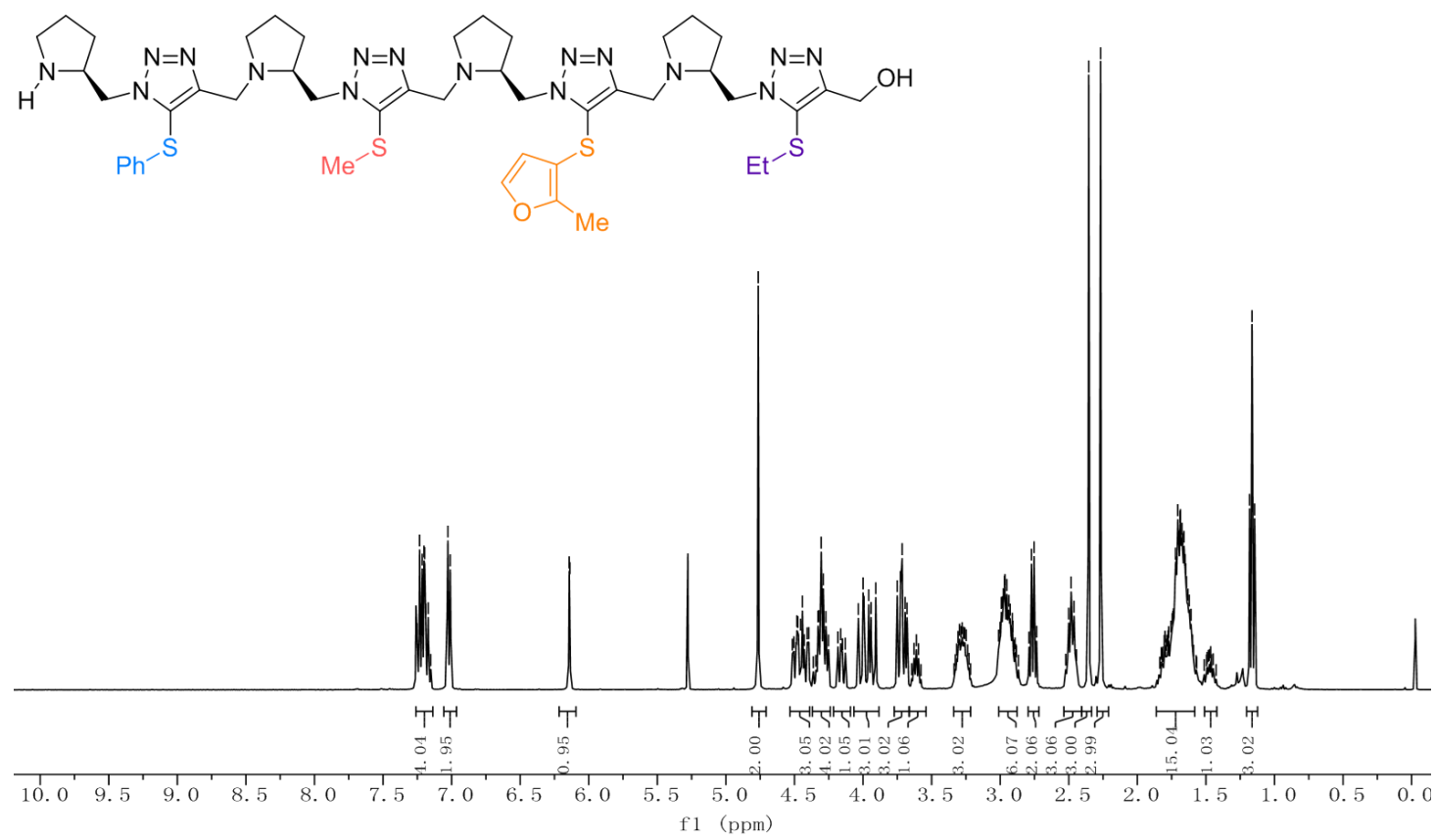

Figure S46. ${ }^{1} \mathrm{H}$ NMR spectra of $6 \mathbf{c - H}$.

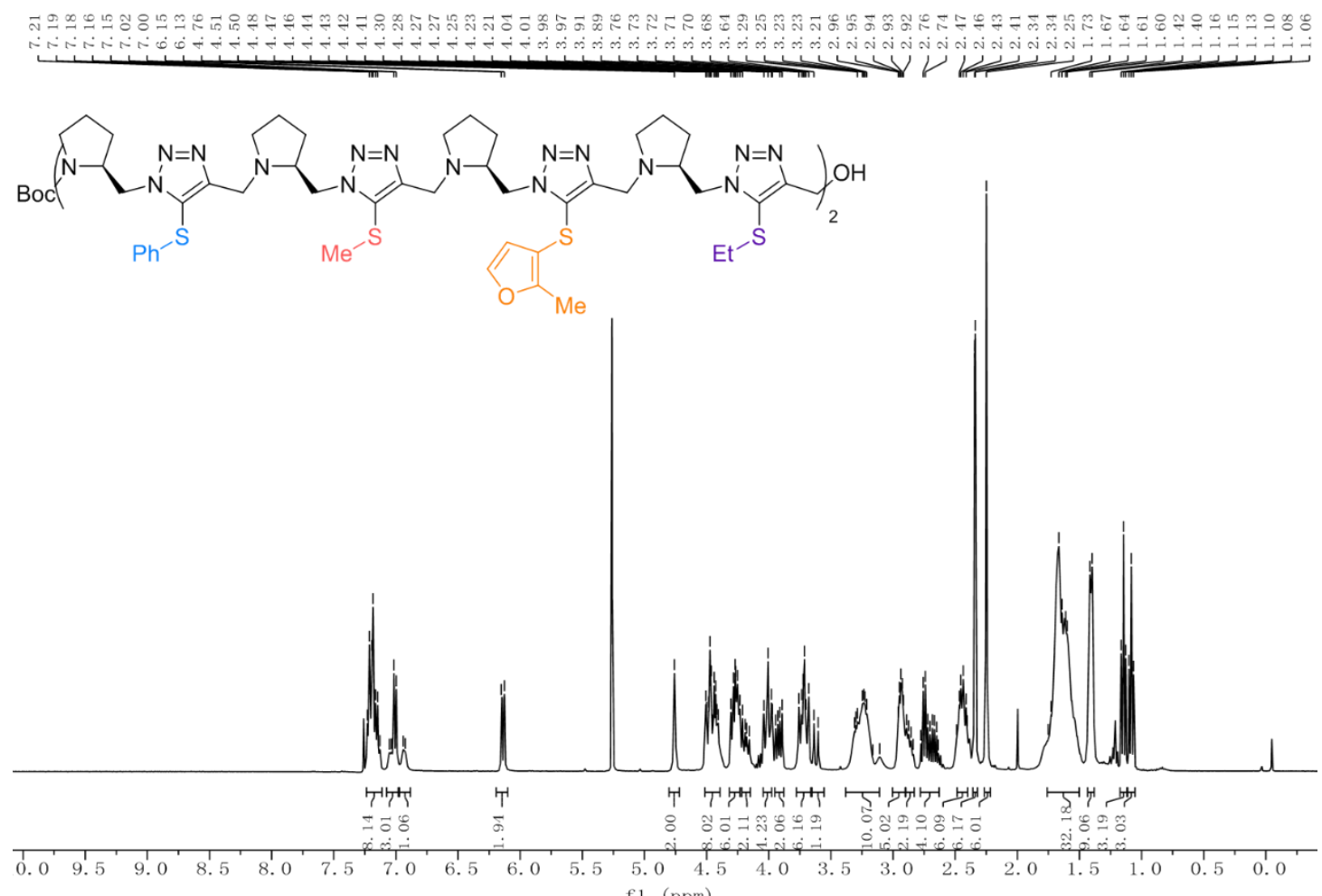

Figure S47. ${ }^{1} \mathrm{H}$ NMR spectra of $8 c$. 


\section{MS Spectra}

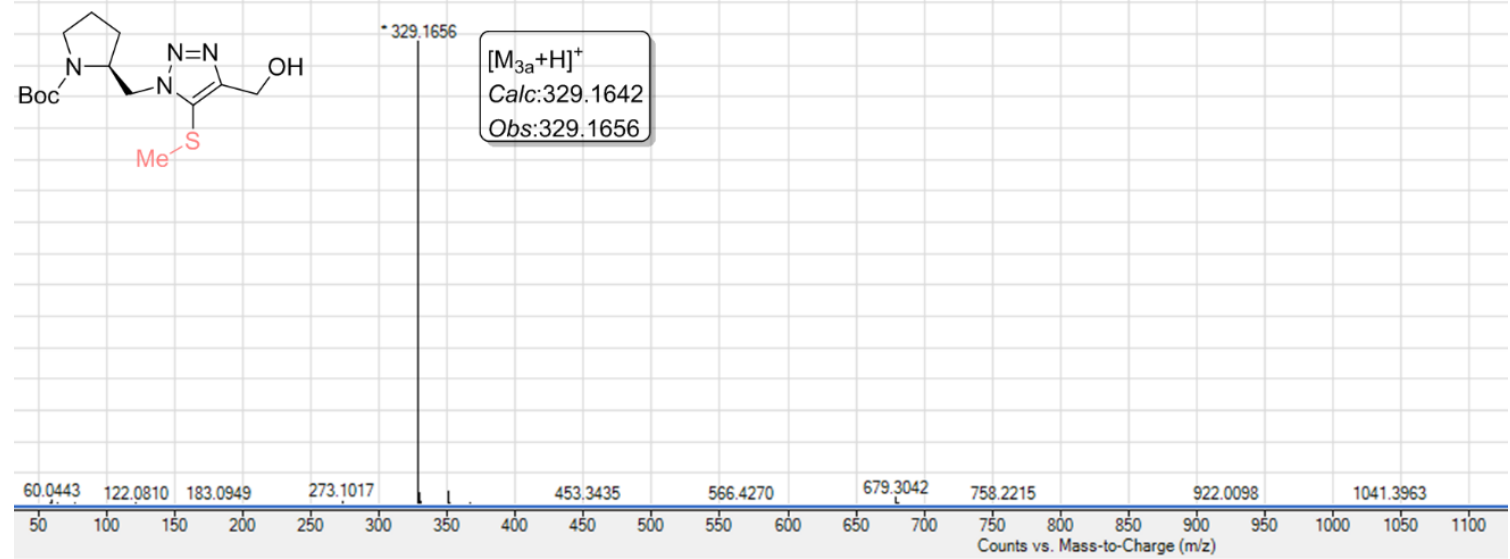

Figure S48. ESI-MS of 3a.

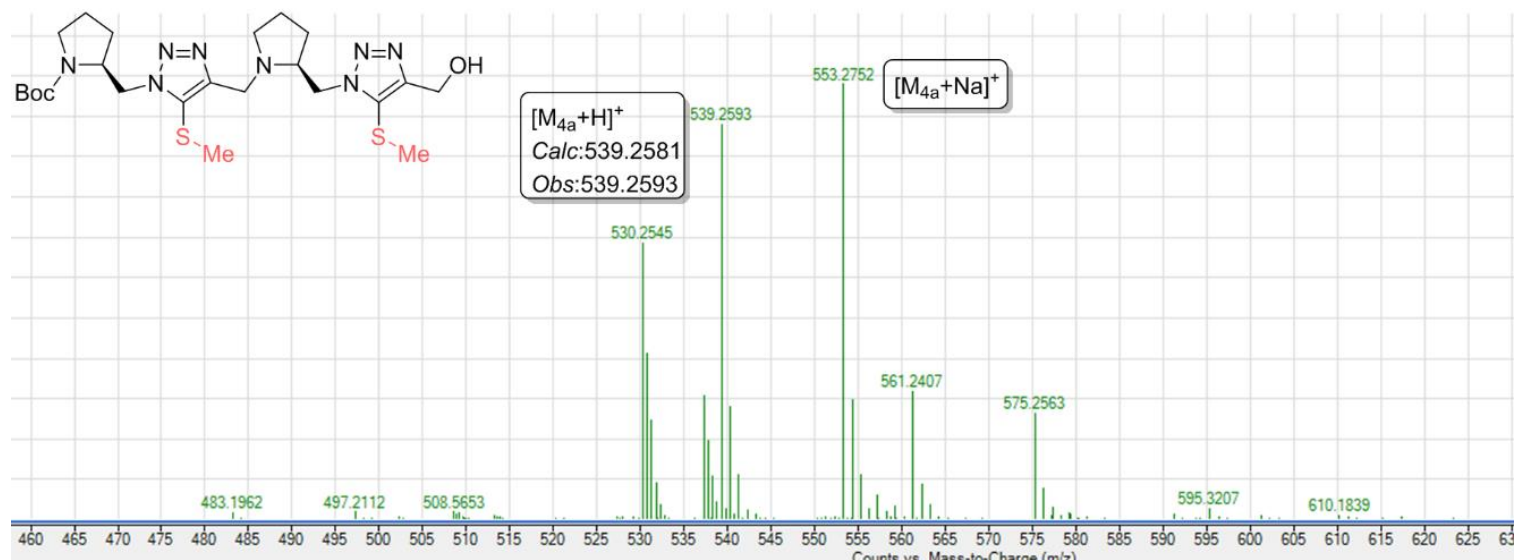

Figure S49. ESI-MS of $4 \mathbf{a}$.

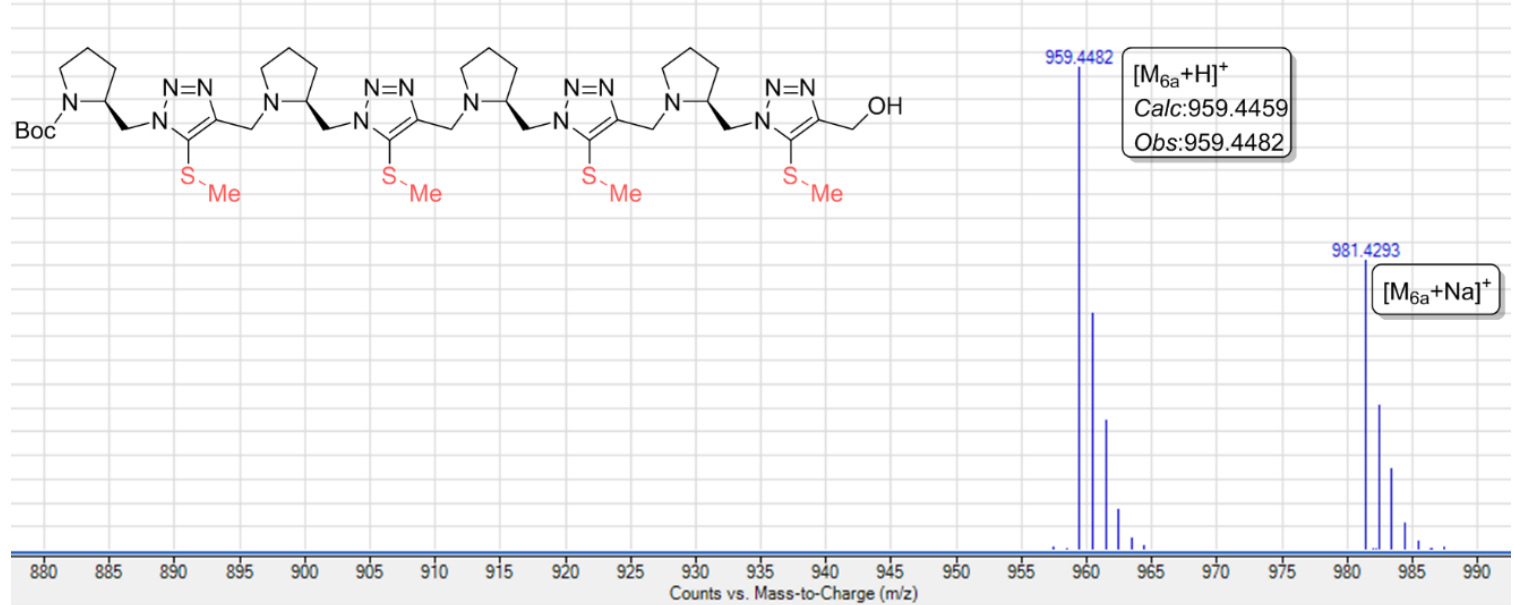

Figure S50. ESI-MS of $6 \mathbf{a}$. 


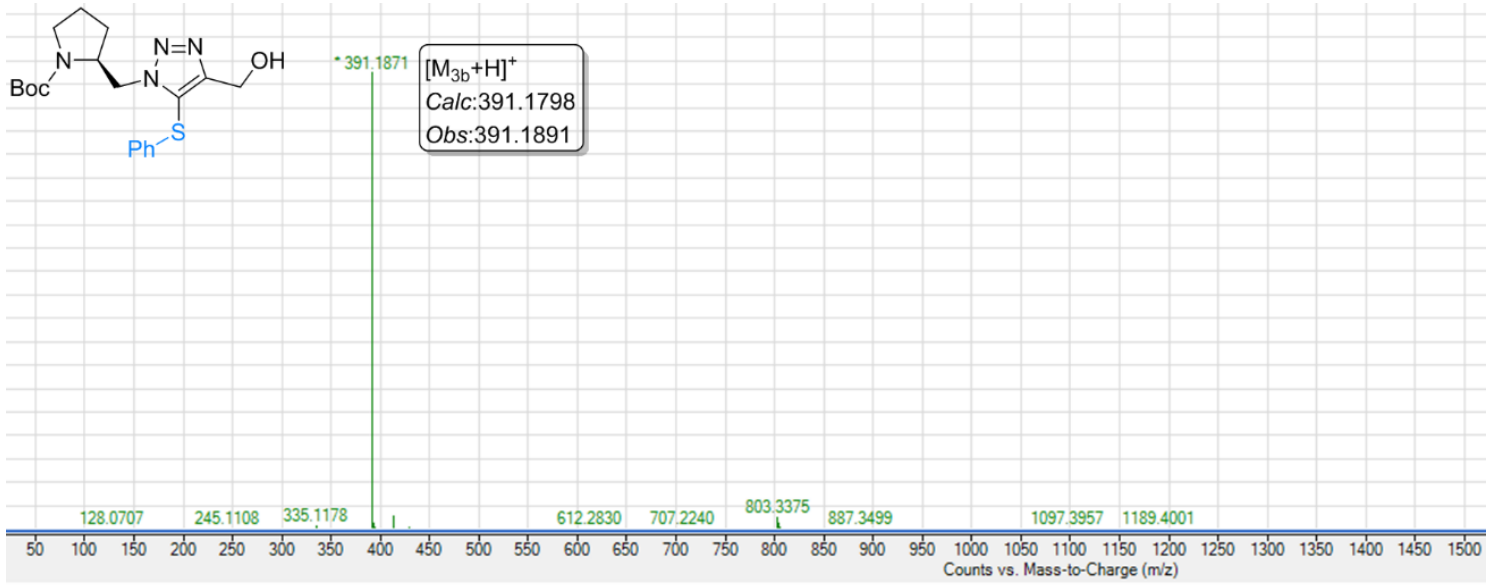

Figure S51. ESI-MS of $\mathbf{3 b}$.

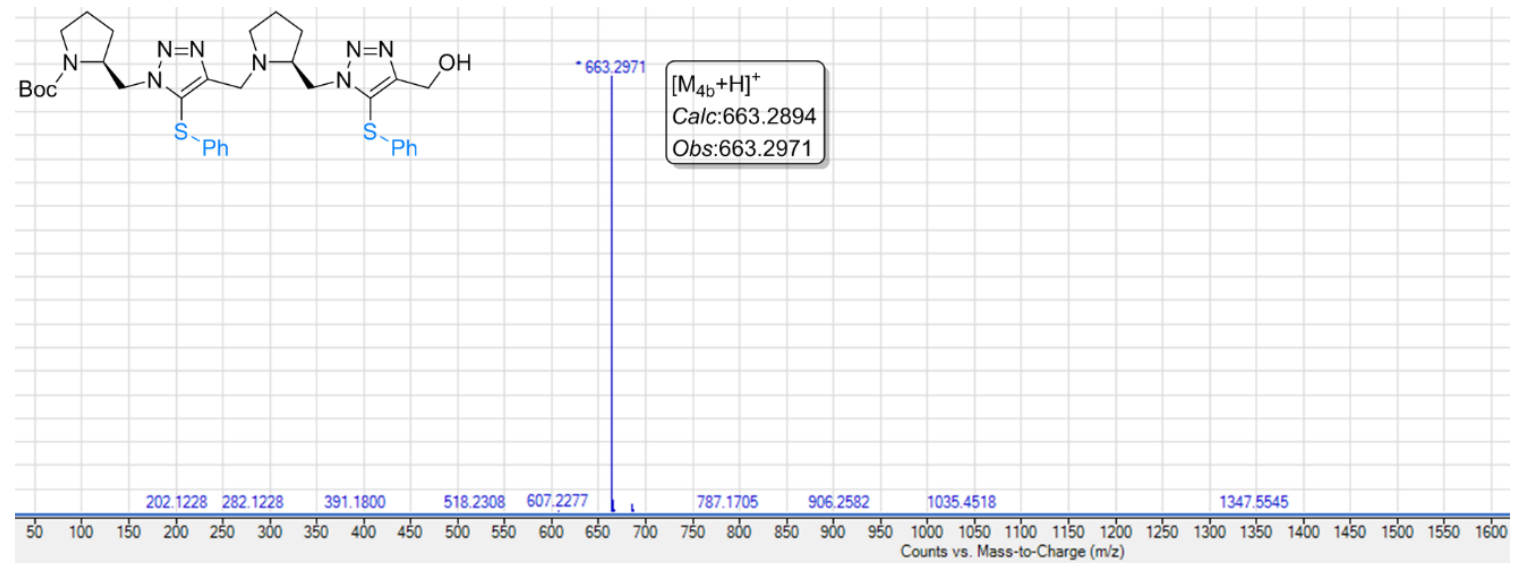

Figure S52. ESI-MS of $\mathbf{4 b}$.

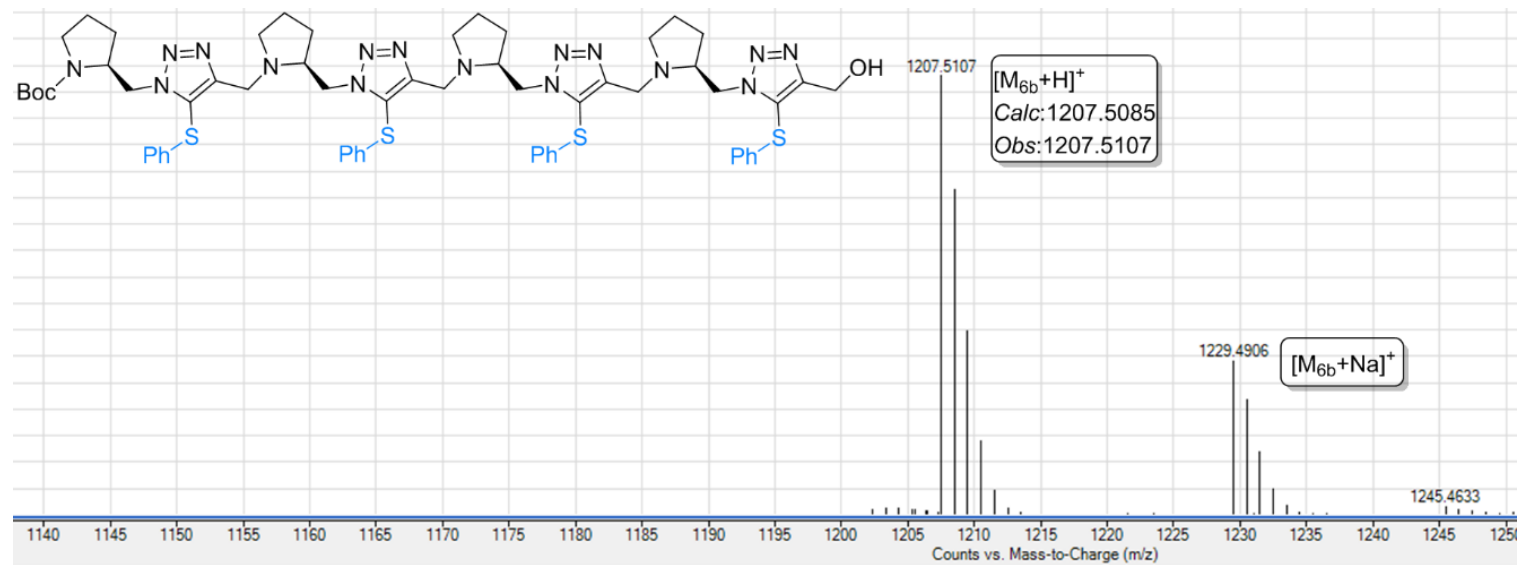

Figure S53. ESI-MS of $\mathbf{6 b}$. 


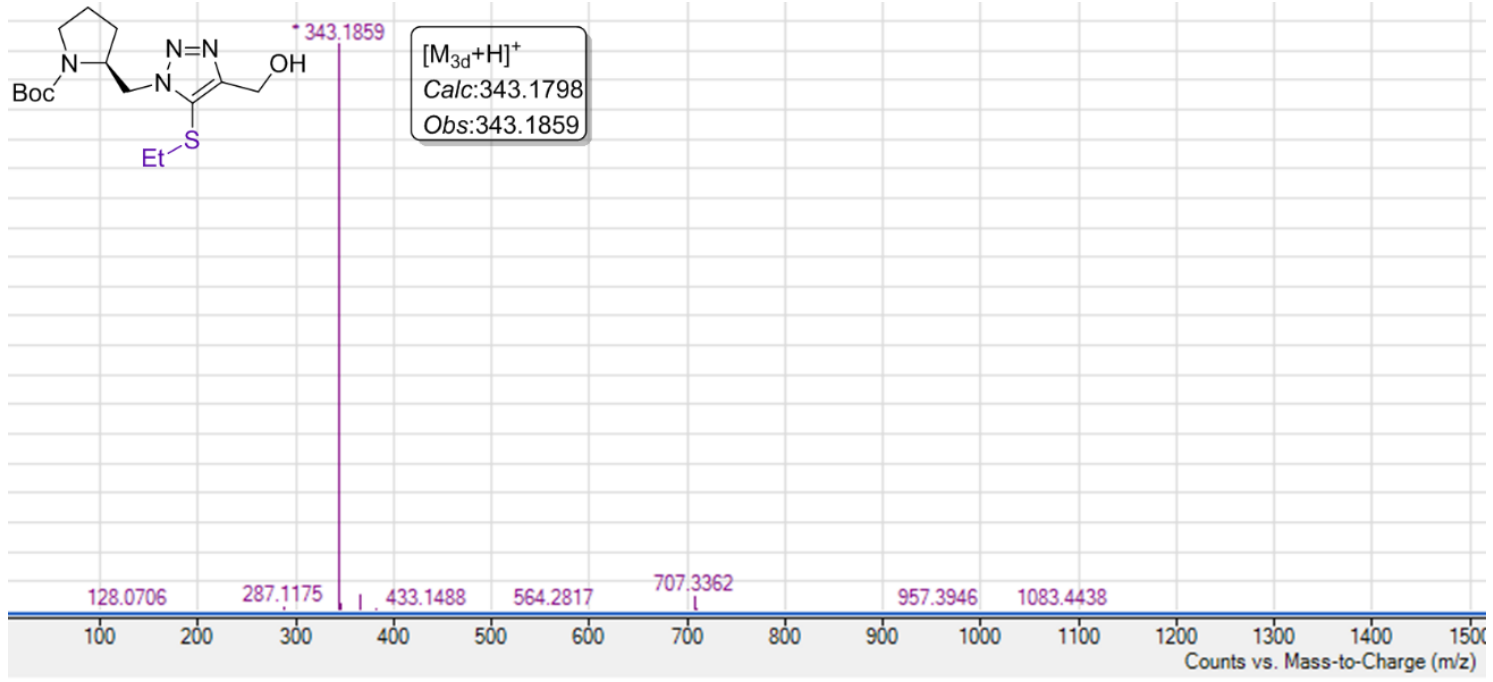

Figure S54. ESI-MS of 3d.

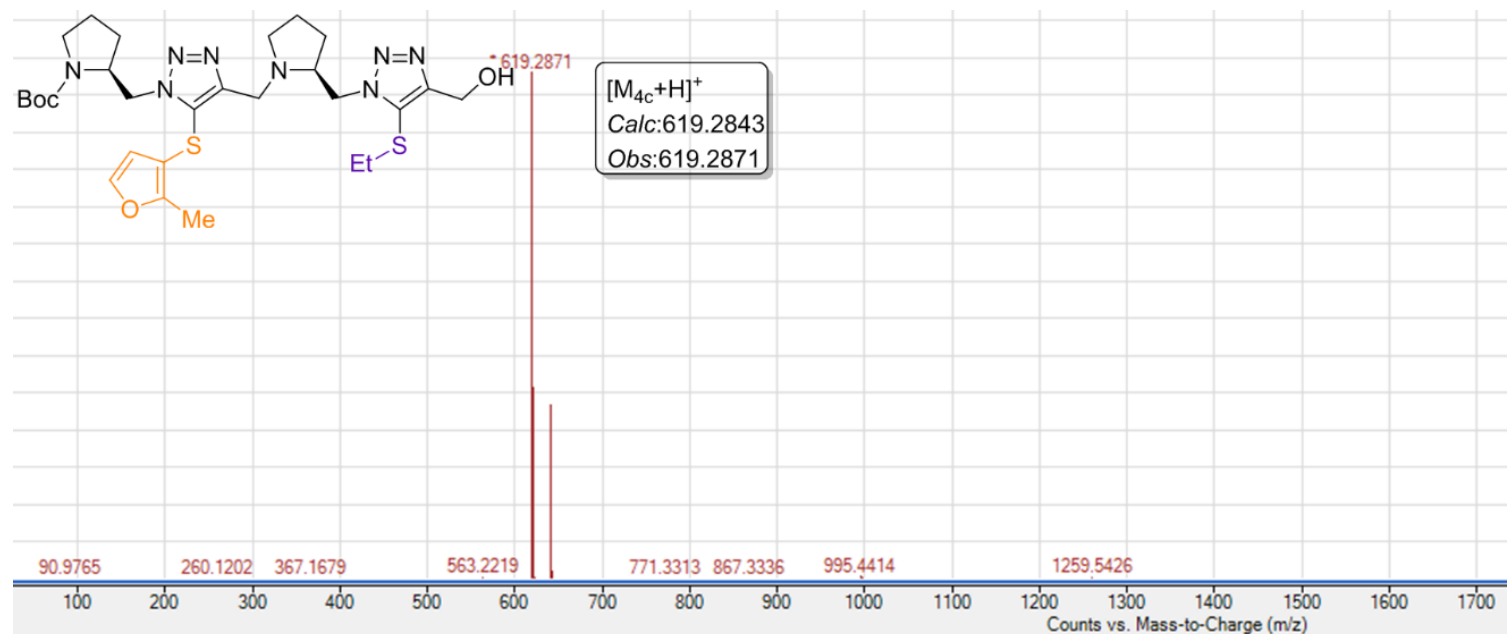

Figure S55. ESI-MS of 4c.

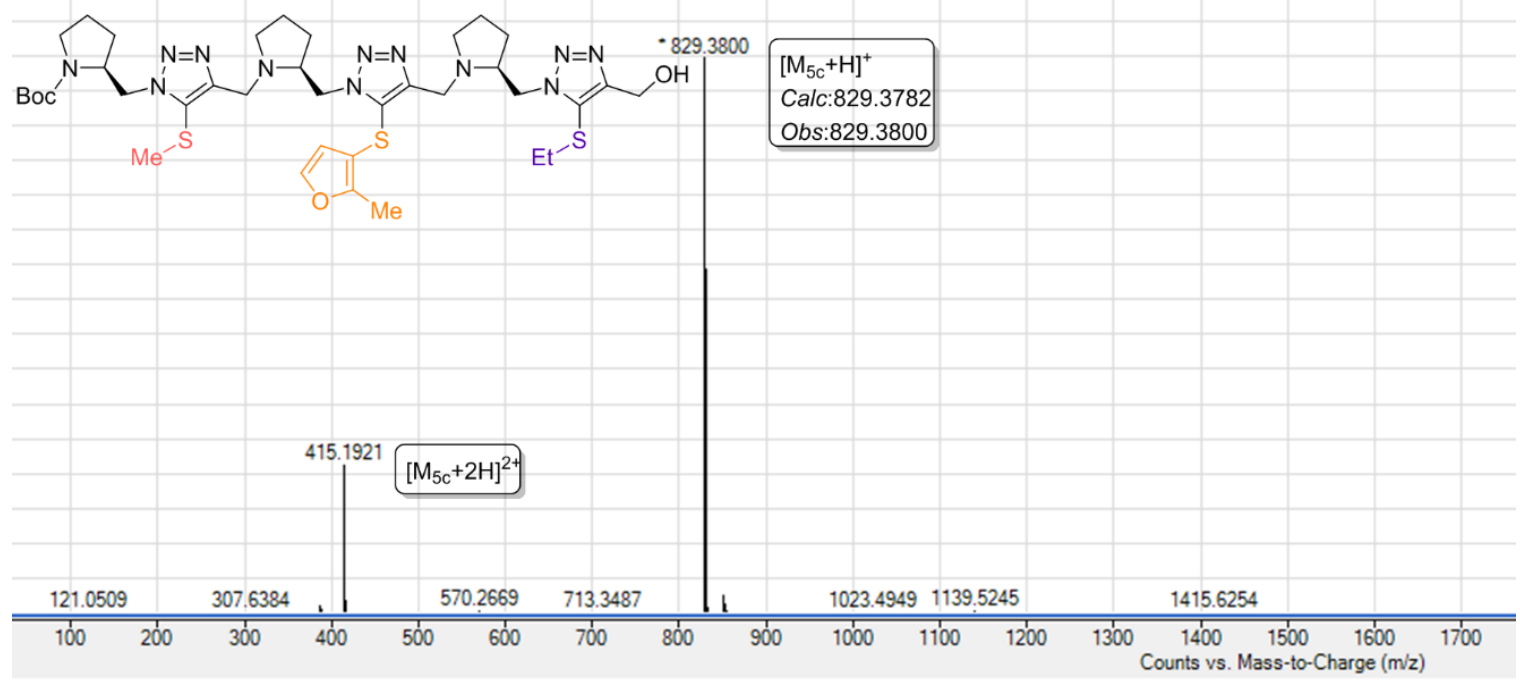

Figure S56. ESI-MS of 5c. 


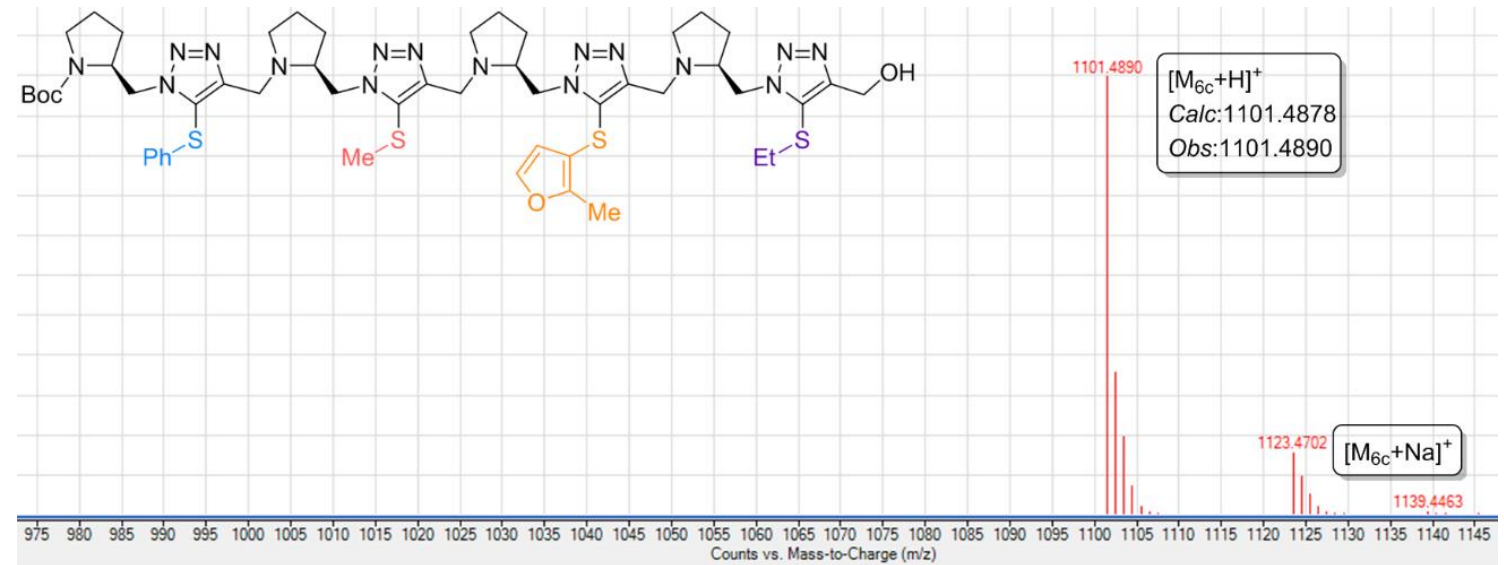

Figure S57. ESI-MS of 6c.

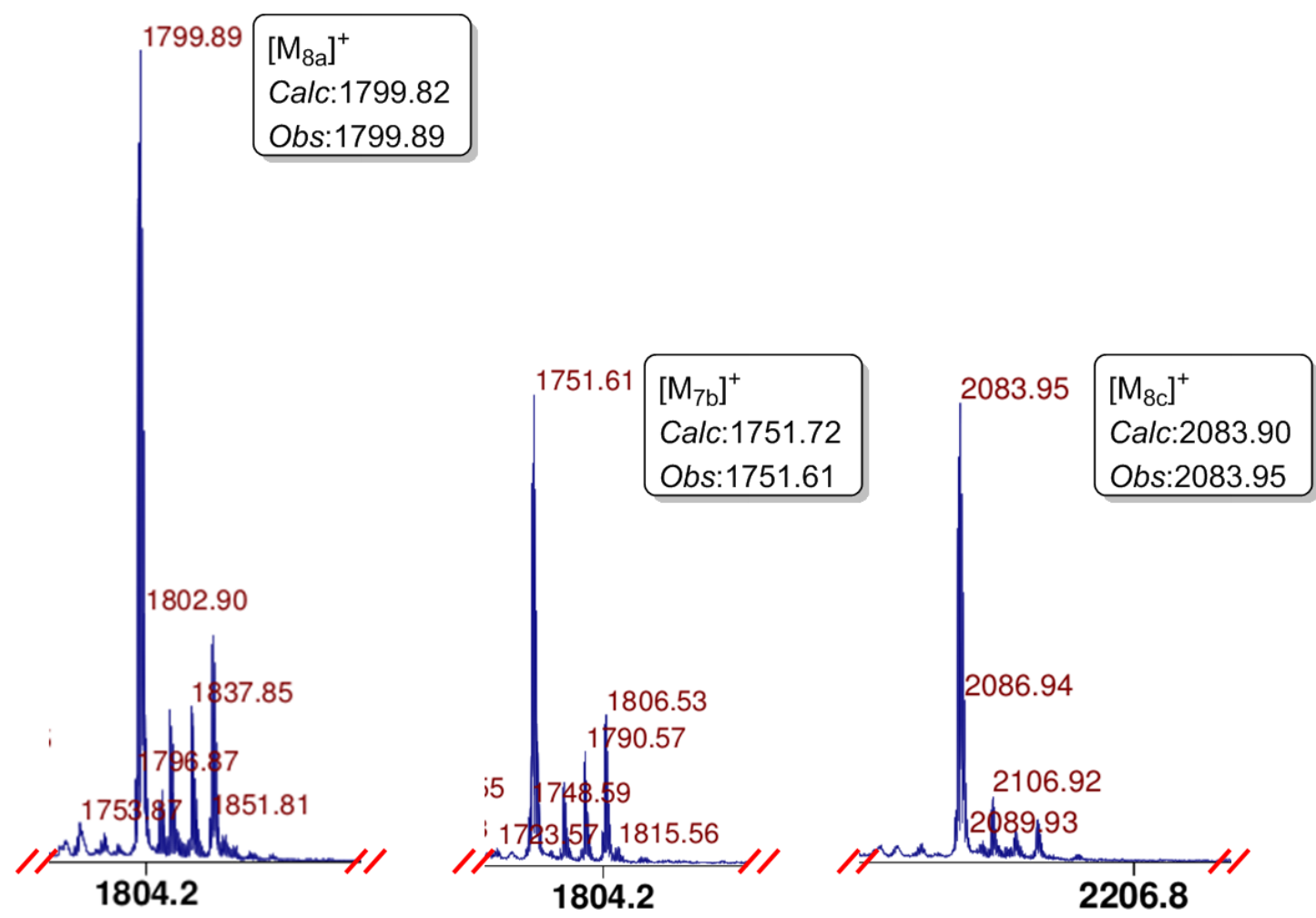

Figure S58. MALDI-TOF-MS of $\mathbf{8 a}, \mathbf{7 b}$ and $\mathbf{8 c}$. 